\title{
Reasons for Dealing with a Particular Bank in the State of Libya
}

\author{
Mohamed Omar Fargani \\ Al-Buraimi University College, Oman
}

\begin{abstract}
This section uses the results of a survey to find out how consumers evaluate banking services in the State of Libya. The survey contained a large number of variables, most of which are correlated. This paper tries to examine the relationships among the interrelated variables and represent them in terms of a few underlying factors. This is done through the use of the technique of Factor Analysis. This study represents an inquiry into consumers' evaluation of the banking system in Libya. A survey was conducted to collect information on a number of issues relating to the consumer's views on various aspects of the structure and performance of commercial banks operating in the State of Libya. The survey results were subjected to multivariate statistical analysis. The technique of factor analysis was used to analyze the relationships among the interrelated variables affecting consumers' choice of a particular commercial bank. The principal component method, with varimax rotation, is utilized to reduce the large number of explanatory variables to a few underlying factors.
\end{abstract}

Keywords: State of Libya, commercial bank, Factor Analysis, varimax rotation

\section{Introduction}

Libya, like many other Middle Eastern countries, has a dual banking system, i.e., interest-free financial organizations operating side-by-side with traditional (interest-based) banks. Libya has 14 banks: eight local conventional banks, one Islamic bank (i.e., operating according to Islamic laws which prohibit the payment of fixed interest on deposits and the charge of fixed interest on loans), and five branches of foreign conventional banks. The study is based on a number of hypotheses including:

1. The Libyan consumers are free to deal with any one (or more) of the commercial banks operating in the State.

2. The customers are aware of the services and terms of the various financial institutions operating in Libyan money market.

The type of services and modes of operations differ, to some extent, between different banks operating in Libya.

The paper is divided into six sections. Following the introduction, Section Two gives information about the used sample. Section Three briefly reviews the technique of factor analysis. Section Four gives reasons for dealing with a particular bank. The main statistical results are shown in Section Five. Finally, Section Six summarizes the main conclusions of the study.

Mohamed Omar Fargani, associate professor, Department of Business Administration and Accounting, Al-Buraimi University College, Oman. 


\section{The Sample}

A survey was conducted during the months of June to August 2017 to find out how the consumers evaluate banking services in the State of Libya. A random sample, with 385 members, was selected.

The sample size was determined using the following assumptions:

1. A proportion $(\pi)$ of $50 \%$. This is the safest possible assumption.

2. A confidence level of $95 \%$. This corresponds to a $Z$ value of 1.96 .

3. A precision rate of $0.05 \%$ within the confidence level.

Under these assumptions, the sample size will be given by:

$$
\begin{gathered}
n=\frac{Z^{2} p(1-p)}{e^{2}} \\
n=\frac{(1.96)^{2}(.5)(.5)}{(0.05)^{2}} \\
\approx 385
\end{gathered}
$$

Hence, a sample size of 385 members was determined.

\section{Methodology}

Factor Analysis is unduly used by social scientists as a variable-reducing technique. This section briefly reviews the mathematics of this technique. The review is heavily based on the work developed by Muliak (1972) and summarized by Malhotra (2004).

Mathematically, Factor Analysis is somewhat similar to multiple regression analysis, in that each variable is expressed as a linear combination of underlying factors. The amount of variance one variable shares with all other variables included in the analysis is referred to as communality (Dillon \& Goldstein, 1984; Bartholomew \& Knott, 1999). The co-variation among the variables is described in terms of a small number of common factors plus a unique factor for each variable (Joreskog \& Sorborn, 1979). These factors are not overtly observed. If the variables are standardized, the factor model may be represented as:

$$
X_{i}=A_{i 1} F_{1}+A_{i 2} F_{2}+A_{i 3} F_{3}+\ldots+A_{i m} F_{m}+V_{i} U_{i}
$$

where:

$X_{I}=i$ th standardized variable;

$A_{i j}=$ standardized multiple regression coefficient of variable $i$ on common factor $j$;

$F_{i}=$ common factor;

$V_{i}=$ standardized regression coefficient of variable i on unique factor $i$;

$U_{I}=$ the unique factor of variable $i$;

$M=$ number of common factors.

The unique factors are non-correlated with each other and with the common factors (The common factors themselves can be expressed as linear combinations of the observed variables).

$$
F_{i}=W_{i 1} X_{1}+W_{i 2} X_{2}+W_{i 3} X_{3}+\ldots+W_{i k} X_{k}
$$

where:

$F=$ estimate of its factor; 
$W_{i}=$ weight or factor score coefficient;

$k=$ number of variables.

It is possible to select weights or factor score coefficients so that the first factor explains the largest portion of the total variance (Manly, 1994; Lattin, Carroll, \& Green, 2003). Then, a second set of weights can be selected, so that the second factor accounts for most of the residual variance, subject to being non-correlated with the first factor. This same principal could be applied to selecting additional weights for the additional factors (Metwally, 2000). Thus, the factors can be estimated so that their factor scores, unlike the values of the original variables, are not correlated. Furthermore, the first factor accounts for the highest variance in the data, the second factor the second highest, and so on.

In the Factor Analysis Model, hypothetical components are derived that account for the linear relationship between observed variables (Harman, 1967; Ding, 1999). The model requires that the relationships between observed variables be linear and that the variables have non-zero correlation between them. The derived hypothetical components have the following properties:

1. They form a linearly independent set of variables. No hypothetical component is derivable from the other hypothetical components as a linear combination of them.

2. The hypothetical components' variables can be divided into two basic kinds of components: common factors and unique factors. These two components can be distinguished in terms of the patterns of weights in the linear equations that derive the observed variables from the hypothetical components' variables. A common factor has more than one variable with a non-zero weight or factor loading associated with the factor. A unique factor has only one variable with a non-zero weight associated with the factor. Hence, only one variable depends upon a unique factor.

3. Common factors are always assumed to be non-correlated with the unique factors. Unique factors are also usually assumed to be mutually non-correlated, but common factors may or may not be correlated with each other.

4. Generally, it is assumed that there are fewer common factors than observed variables. However, the number of unique factors is usually assumed to be equal to the number of observed variables.

The following notations are used:

$X=A n n \times 1$ random vector of observed random variables $X_{1}, X_{2}, X_{3}, \ldots X_{n}$. It is assumed that $E(X)=0$, and $E\left(X X^{\prime}\right)=\mathrm{R}_{\mathrm{xx}}$, a correlation matrix with units in the main diagonal.

$F=A n m \times 1$ vector of $m$ common factors $F_{1}, F_{2}, \ldots F_{m}$. It is assumed that $E(F)=0$, and $E\left(F F^{\prime}\right)=R_{f f '}$, a correlation matrix.

$U=A n n \times 1$ random vector of the $n$ unique factors variables $U_{1}, U_{2}, \ldots U_{n}$. It is assumed that $E(U)=0$, and $E\left(U U^{\prime}\right)=$ I. The unique factors are normalized to have unit variances and are mutually non-correlated.

$A=A n n \times m$ matrix of coefficients called the factor pattern matrix.

$V=A n n \times n$ diagonal matrix of coefficients for theunique factors.

The observed variables, which are the coordinates of $X$, are weighted combinations of the common factors and the unique factors.

The fundamental equation of factor analysis can then be written as:

$$
X=A F+V U
$$

The correlation coefficients between variables in terms of the factors may be derived as follows: 


$$
\begin{aligned}
R x X & =E\left(X X^{\prime}\right) \\
& =E\left\{(A F+V U)(A F+V U)^{\prime}\right\} \\
& =E\left\{(A F+V U)\left(F^{\prime} A^{\prime}+U^{\prime} V^{\prime}\right)\right\} \\
& =E\left(A F F^{\prime} A^{\prime}+A F U^{\prime} V^{\prime}+V U F^{\prime} A^{\prime}+V U U^{\prime} V^{\prime}\right) \\
& =A R_{f f} A^{\prime}+A R_{f u} V^{\prime}+V R_{u f} A^{\prime}+V^{2}
\end{aligned}
$$

Given that the common factors are non-correlated with the unique factors, we have:

$$
R_{f u}=R_{u f}{ }^{\prime}=0 \text {. }
$$

Hence,

$$
R_{x x}=A R_{f f} A^{\prime}+V^{2}
$$

Suppose we subtract the matrix of unique factor variance $V^{2}$, from both sides. We then obtain:

$$
R_{x x}-V^{2}=A R_{f f} A^{\prime}
$$

$R x x$ is dependent only upon the common factor variables, and the correlation among the variables, are related only to the common factors. Let

$$
R_{c}=R_{x X}-V^{2}
$$

We have already defined the factor pattern matrix $A$. The coefficients of the factor pattern matrix are weights assigned to the common factors when the observed variables are expressed as linear combinations of the common and unique factors. We now define the factor structure matrix. The coefficients of the factor structure matrix are the covariances between the observed variables and the factors. The factor structure matrix is helpful in the interpretation of factors as it shows which variables are similar to a common factor variable (Tacq, 1997; Wichern, 2001). The factor structure matrix $A_{s}$, is defined as:

$$
\begin{aligned}
A_{s} & =E\left(X F^{\prime}\right) \\
& =E\left[(A F+V U) F^{\prime}\right] \\
& =A R_{f f}+V R_{u f} \\
& =A R_{f f}
\end{aligned}
$$

Thus, the factor structure matrix is equivalent to the factor pattern matrix $A$ multiplied by the matrix of covariance's among the factors $R_{f f}$. Substituting $A_{s}$ for $A R_{f f}$, the reduced correlation matrix becomes the product of the factor structure and the factor pattern matrix (Johnson \& Wichern, 2002).

$$
\begin{aligned}
R_{C} & =A R_{f f} A^{\prime} \\
& =A_{s} A^{\prime}
\end{aligned}
$$

\section{Reasons for Dealing With a Particular Bank}

This section uses the results of the survey to find out how consumers evaluate banking services in the State of Libya. The survey contained a large number of variables, most of which are correlated. This section tries to examine the relationships among the interrelated variables and represent them in terms of a few underlying factors. This is done through the use of the technique of Factor Analysis.

Respondents were asked to indicate their degree of agreement with 26 statements relating to their preferences for dealing with a particular bank, using a seven-point scale. The survey results were analyzed using the SPSS program (Coakes \& Steed, 1999). Table 1 gives the means and standard deviations of scores of variables related to consumers' preferences. The data in Table 1 suggest that cost of keeping a current account (V17); Return on deposits (V16), and Staff confidence (V3) score relatively higher than other variables. Other variables that score relatively high are bank appearance (V5); easiness to obtain loans (V2), and bank 
reputation (V4), on the other hand, the mean score of variables 14 and 10, which relate to easiness to deposit and withdraw and speed of services are much smaller than other scores. The standard deviation was highest for variable V18 (easiness to transfer money abroad). Other variables which have relatively high standard deviations are V22 (bank location) and (V1) (number of branches). On the other hand, the variable V14 (easiness to deposit and withdraw) has the lowest standard deviation. Other variables which have relatively low standard deviations are V19 (staff assistance) and V19 (interest on loans).

\section{Statistical Results}

The main results of Factor Analysis are given in Tables 2 to 11. An investigation of the results would seem to suggest that:

1. All coefficients on the diagonals of the Anti-image correlation matrix (see Table 2) are greater than 0.5. Therefore, no variable should be eliminated.

2. The correlation matrix (see Table 3) shows that 250 correlation coefficients out of 300 coefficients (i.e., $83.3 \%$ ) are statistically significant at, at least, the $5 \%$ level of significance.

3. Table 3 also shows that each variables has a highly significant correlation coefficient with more than one of the other variables. This suggests adequacy of the factor mode.

Table 1

Means and Standard Deviations of Scores of Variables Related to Bank Services in Libya Descriptive Statistics

\begin{tabular}{|c|c|c|c|c|}
\hline & Mean & Std. Deviation & Analysis N & \\
\hline V1 & 4.1013 & 1.7283 & 385 & Number of branches \\
\hline $\mathrm{V} 2$ & 4.6519 & 1.7893 & 385 & Easiness to obtain loans \\
\hline V3 & 3.7351 & 1.5720 & 385 & Staff competence \\
\hline V4 & 4.6519 & 1.3163 & 385 & Bank reputation \\
\hline V5 & 4.6649 & 1.3266 & 385 & Bank appearance \\
\hline V6 & 4.5039 & 1.3270 & 385 & Community service \\
\hline V7 & 4.2494 & 1.3675 & 385 & The bank is known abroad \\
\hline V8 & 5.0519 & 1.3258 & 385 & Size of the bank \\
\hline V9 & 4.8727 & 1.2837 & 385 & Interest on loans \\
\hline V10 & 3.2260 & 1.4942 & 385 & Speed of services \\
\hline V11 & 4.3221 & 1.3249 & 385 & Advertising \\
\hline V12 & 3.3039 & 1.4374 & 385 & Banking facilities \\
\hline V13 & 3.3662 & 1.4337 & 385 & Easiness to open a current account \\
\hline V14 & 3.1766 & 1.2353 & 385 & Easiness to deposit and withdraw \\
\hline V15 & 4.2338 & 1.3683 & 385 & Relatives and friends recommendations \\
\hline V16 & 5.1636 & 1.2917 & 385 & Return on deposits \\
\hline V17 & 5.1740 & 1.2741 & 385 & Cost of keeping a current account \\
\hline V18 & 3.6234 & 1.8543 & 385 & Easiness to transfer money abroad \\
\hline V19 & 3.3429 & 1.2673 & 385 & Staff assistance \\
\hline V20 & 3.9169 & 1.5707 & 385 & Religious reasons \\
\hline $\mathrm{V} 21$ & 3.6208 & 1.5075 & 385 & Afternoon service \\
\hline V22 & 4.1558 & 1.7623 & 385 & Bank location \\
\hline V23 & 3.9039 & 1.6484 & 385 & Variety of services \\
\hline V24 & 3.9662 & 1.6302 & 385 & Easiness to read bank statements \\
\hline $\mathrm{V} 25$ & 3.9065 & 1.6062 & 385 & Available accounts \\
\hline V26 & 3.9662 & 1.6635 & 385 & Electronic services \\
\hline
\end{tabular}


4. Bartlett's test of sphericity was used to test the null hypothesis that the variables are non-correlated in the population. The test gave a value of 16154.9 which is highly significant favoring a rejection of the null hypothesis (Goldstein, 1984; Dunteman, 1989). Also, the Kaiser-Meyer-Olkin (KMO) measure of sampling adequacy was calculated. A value of 0.896 was obtained which indicates that correlations between pairs of variables can be explained by other variables and hence Factor Analysis is appropriate (Hair, Anderson, Joseph, Tatham, \& Blach, 2004). Actually, a KMO value of 0.896 is considered "marvelous".

5. The variance statistics (see Table 7) suggest that there are only five factors with an eigenvalue greater than one. The five factors account for $83.3 \%$. This is a high percentage which suggests adequacy of the factor model.

6. The factor loadings (see Table 8) give the relationship between variables and factors. For example, speed of services (V10) rating can be expressed as:

$$
\mathrm{V} 10=0.788 F_{1}+0.315 F_{2}-0.372 F_{3}+0.123 F_{4}-0.107 F_{5}
$$

Advertising (V11) rating can be expressed as:

$$
\mathrm{V} 11=0.319 F_{1}+0.785 F_{2}+0.269 F_{3}-0.9626 F_{4}-0.189 F_{5}
$$

7. The upper right triangle in the reproduced correlation matrix represents the residuals, i.e., the difference between the observed correlation coefficient and that estimated from the model. The magnitudes of the residuals indicate how well the fitted model reproduces the observed correlations (Morrison, 2005). The results reveal that only $17 \%$ of the residuals are greater than 0.05 (in absolute value). This suggests goodness of fit.

Although the initial or non-rotated factor matrix indicates the relationship between the factors and individual variables, it does not result in factors which can be interpreted (Myers \& Mullet, 2003). For example, Factor 1 in Table 8 is highly correlated with 15 out of the 26 variables. In such a complex matrix, it is difficult to interpret the factors. Therefore, through rotation, the factor matrix is transformed into a simpler one that is easier to interpret.

The rotated factors matrix obtained by the varimax procedure (see Table 10) suggests that:

Factor 1 has high coefficients for the variables which represent:

V1: Number of branches;

V2: Easiness to obtain loans;

V21: Afternoon service;

V22: Bank location;

V23: Variety of services;

V24: Easiness to read bank statements;

V25: Available accounts;

V26: Electronic services.

Therefore, this factor may be labeled "convenience".

Factor 2 has high coefficients for the variables which represent:

V3: Staff competence;

V10: Speed of services;

V12: Banking facilities;

V13: Easiness to open a current account;

V14: Easiness to deposit and withdraw;

V18: Easiness to transfer money abroad; 
V19: Staff assistance.

Therefore, this factor may be labeled "traditional services".

Factor 3 has high coefficients for the variables which represent:

V4: Bank reputation;

V5: Bank appearance;

V6: Community service;

V7: The bank is known abroad;

V8: Size of the bank;

V11: Advertising;

V15: Relatives and friends recommendations.

Therefore, this factor may be labeled "reputation".

Factor 4 has high coefficients for the variables which represent:

V9: Interest on loans;

V16: Return on deposits;

V17: Cost of keeping a current account.

Therefore, this factor may be labeled "cost-return".

Finally, Factor 5 is highly related to the variables representing religion (V20). Hence, this factor may be labeled "religion".

Thus, using the principal component method and varimax rotation, the 26 explanatory variables for dealing with a particular bank, have been reduced to the following five factors:

F1: Convenience;

F2: Traditional services;

F3: Reputation;

F4: Cost-return;

F5: Religion.

\section{Results of Factor Analysis}


Table 2

Anti-image Matrices

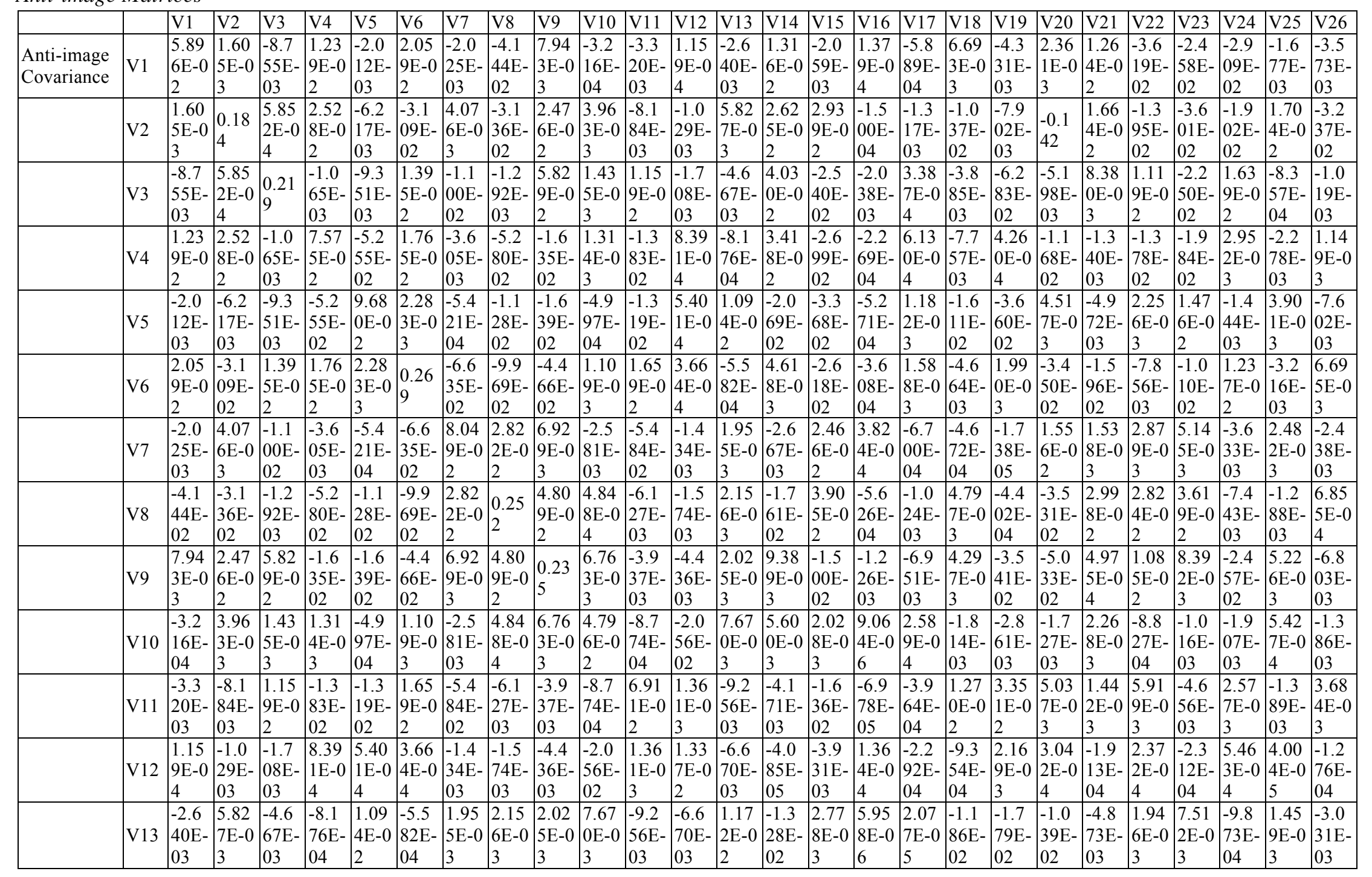


(Table 2 continued)

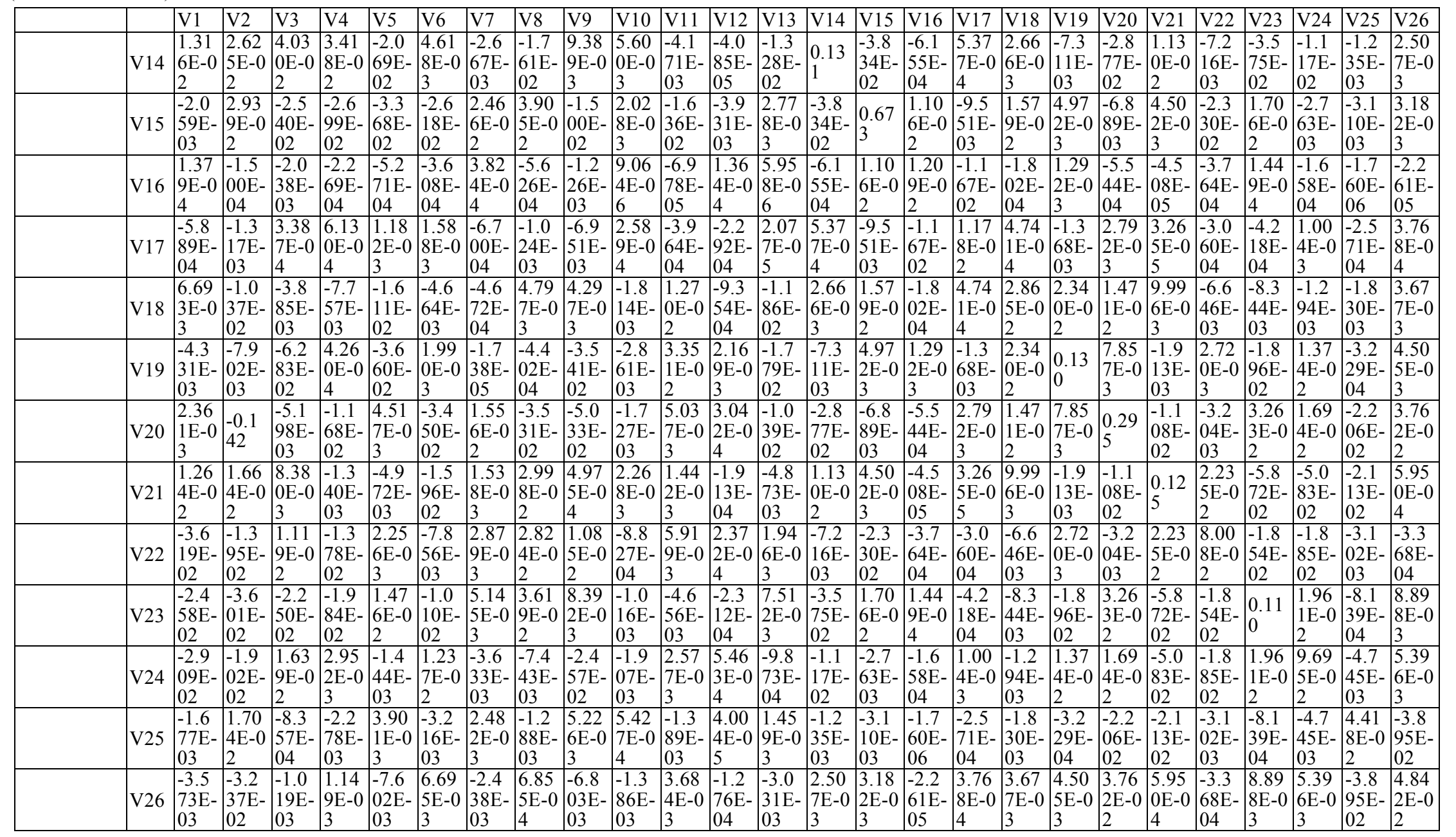


(Table 2 continued)

\begin{tabular}{|c|c|c|c|c|c|c|c|c|c|c|c|c|c|c|c|c|c|c|c|c|c|c|c|c|c|c|c|}
\hline & & $\mathrm{V} 1$ & V2 & V3 & V4 & V5 & V6 & V7 & V8 & V9 & V10 & V11 & V12 & V13 & V14 & V15 & V16 & V17 & V18 & V19 & V20 & $\mathrm{V} 21$ & V22 & V23 & V24 & V25 & V26 \\
\hline \multirow[t]{13}{*}{$\begin{array}{l}\text { Anti-image } \\
\text { Correlation }\end{array}$} & V1 & $\begin{array}{l}0.88 \\
3\end{array}$ & $\begin{array}{l}1.54 \\
2 \mathrm{E}-0 \\
2\end{array}$ & $\begin{array}{l}-7.7 \\
03 \text { E- } \\
02\end{array}$ & $\begin{array}{l}0.18 \\
5\end{array}$ & $\begin{array}{l}-2.6 \\
64 \mathrm{E}- \\
02\end{array}$ & $\begin{array}{l}0.16 \\
4\end{array}$ & $\begin{array}{l}-2.9 \\
39 \mathrm{E}- \\
02\end{array}$ & $\begin{array}{l}-0.3 \\
40\end{array}$ & $\begin{array}{l}6.74 \\
3 \mathrm{E}-0 \\
2\end{array}$ & $\begin{array}{l}-6.0 \\
47 \mathrm{E}- \\
03\end{array}$ & $\begin{array}{l}-5.2 \\
02 \mathrm{E}- \\
02\end{array}$ & $\begin{array}{l}4.12 \\
9 \mathrm{E}-0 \\
3\end{array}$ & $\begin{array}{l}-0.1 \\
00\end{array}$ & $\begin{array}{l}0.15 \\
0\end{array}$ & $\begin{array}{l}-1.0 \\
34 \mathrm{E}- \\
02\end{array}$ & $\begin{array}{l}5.16 \\
4 \mathrm{E}-0 \\
3\end{array}$ & $\begin{array}{l}-2.2 \\
35 \mathrm{E}- \\
02\end{array}$ & $\begin{array}{l}0.16 \\
3\end{array}$ & $\begin{array}{l}-4.9 \\
45 \mathrm{E}- \\
02\end{array}$ & $\begin{array}{l}1.79 \\
0 \mathrm{E}-0 \\
2\end{array}$ & $\begin{array}{l}0.14 \\
7\end{array}$ & $\begin{array}{l}-0.5 \\
27\end{array}$ & \begin{tabular}{|l|}
-0.3 \\
06
\end{tabular} & $\begin{array}{l}-0.3 \\
85\end{array}$ & $\begin{array}{l}-3.2 \\
85 \mathrm{E}- \\
02\end{array}$ & $\begin{array}{l}-6.6 \\
88 \mathrm{E}- \\
02\end{array}$ \\
\hline & V2 & $\begin{array}{l}1.54 \\
2 \mathrm{E}-0 \\
2\end{array}$ & $\begin{array}{l}0.84 \\
3\end{array}$ & $\begin{array}{l}2.91 \\
7 \mathrm{E}-0 \\
3\end{array}$ & $\begin{array}{l}0.21 \\
4\end{array}$ & $\begin{array}{l}-4.6 \\
62 \mathrm{E}- \\
02\end{array}$ & $\begin{array}{l}-0.1 \\
40\end{array}$ & $\begin{array}{l}3.35 \\
2 \mathrm{E}-0 \\
2\end{array}$ & $\begin{array}{l}-0.1 \\
46\end{array}$ & $\begin{array}{l}0.11 \\
9\end{array}$ & $\begin{array}{l}4.22 \\
2 \mathrm{E}-0 \\
2\end{array}$ & $\begin{array}{l}-7.2 \\
63 \mathrm{E}- \\
02\end{array}$ & $\begin{array}{l}-2.0 \\
77 \mathrm{E}- \\
02\end{array}$ & $\begin{array}{l}0.12 \\
6\end{array}$ & $\begin{array}{l}0.16 \\
9\end{array}$ & $\begin{array}{l}8.36 \\
0 \mathrm{E}-0 \\
2\end{array}$ & \begin{tabular}{|l}
-3.1 \\
$83 \mathrm{E}-$ \\
03
\end{tabular} & \begin{tabular}{|l}
-2.8 \\
$31 \mathrm{E}-$ \\
02
\end{tabular} & $\begin{array}{l}-0.1 \\
43\end{array}$ & \begin{tabular}{|l}
-5.1 \\
$11 \mathrm{E}-$ \\
02
\end{tabular} & $\begin{array}{l}-0.6 \\
12\end{array}$ & $\begin{array}{l}0.11 \\
0\end{array}$ & $\begin{array}{l}-0.1 \\
15\end{array}$ & $\begin{array}{l}-0.2 \\
54\end{array}$ & $\begin{array}{l}-0.1 \\
43\end{array}$ & $\begin{array}{l}0.18 \\
9\end{array}$ & \begin{tabular}{|l}
-0.3 \\
43
\end{tabular} \\
\hline & V3 & \begin{tabular}{|l}
-7.7 \\
$03 \mathrm{E}-$ \\
02 \\
\end{tabular} & $\begin{array}{l}2.91 \\
7 \mathrm{E}-0 \\
3\end{array}$ & $\begin{array}{l}0.93 \\
5\end{array}$ & \begin{tabular}{|l}
-8.2 \\
$68 \mathrm{E}-$ \\
03 \\
\end{tabular} & \begin{tabular}{|l}
-6.4 \\
$21 \mathrm{E}-$ \\
02
\end{tabular} & $\begin{array}{l}5.74 \\
7 \mathrm{E}-0 \\
2\end{array}$ & \begin{tabular}{|l}
-8.2 \\
$81 \mathrm{E}-$ \\
02
\end{tabular} & $\begin{array}{l}-5.5 \\
03 \mathrm{E}- \\
03\end{array}$ & $\begin{array}{l}0.25 \\
7\end{array}$ & $\begin{array}{l}1.40 \\
0 \mathrm{E}-0 \\
2\end{array}$ & $\begin{array}{l}9.42 \\
0 \mathrm{E}-0 \\
2\end{array}$ & $\begin{array}{l}-3.1 \\
56 \mathrm{E}- \\
02\end{array}$ & \begin{tabular}{|l}
-9.2 \\
$10 \mathrm{E}-$ \\
02
\end{tabular} & $\begin{array}{l}0.23 \\
8\end{array}$ & \begin{tabular}{|l}
-6.6 \\
$14 \mathrm{E}-$ \\
02 \\
\end{tabular} & \begin{tabular}{|l}
3.9 \\
$60 \mathrm{E}-$ \\
02
\end{tabular} & \begin{tabular}{|l|}
6.66 \\
$8 \mathrm{E}-0$ \\
3 \\
\end{tabular} & \begin{tabular}{|l}
-4.9 \\
$04 \mathrm{E}-$ \\
02
\end{tabular} & $\begin{array}{l}-0.3 \\
72\end{array}$ & \begin{tabular}{|l}
-2.0 \\
$44 \mathrm{E}-$ \\
02
\end{tabular} & $\begin{array}{l}5.05 \\
8 \mathrm{E}-0 \\
2 \\
\end{array}$ & $\begin{array}{l}8.45 \\
1 \mathrm{E}-0 \\
2\end{array}$ & $\begin{array}{l}-0.1 \\
45\end{array}$ & $\begin{array}{l}0.11 \\
2\end{array}$ & \begin{tabular}{|l}
-8.4 \\
$93 \mathrm{E}-$ \\
03
\end{tabular} & \begin{tabular}{|l}
-9.8 \\
$89 \mathrm{E}-$ \\
03 \\
\end{tabular} \\
\hline & V4 & $\begin{array}{l}0.18 \\
5\end{array}$ & $\begin{array}{l}0.21 \\
4\end{array}$ & \begin{tabular}{|l}
-8.2 \\
$68 \mathrm{E}-$ \\
03
\end{tabular} & $\begin{array}{l}0.81 \\
1\end{array}$ & $\begin{array}{l}-0.6 \\
14\end{array}$ & $\begin{array}{l}0.12 \\
4\end{array}$ & $\begin{array}{l}-4.6 \\
17 \mathrm{E}- \\
02\end{array}$ & $\begin{array}{l}-0.3 \\
82\end{array}$ & $\begin{array}{l}-0.1 \\
22\end{array}$ & $\begin{array}{l}2.18 \\
1 \mathrm{E}-0 \\
2\end{array}$ & $\mid \begin{array}{l}-0.1 \\
91\end{array}$ & $\begin{array}{l}2.63 \\
7 \mathrm{E}-0 \\
2\end{array}$ & $\begin{array}{l}-2.7 \\
44 \mathrm{E}- \\
02\end{array}$ & $\begin{array}{l}0.34 \\
3\end{array}$ & $\begin{array}{l}-0.1 \\
20\end{array}$ & \begin{tabular}{|l}
-7.4 \\
$97 \mathrm{E}-$ \\
03
\end{tabular} & $\begin{array}{l}2.05 \\
2 \mathrm{E}-0 \\
2\end{array}$ & $\begin{array}{l}-0.1 \\
67\end{array}$ & $\begin{array}{l}4.29 \\
1 \mathrm{E}-0 \\
3\end{array}$ & $\begin{array}{l}-7.8 \\
13 \mathrm{E}- \\
02\end{array}$ & $\begin{array}{l}-1.3 \\
75 \mathrm{E}- \\
02\end{array}$ & $\begin{array}{l}-0.1 \\
77\end{array}$ & $\begin{array}{l}-0.2 \\
18\end{array}$ & \begin{tabular}{|l}
3.44 \\
$5 \mathrm{E}-0$ \\
2
\end{tabular} & $\begin{array}{l}-3.9 \\
38 \mathrm{E}- \\
02\end{array}$ & $\begin{array}{l}1.89 \\
8 \mathrm{E}-0 \\
2\end{array}$ \\
\hline & V5 & \begin{tabular}{|l}
-2.6 \\
$64 \mathrm{E}-$ \\
02
\end{tabular} & $\begin{array}{l}-4.6 \\
62 \mathrm{E}- \\
02\end{array}$ & $\begin{array}{l}-6.4 \\
21 \mathrm{E}- \\
02\end{array}$ & $\begin{array}{l}-0.6 \\
14\end{array}$ & $\begin{array}{l}0.81 \\
8\end{array}$ & $\begin{array}{l}1.41 \\
5 \mathrm{E}-0 \\
2\end{array}$ & $\begin{array}{l}-6.1 \\
41 \mathrm{E}- \\
03\end{array}$ & $\begin{array}{l}-7.2 \\
32 \mathrm{E}- \\
02\end{array}$ & $\begin{array}{l}-0.1 \\
09\end{array}$ & \begin{tabular}{|l}
-7.3 \\
$34 \mathrm{E}-$ \\
03
\end{tabular} & $\begin{array}{l}-0.1 \\
61\end{array}$ & $\begin{array}{l}1.50 \\
1 \mathrm{E}-0 \\
2\end{array}$ & $\begin{array}{l}0.32 \\
5\end{array}$ & $\begin{array}{l}-0.1 \\
84\end{array}$ & $\begin{array}{l}-0.1 \\
32\end{array}$ & $\begin{array}{l}-1.5 \\
41 \mathrm{E}- \\
02\end{array}$ & $\begin{array}{l}3.50 \\
0 \mathrm{E}-0 \\
2\end{array}$ & $\begin{array}{l}-0.3 \\
06\end{array}$ & $\begin{array}{l}-0.3 \\
26\end{array}$ & $\begin{array}{l}2.67 \\
2 \mathrm{E}-0 \\
2\end{array}$ & $\begin{array}{l}-4.5 \\
15 \mathrm{E}- \\
02\end{array}$ & $\begin{array}{l}2.56 \\
3 \mathrm{E}-0 \\
2\end{array}$ & $\begin{array}{l}0.14 \\
3\end{array}$ & $\begin{array}{l}-1.4 \\
90 \mathrm{E}- \\
02\end{array}$ & $\begin{array}{l}5.96 \\
6 \mathrm{E}-0 \\
2\end{array}$ & $\begin{array}{l}-0.1 \\
11\end{array}$ \\
\hline & V6 & $\begin{array}{l}0.16 \\
4\end{array}$ & $\begin{array}{l}-0.1 \\
40\end{array}$ & $\begin{array}{l}5.74 \\
7 \mathrm{E}-0 \\
2\end{array}$ & $\begin{array}{l}0.12 \\
4\end{array}$ & $\begin{array}{l}1.41 \\
5 \mathrm{E}-0 \\
2\end{array}$ & $\begin{array}{l}0.85 \\
4\end{array}$ & $\begin{array}{l}-0.4 \\
51\end{array}$ & $\begin{array}{l}-0.3 \\
83\end{array}$ & $\begin{array}{l}-0.1 \\
78\end{array}$ & $\begin{array}{l}9.76 \\
3 \mathrm{E}-0 \\
3\end{array}$ & $\begin{array}{l}0.12 \\
2\end{array}$ & $\begin{array}{l}6.11 \\
1 \mathrm{E}-0 \\
3\end{array}$ & $\begin{array}{l}-9.9 \\
43 \mathrm{E}- \\
03\end{array}$ & $\begin{array}{l}2.45 \\
8 \mathrm{E}-0 \\
2\end{array}$ & \begin{tabular}{|l}
-6.1 \\
$54 \mathrm{E}-$ \\
02
\end{tabular} & \begin{tabular}{|l}
-6.3 \\
$28 \mathrm{E}-$ \\
03
\end{tabular} & $\begin{array}{l}2.82 \\
3 \mathrm{E}-0 \\
2\end{array}$ & \begin{tabular}{|l}
-5.3 \\
$14 \mathrm{E}-$ \\
02
\end{tabular} & $\begin{array}{l}1.06 \\
4 \mathrm{E}-0 \\
2\end{array}$ & $\begin{array}{l}-0.1 \\
22\end{array}$ & \begin{tabular}{|l}
-8.6 \\
$94 \mathrm{E}-$ \\
02
\end{tabular} & $\begin{array}{l}-5.3 \\
54 \mathrm{E}- \\
02\end{array}$ & \begin{tabular}{|l|}
-5.8 \\
$77 \mathrm{E}-$ \\
02 \\
\end{tabular} & $\begin{array}{l}7.66 \\
3 \mathrm{E}-0 \\
2\end{array}$ & $\begin{array}{l}-2.9 \\
50 \mathrm{E}- \\
02\end{array}$ & $\begin{array}{l}5.86 \\
7 \mathrm{E}-0 \\
2\end{array}$ \\
\hline & V7 & \begin{tabular}{|l}
-2.9 \\
$39 \mathrm{E}-$ \\
02
\end{tabular} & $\begin{array}{l}3.35 \\
2 \mathrm{E}-0 \\
2\end{array}$ & \begin{tabular}{|l}
-8.2 \\
$81 \mathrm{E}-$ \\
02
\end{tabular} & \begin{tabular}{|l}
-4.6 \\
$17 \mathrm{E}-$ \\
02
\end{tabular} & $\begin{array}{l}-6.1 \\
41 \mathrm{E}- \\
03\end{array}$ & $\begin{array}{l}-0.4 \\
51\end{array}$ & $\begin{array}{l}0.81 \\
6\end{array}$ & $\begin{array}{l}0.19 \\
8\end{array}$ & $\begin{array}{l}5.03 \\
5 \mathrm{E}-0 \\
2\end{array}$ & $\begin{array}{l}-4.1 \\
54 \mathrm{E}- \\
02\end{array}$ & $\begin{array}{l}-0.7 \\
35\end{array}$ & $\begin{array}{l}-4.3 \\
73 \mathrm{E}- \\
02\end{array}$ & $\begin{array}{l}6.36 \\
6 \mathrm{E}-0 \\
2\end{array}$ & $\begin{array}{l}-2.5 \\
95 \mathrm{E}- \\
02\end{array}$ & $\begin{array}{l}0.10 \\
6\end{array}$ & $\begin{array}{l}1.22 \\
6 \mathrm{E}-0 \\
2\end{array}$ & \begin{tabular}{|l}
-2.1 \\
$76 \mathrm{E}-$ \\
02
\end{tabular} & \begin{tabular}{|l}
-9.7 \\
$29 \mathrm{E}-$ \\
03
\end{tabular} & $\begin{array}{l}-1.6 \\
99 \mathrm{E}- \\
04\end{array}$ & $\begin{array}{l}0.10 \\
1\end{array}$ & $\begin{array}{l}1.53 \\
2 \mathrm{E}-0 \\
2\end{array}$ & $\begin{array}{l}3.58 \\
6 \mathrm{E}-0 \\
2\end{array}$ & \begin{tabular}{|l}
5.47 \\
$4 \mathrm{E}-0$ \\
2
\end{tabular} & \begin{tabular}{|l|}
-4.1 \\
$13 \mathrm{E}-$ \\
02
\end{tabular} & $\begin{array}{l}4.16 \\
2 \mathrm{E}-0 \\
2\end{array}$ & \begin{tabular}{|l}
-3.9 \\
$05 \mathrm{E}-$ \\
02
\end{tabular} \\
\hline & V8 & $\begin{array}{l}-0.3 \\
40\end{array}$ & $\begin{array}{l}-0.1 \\
46\end{array}$ & \begin{tabular}{|l}
-5.5 \\
$03 \mathrm{E}-$ \\
03
\end{tabular} & $\begin{array}{l}-0.3 \\
82\end{array}$ & $\begin{array}{l}-7.2 \\
32 \mathrm{E}- \\
02\end{array}$ & $\begin{array}{l}-0.3 \\
83\end{array}$ & $\begin{array}{l}0.19 \\
8\end{array}$ & $\begin{array}{l}0.79 \\
0\end{array}$ & $\begin{array}{l}0.19 \\
8\end{array}$ & $\begin{array}{l}4.41 \\
4 \mathrm{E}-0 \\
3\end{array}$ & $\begin{array}{l}-4.6 \\
47 \mathrm{E}- \\
02\end{array}$ & $\begin{array}{l}-2.7 \\
13 \mathrm{E}- \\
02\end{array}$ & $\begin{array}{l}3.97 \\
2 \mathrm{E}-0 \\
2\end{array}$ & $\begin{array}{l}-9.6 \\
90 \mathrm{E}- \\
02\end{array}$ & $\begin{array}{l}9.49 \\
0 \mathrm{E}-0 \\
2\end{array}$ & \begin{tabular}{|l}
-1.0 \\
$20 \mathrm{E}-$ \\
02
\end{tabular} & $\begin{array}{l}-1.8 \\
82 \mathrm{E}- \\
02\end{array}$ & $\begin{array}{l}5.65 \\
1 \mathrm{E}-0 \\
2\end{array}$ & $\begin{array}{l}-2.4 \\
33 \mathrm{E}- \\
03\end{array}$ & $\begin{array}{l}-0.1 \\
30\end{array}$ & $\begin{array}{l}0.16 \\
9\end{array}$ & $\begin{array}{l}0.19 \\
9\end{array}$ & $\begin{array}{l}0.21 \\
8\end{array}$ & \begin{tabular}{|l|}
-4.7 \\
$66 \mathrm{E}-$ \\
02
\end{tabular} & $\begin{array}{l}-1.2 \\
21 \mathrm{E}- \\
02\end{array}$ & $\begin{array}{l}6.21 \\
1 \mathrm{E}-0 \\
3\end{array}$ \\
\hline & V9 & $\begin{array}{l}6.74 \\
3 \mathrm{E}-0 \\
2 \\
\end{array}$ & $\begin{array}{l}0.11 \\
9\end{array}$ & $\begin{array}{l}0.25 \\
7\end{array}$ & $\begin{array}{l}-0.1 \\
22\end{array}$ & $\begin{array}{l}-0.1 \\
09\end{array}$ & $\begin{array}{l}-0.1 \\
78\end{array}$ & $\begin{array}{l}5.03 \\
5 \mathrm{E}-0 \\
2 \\
\end{array}$ & $\begin{array}{l}0.19 \\
8\end{array}$ & $\begin{array}{l}0.85 \\
2\end{array}$ & $\begin{array}{l}6.36 \\
6 \mathrm{E}-0 \\
2 \\
\end{array}$ & \begin{tabular}{|l}
-3.0 \\
$87 \mathrm{E}-$ \\
02
\end{tabular} & \begin{tabular}{|l}
-7.9 \\
$08 \mathrm{E}-$ \\
02
\end{tabular} & $\begin{array}{l}3.85 \\
7 \mathrm{E}-0 \\
2\end{array}$ & \begin{tabular}{|l}
5.34 \\
$1 \mathrm{E}-0$ \\
2 \\
\end{tabular} & \begin{tabular}{|l}
-3.7 \\
$69 \mathrm{E}-$ \\
02 \\
\end{tabular} & \begin{tabular}{|l}
-2.2 \\
$98 \mathrm{E}-$ \\
02
\end{tabular} & $\begin{array}{l}-0.1 \\
32\end{array}$ & $\begin{array}{l}5.23 \\
3 \mathrm{E}-0 \\
2 \\
\end{array}$ & $\begin{array}{l}-0.2 \\
02\end{array}$ & $\begin{array}{l}-0.1 \\
91\end{array}$ & $\begin{array}{l}2.89 \\
8 \mathrm{E}-0 \\
3\end{array}$ & $\begin{array}{l}7.90 \\
6 \mathrm{E}-0 \\
2\end{array}$ & \begin{tabular}{|l|}
5.22 \\
$1 \mathrm{E}-0$ \\
2
\end{tabular} & $\begin{array}{l}-0.1 \\
63\end{array}$ & $\begin{array}{l}5.12 \\
5 \mathrm{E}-0 \\
2\end{array}$ & \begin{tabular}{|l}
-6.3 \\
$73 \mathrm{E}-$ \\
02
\end{tabular} \\
\hline & V10 & \begin{tabular}{|l}
-6.0 \\
$47 \mathrm{E}$ \\
03 \\
\end{tabular} & $\begin{array}{l}4.22 \\
2 \mathrm{E}-0 \\
2\end{array}$ & $\begin{array}{l}1.40 \\
0 \mathrm{E}-0 \\
2\end{array}$ & $\begin{array}{l}2.18 \\
1 \mathrm{E}-0 \\
2 \\
\end{array}$ & \begin{tabular}{|l}
-7.3 \\
$34 \mathrm{E}-$ \\
03
\end{tabular} & \begin{tabular}{|l}
9.76 \\
$3 \mathrm{E}-0$ \\
3 \\
\end{tabular} & \begin{tabular}{|l}
-4.1 \\
$54 \mathrm{E}-$ \\
02
\end{tabular} & $\begin{array}{l}4.41 \\
4 \mathrm{E}-0 \\
3\end{array}$ & $\begin{array}{l}6.36 \\
6 \mathrm{E}-0 \\
2\end{array}$ & $\begin{array}{l}0.88 \\
7\end{array}$ & $\begin{array}{l}-1.5 \\
24 \mathrm{E}- \\
02\end{array}$ & $-\begin{array}{l}-0.8 \\
12\end{array}$ & $\begin{array}{l}0.32 \\
4\end{array}$ & $\begin{array}{l}7.05 \\
7 \mathrm{E}-0 \\
2\end{array}$ & $\begin{array}{l}1.12 \\
9 \mathrm{E}-0 \\
2\end{array}$ & $\begin{array}{l}3.76 \\
4 \mathrm{E}-0 \\
4\end{array}$ & $\begin{array}{l}1.08 \\
9 \mathrm{E}-0 \\
2\end{array}$ & \begin{tabular}{|l}
-4.8 \\
$94 \mathrm{E}-$ \\
02
\end{tabular} & $\begin{array}{l}-3.6 \\
22 \mathrm{E}- \\
02\end{array}$ & $\begin{array}{l}-1.4 \\
52 \mathrm{E}- \\
02\end{array}$ & $\begin{array}{l}2.92 \\
6 \mathrm{E}-0 \\
2\end{array}$ & \begin{tabular}{|l}
-1.4 \\
$24 \mathrm{E}-$ \\
02
\end{tabular} & \begin{tabular}{|l}
-1.4 \\
$00 \mathrm{E}-$ \\
02 \\
\end{tabular} & \begin{tabular}{|l}
-2.7 \\
$97 \mathrm{E}-$ \\
02 \\
\end{tabular} & $\begin{array}{l}1.17 \\
9 \mathrm{E}-0 \\
2\end{array}$ & \begin{tabular}{|l}
-2.8 \\
$76 \mathrm{E}-$ \\
02 \\
\end{tabular} \\
\hline & V11 & \begin{tabular}{|l|}
-5.2 \\
$02 \mathrm{E}-$ \\
02 \\
\end{tabular} & \begin{tabular}{|l}
-7.2 \\
$63 \mathrm{E}-$ \\
02 \\
\end{tabular} & $\begin{array}{l}9.42 \\
0 \mathrm{E}-0 \\
2\end{array}$ & \begin{tabular}{|l}
-0.1 \\
91
\end{tabular} & $\begin{array}{l}-0.1 \\
61\end{array}$ & $\begin{array}{l}0.12 \\
2\end{array}$ & $\begin{array}{l}-0.7 \\
35\end{array}$ & \begin{tabular}{|l}
-4.6 \\
$47 \mathrm{E}-$ \\
02
\end{tabular} & \begin{tabular}{|l}
-3.0 \\
$87 \mathrm{E}-$ \\
02
\end{tabular} & $\begin{array}{l}-1.5 \\
24 \mathrm{E}- \\
02\end{array}$ & $\left\{\begin{array}{l}0.79 \\
7\end{array}\right.$ & $\begin{array}{l}4.47 \\
9 \mathrm{E}-0 \\
2\end{array}$ & $\begin{array}{l}-0.3 \\
25\end{array}$ & \begin{tabular}{|l}
-4.3 \\
$79 \mathrm{E}-$ \\
02
\end{tabular} & \begin{tabular}{|l}
-7.5 \\
$87 \mathrm{E}-$ \\
02 \\
\end{tabular} & \begin{tabular}{|l}
-2.4 \\
$14 \mathrm{E}-$ \\
03
\end{tabular} & $\begin{array}{l}-1.3 \\
90 \mathrm{E}- \\
02\end{array}$ & $\begin{array}{l}0.28 \\
5\end{array}$ & $\begin{array}{l}0.35 \\
3\end{array}$ & $\begin{array}{l}3.52 \\
6 \mathrm{E}-0 \\
2\end{array}$ & $\begin{array}{l}1.54 \\
9 \mathrm{E}-0 \\
2\end{array}$ & $\begin{array}{l}7.95 \\
6 \mathrm{E}-0 \\
2\end{array}$ & \begin{tabular}{|l|}
-5.3 \\
$45 \mathrm{E}-$ \\
02 \\
\end{tabular} & $\begin{array}{l}3.14 \\
8 \mathrm{E}-0 \\
2\end{array}$ & \begin{tabular}{|l}
-2.5 \\
$14 \mathrm{E}-$ \\
02 \\
\end{tabular} & $\begin{array}{l}6.36 \\
8 \mathrm{E}-0 \\
2 \\
\end{array}$ \\
\hline & V12 & $\begin{array}{l}4.12 \\
9 \mathrm{E}-0 \\
3 \\
\end{array}$ & $\begin{array}{l}-2.0 \\
77 \mathrm{E}- \\
02\end{array}$ & \begin{tabular}{|l|}
-3.1 \\
$56 \mathrm{E}-$ \\
02
\end{tabular} & $\begin{array}{l}2.63 \\
7 \mathrm{E}-0 \\
2\end{array}$ & $\begin{array}{l}1.50 \\
1 \mathrm{E}-0 \\
2\end{array}$ & $\begin{array}{l}6.11 \\
1 \mathrm{E}-0 \\
3\end{array}$ & $\begin{array}{l}-4.3 \\
73 \mathrm{E}- \\
02\end{array}$ & $\begin{array}{l}-2.7 \\
13 \mathrm{E}- \\
02\end{array}$ & \begin{tabular}{|l}
-7.9 \\
$08 \mathrm{E}-$ \\
02
\end{tabular} & $\begin{array}{l}-0.8 \\
12\end{array}$ & \begin{tabular}{|l}
4.47 \\
$9 \mathrm{E}-0$ \\
2
\end{tabular} & $\begin{array}{l}0.87 \\
6\end{array}$ & $\begin{array}{l}-0.5 \\
33\end{array}$ & $\begin{array}{l}-9.7 \\
51 \mathrm{E}- \\
04\end{array}$ & $\begin{array}{l}-4.1 \\
44 \mathrm{E}- \\
02\end{array}$ & $\begin{array}{l}1.07 \\
3 \mathrm{E}-0 \\
2\end{array}$ & $\begin{array}{l}-1.8 \\
27 \mathrm{E}- \\
02\end{array}$ & \begin{tabular}{|l}
-4.7 \\
$80 \mathrm{E}-$ \\
02
\end{tabular} & $\begin{array}{l}5.20 \\
1 \mathrm{E}-0 \\
2\end{array}$ & $\begin{array}{l}4.84 \\
3 \mathrm{E}-0 \\
3\end{array}$ & $\begin{array}{l}-4.6 \\
73 \mathrm{E}- \\
03\end{array}$ & $\begin{array}{l}7.25 \\
0 \mathrm{E}-0 \\
3\end{array}$ & \begin{tabular}{|l|}
-6.0 \\
$36 \mathrm{E}-$ \\
03 \\
\end{tabular} & $\begin{array}{l}1.51 \\
8 \mathrm{E}-0 \\
2\end{array}$ & $\begin{array}{l}1.64 \\
8 \mathrm{E}-0 \\
3\end{array}$ & \begin{tabular}{|l}
-5.0 \\
$17 \mathrm{E}-$ \\
03 \\
\end{tabular} \\
\hline & V13 & \begin{tabular}{|l|}
-0.1 \\
00
\end{tabular} & $\begin{array}{l}0.12 \\
6\end{array}$ & \begin{tabular}{|l}
-9.2 \\
$10 \mathrm{E}-$ \\
02
\end{tabular} & \begin{tabular}{|l}
-2.7 \\
$44 \mathrm{E}-$ \\
02
\end{tabular} & 0.32 & \begin{tabular}{|l}
-9.9 \\
$43 \mathrm{E}-$ \\
03
\end{tabular} & $\begin{array}{l}6.36 \\
6 \mathrm{E}-0 \\
2\end{array}$ & $\begin{array}{l}3.97 \\
2 \mathrm{E}-0 \\
2\end{array}$ & $\begin{array}{l}3.85 \\
7 \mathrm{E}-0 \\
2\end{array}$ & $\begin{array}{l}0.32 \\
4\end{array}$ & $\begin{array}{l}-0.3 \\
25\end{array}$ & $\begin{array}{l}-0.5 \\
33\end{array}$ & $\begin{array}{l}0.82 \\
1\end{array}$ & $\begin{array}{l}-0.3 \\
39\end{array}$ & $\begin{array}{l}3.12 \\
9 \mathrm{E}-0 \\
2\end{array}$ & \begin{tabular}{|l}
5.00 \\
$5 \mathrm{E}-0$ \\
4
\end{tabular} & \begin{tabular}{|l}
1.76 \\
$8 \mathrm{E}-0$ \\
3
\end{tabular} & $\begin{array}{l}-0.6 \\
47\end{array}$ & $\begin{array}{l}-0.4 \\
56\end{array}$ & \begin{tabular}{|l|}
-0.1 \\
77
\end{tabular} & $\begin{array}{l}-0.1 \\
27\end{array}$ & $\begin{array}{l}6.35 \\
1 \mathrm{E}-0 \\
2\end{array}$ & $\mid \begin{array}{l}0.20 \\
9\end{array}$ & \begin{tabular}{|l|}
-2.9 \\
$29 \mathrm{E}-$ \\
02 \\
\end{tabular} & $\begin{array}{l}6.41 \\
3 \mathrm{E}-0 \\
2\end{array}$ & $\begin{array}{l}-0.1 \\
27\end{array}$ \\
\hline
\end{tabular}


(Table 2 continued)

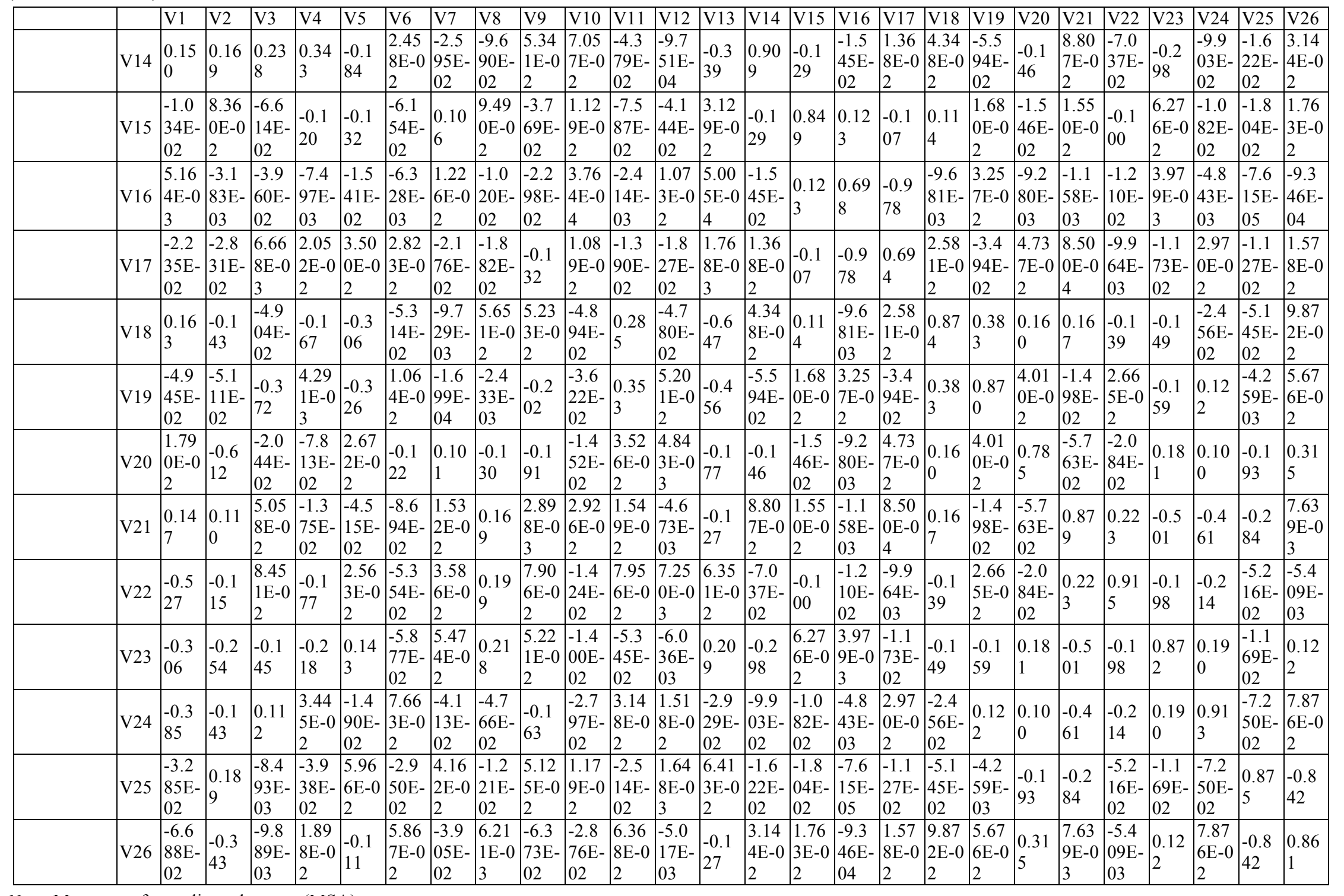

Note. Measures of sampling adequacy (MSA). 
Table 3

Correlation Matrix

\begin{tabular}{|c|c|c|c|c|c|c|c|c|c|c|c|c|c|c|c|c|c|c|c|c|c|c|c|c|c|c|c|}
\hline & & V1 & $\mathrm{V} 2$ & V3 & $\mathrm{V} 4$ & V5 & V6 & V7 & V8 & 9 & 10 & 11 & $V 12$ & V13 & V14 & V15 & V16 & V17 & V18 & V19 & $\mathrm{V} 20$ & V21 & V22 & V23 & V24 & $\mathrm{V} 25$ & $\mathrm{~V} 26$ \\
\hline \multirow[t]{17}{*}{ Correlation } & V1 & $\begin{array}{l}1.00 \\
0\end{array}$ & $\begin{array}{l}0.73 \\
2\end{array}$ & $\begin{array}{l}0.40 \\
7\end{array}$ & $\begin{array}{l}0.04 \\
1\end{array}$ & $\begin{array}{l}0.05 \\
9\end{array}$ & \begin{tabular}{|l|}
-0.1 \\
99
\end{tabular} & \begin{tabular}{|l|}
-0.1 \\
72
\end{tabular} & $\begin{array}{l}0.16 \\
6\end{array}$ & $\begin{array}{l}-0.0 \\
32\end{array}$ & \begin{tabular}{l|}
0.29 \\
7
\end{tabular} & \begin{tabular}{|l|}
-0.1 \\
21
\end{tabular} & $\begin{array}{l}0.30 \\
9\end{array}$ & $\begin{array}{l}0.31 \\
2\end{array}$ & \begin{tabular}{|l|}
0.31 \\
6
\end{tabular} & \begin{tabular}{|l|}
0.02 \\
0
\end{tabular} & $\begin{array}{l}0.29 \\
1\end{array}$ & $\begin{array}{l}0.29 \\
0\end{array}$ & $\begin{array}{l}0.27 \\
4\end{array}$ & $\begin{array}{l}0.46 \\
2\end{array}$ & $\begin{array}{l}0.16 \\
8\end{array}$ & $\begin{array}{l}0.75 \\
4\end{array}$ & $\begin{array}{l}0.93 \\
8\end{array}$ & $\begin{array}{l}0.86 \\
2\end{array}$ & $\begin{array}{l}0.90 \\
2\end{array}$ & $\begin{array}{l}0.83 \\
6\end{array}$ & $\begin{array}{l}0.82 \\
3\end{array}$ \\
\hline & V2 & $\begin{array}{l}0.73 \\
2\end{array}$ & $\begin{array}{l}1.00 \\
0\end{array}$ & \begin{tabular}{|l}
0.36 \\
0
\end{tabular} & \begin{tabular}{|l}
0.06 \\
1
\end{tabular} & $\begin{array}{l}0.09 \\
2\end{array}$ & $\begin{array}{l}0.06 \\
7\end{array}$ & $\begin{array}{l}-0.0 \\
97\end{array}$ & $\begin{array}{l}0.31 \\
4\end{array}$ & $\begin{array}{l}0.00 \\
2\end{array}$ & $\begin{array}{l}0.28 \\
6\end{array}$ & \begin{tabular}{|l|}
-0.0 \\
64
\end{tabular} & 0.30 & $\begin{array}{l}0.30 \\
7\end{array}$ & $\begin{array}{l}0.28 \\
7\end{array}$ & \begin{tabular}{|l|}
-0.0 \\
59
\end{tabular} & $\begin{array}{l}0.24 \\
4\end{array}$ & \begin{tabular}{|l}
0.24 \\
0
\end{tabular} & $\begin{array}{l}0.29 \\
3\end{array}$ & 0.40 & $\begin{array}{l}0.51 \\
8\end{array}$ & $\begin{array}{l}0.54 \\
8\end{array}$ & $\begin{array}{l}0.73 \\
2\end{array}$ & $\begin{array}{l}0.66 \\
2\end{array}$ & $\begin{array}{l}0.68 \\
5\end{array}$ & $\begin{array}{l}0.65 \\
8\end{array}$ & $\begin{array}{l}0.67 \\
6\end{array}$ \\
\hline & V3 & $\begin{array}{l}0.40 \\
7\end{array}$ & \begin{tabular}{|l}
0.36 \\
0
\end{tabular} & $\begin{array}{l}1.00 \\
0\end{array}$ & $\begin{array}{l}0.30 \\
3\end{array}$ & $\begin{array}{l}0.29 \\
9\end{array}$ & $\begin{array}{l}0.12 \\
5\end{array}$ & $\begin{array}{l}0.16 \\
6\end{array}$ & $\begin{array}{l}0.20 \\
9\end{array}$ & \begin{tabular}{|l|}
-0.1 \\
60
\end{tabular} & $\begin{array}{l}0.70 \\
0\end{array}$ & $\begin{array}{l}0.15 \\
1\end{array}$ & \begin{tabular}{|l|}
0.74 \\
1
\end{tabular} & $\begin{array}{l}0.75 \\
5\end{array}$ & $\begin{array}{l}0.61 \\
7\end{array}$ & $\begin{array}{l}0.04 \\
5\end{array}$ & \begin{tabular}{|l|}
-0.0 \\
49
\end{tabular} & \begin{tabular}{|l|}
-0.0 \\
54
\end{tabular} & $\begin{array}{l}0.74 \\
1\end{array}$ & 0.81 & $\begin{array}{l}0.38 \\
3\end{array}$ & $\begin{array}{l}0.36 \\
4\end{array}$ & $\begin{array}{l}0.42 \\
5\end{array}$ & $\begin{array}{l}0.48 \\
1\end{array}$ & $\begin{array}{l}0.34 \\
1\end{array}$ & \begin{tabular}{|l|}
0.43 \\
4
\end{tabular} & $\begin{array}{l}0.46 \\
5\end{array}$ \\
\hline & V4 & \begin{tabular}{|l|}
0.04 \\
1
\end{tabular} & $\begin{array}{l}0.06 \\
1\end{array}$ & $\begin{array}{l}0.30 \\
3\end{array}$ & $\begin{array}{l}1.00 \\
0\end{array}$ & $\begin{array}{l}0.93 \\
2\end{array}$ & $\begin{array}{l}0.54 \\
3\end{array}$ & $\begin{array}{l}0.68 \\
5\end{array}$ & $\begin{array}{l}0.68 \\
6\end{array}$ & \begin{tabular}{|l|}
0.38 \\
7
\end{tabular} & $\begin{array}{l}0.35 \\
0\end{array}$ & $\begin{array}{l}0.74 \\
5\end{array}$ & $\begin{array}{l}0.35 \\
9\end{array}$ & $\begin{array}{l}0.35 \\
2\end{array}$ & $\begin{array}{l}0.17 \\
9\end{array}$ & $\begin{array}{l}0.45 \\
9\end{array}$ & $\begin{array}{l}0.16 \\
8\end{array}$ & $\begin{array}{l}0.16 \\
7\end{array}$ & $\begin{array}{l}0.44 \\
3\end{array}$ & $\begin{array}{l}0.25 \\
9\end{array}$ & $\begin{array}{l}0.23 \\
9\end{array}$ & $\begin{array}{l}0.01 \\
2\end{array}$ & $\begin{array}{l}0.11 \\
0\end{array}$ & $\begin{array}{l}0.06 \\
3\end{array}$ & \begin{tabular}{|l|}
0.04 \\
7
\end{tabular} & $\begin{array}{l}0.10 \\
7\end{array}$ & $\begin{array}{l}0.13 \\
4\end{array}$ \\
\hline & V5 & $\begin{array}{l}0.05 \\
9\end{array}$ & $\begin{array}{l}0.09 \\
2\end{array}$ & $\begin{array}{l}0.29 \\
9\end{array}$ & $\begin{array}{l}0.93 \\
2\end{array}$ & $\begin{array}{l}1.00 \\
0\end{array}$ & $\begin{array}{l}0.51 \\
3\end{array}$ & $\begin{array}{l}0.63 \\
5\end{array}$ & $\begin{array}{l}0.66 \\
7\end{array}$ & $\begin{array}{l}0.40 \\
0\end{array}$ & $\begin{array}{l}0.31 \\
3\end{array}$ & $\begin{array}{l}0.69 \\
3\end{array}$ & \begin{tabular}{|l|}
0.32 \\
0
\end{tabular} & $\begin{array}{l}0.31 \\
3\end{array}$ & $\begin{array}{l}0.17 \\
6\end{array}$ & $\begin{array}{l}0.47 \\
5\end{array}$ & $\begin{array}{l}0.18 \\
1\end{array}$ & \begin{tabular}{|l}
0.17 \\
9
\end{tabular} & $\begin{array}{l}0.40 \\
7\end{array}$ & $\begin{array}{l}0.28 \\
5\end{array}$ & $\begin{array}{l}0.22 \\
7\end{array}$ & $\begin{array}{l}0.02 \\
1\end{array}$ & $\begin{array}{l}0.11 \\
8\end{array}$ & $\begin{array}{l}0.06 \\
6\end{array}$ & $\begin{array}{l}0.06 \\
1\end{array}$ & $\begin{array}{l}0.12 \\
0\end{array}$ & $\begin{array}{l}0.15 \\
3\end{array}$ \\
\hline & V6 & \begin{tabular}{|l|}
-0.1 \\
99 \\
\end{tabular} & $\begin{array}{l}0.06 \\
7 \\
\end{array}$ & $\begin{array}{l}0.12 \\
5\end{array}$ & $\begin{array}{l}0.54 \\
3\end{array}$ & $\begin{array}{l}0.51 \\
3\end{array}$ & $\begin{array}{l}1.00 \\
0\end{array}$ & $\begin{array}{l}0.74 \\
3\end{array}$ & $\begin{array}{l}0.55 \\
1\end{array}$ & $\begin{array}{l}0.30 \\
7\end{array}$ & $\begin{array}{l}0.33 \\
8\end{array}$ & $\begin{array}{l}0.68 \\
9\end{array}$ & $\begin{array}{l}0.35 \\
5\end{array}$ & $\begin{array}{l}0.34 \\
8\end{array}$ & $\begin{array}{l}0.26 \\
0\end{array}$ & $\begin{array}{l}0.19 \\
5\end{array}$ & $\begin{array}{l}0.05 \\
8\end{array}$ & $\begin{array}{l}0.05 \\
6\end{array}$ & $\begin{array}{l}0.37 \\
2\end{array}$ & \begin{tabular}{|l|}
0.11 \\
1
\end{tabular} & $\begin{array}{l}0.43 \\
5\end{array}$ & \begin{tabular}{|l|}
-0.1 \\
53
\end{tabular} & \begin{tabular}{|l|}
-0.1 \\
54
\end{tabular} & \begin{tabular}{|l|}
-0.1 \\
75
\end{tabular} & \begin{tabular}{|l|}
-0.1 \\
46 \\
\end{tabular} & \begin{tabular}{|l|}
-0.1 \\
20
\end{tabular} & \begin{tabular}{|l|}
-0.0 \\
94
\end{tabular} \\
\hline & V7 & \begin{tabular}{|l|}
-0.1 \\
72 \\
\end{tabular} & \begin{tabular}{|l|}
-0.0 \\
97 \\
\end{tabular} & $\begin{array}{l}0.16 \\
6 \\
\end{array}$ & $\begin{array}{l}0.68 \\
5\end{array}$ & $\begin{array}{l}0.63 \\
5\end{array}$ & \begin{tabular}{|l}
0.74 \\
3 \\
\end{tabular} & $\begin{array}{l}1.00 \\
0\end{array}$ & $\begin{array}{l}0.43 \\
0\end{array}$ & $\begin{array}{l}0.32 \\
2\end{array}$ & $\begin{array}{l}0.42 \\
6 \\
\end{array}$ & $\begin{array}{l}0.94 \\
2\end{array}$ & \begin{tabular}{|l}
0.43 \\
3 \\
\end{tabular} & $\begin{array}{l}0.41 \\
8 \\
\end{array}$ & $\begin{array}{l}0.30 \\
7\end{array}$ & $\begin{array}{l}0.26 \\
8\end{array}$ & $\begin{array}{l}0.09 \\
6\end{array}$ & $\begin{array}{l}0.09 \\
6\end{array}$ & $\begin{array}{l}0.43 \\
6\end{array}$ & $\begin{array}{l}0.09 \\
5\end{array}$ & $\begin{array}{l}0.21 \\
1\end{array}$ & \begin{tabular}{|l|}
0.1 \\
02
\end{tabular} & \begin{tabular}{|l}
0.1 \\
37 \\
\end{tabular} & \begin{tabular}{|l|}
0.1 \\
45 \\
\end{tabular} & \begin{tabular}{|l|}
-0.1 \\
01 \\
\end{tabular} & $\begin{array}{l}-0.0 \\
76\end{array}$ & \begin{tabular}{|l|}
-0.0 \\
39 \\
\end{tabular} \\
\hline & V8 & $\begin{array}{l}0.16 \\
6 \\
\end{array}$ & \begin{tabular}{|l|}
0.31 \\
4 \\
\end{tabular} & $\begin{array}{l}0.20 \\
9 \\
\end{array}$ & $\begin{array}{l}0.68 \\
6 \\
\end{array}$ & $\begin{array}{l}0.66 \\
7 \\
\end{array}$ & \begin{tabular}{|l}
0.55 \\
1 \\
\end{tabular} & $\begin{array}{l}0.43 \\
0 \\
\end{array}$ & $\begin{array}{l}1.00 \\
0\end{array}$ & $\begin{array}{l}0.25 \\
5 \\
\end{array}$ & $\begin{array}{l}0.22 \\
3 \\
\end{array}$ & $\begin{array}{l}0.49 \\
2 \\
\end{array}$ & \begin{tabular}{|l|}
0.22 \\
9 \\
\end{tabular} & \begin{tabular}{|l}
0.22 \\
1 \\
\end{tabular} & $\begin{array}{l}0.10 \\
9 \\
\end{array}$ & $\begin{array}{l}0.26 \\
6 \\
\end{array}$ & $\begin{array}{l}0.19 \\
0\end{array}$ & $\begin{array}{l}0.18 \\
6 \\
\end{array}$ & $\begin{array}{l}0.27 \\
9 \\
\end{array}$ & $\begin{array}{l}0.18 \\
2 \\
\end{array}$ & $\begin{array}{l}0.42 \\
5 \\
\end{array}$ & \begin{tabular}{|l|}
-0.0 \\
67 \\
\end{tabular} & $\begin{array}{l}0.15 \\
7 \\
\end{array}$ & $\begin{array}{l}0.02 \\
0\end{array}$ & \begin{tabular}{|l}
0.10 \\
1 \\
\end{tabular} & $\begin{array}{l}0.10 \\
6 \\
\end{array}$ & \begin{tabular}{|l}
0.13 \\
4 \\
\end{tabular} \\
\hline & V9 & \begin{tabular}{|l|}
-0.0 \\
32 \\
\end{tabular} & $\begin{array}{l}0.00 \\
2\end{array}$ & \begin{tabular}{|l|}
-0.1 \\
60 \\
\end{tabular} & $\begin{array}{l}0.38 \\
7\end{array}$ & $\begin{array}{l}0.40 \\
0\end{array}$ & $\begin{array}{l}0.30 \\
7\end{array}$ & $\begin{array}{l}0.32 \\
2\end{array}$ & $\begin{array}{l}0.25 \\
5\end{array}$ & $\begin{array}{l}1.00 \\
0\end{array}$ & \begin{tabular}{|l|}
-0.1 \\
33 \\
\end{tabular} & $\begin{array}{l}0.36 \\
3\end{array}$ & \begin{tabular}{|l|}
-0.1 \\
10 \\
\end{tabular} & \begin{tabular}{|l|}
-0.1 \\
18
\end{tabular} & \begin{tabular}{|l|}
-0.1 \\
48
\end{tabular} & $\begin{array}{l}0.25 \\
9\end{array}$ & $\begin{array}{l}0.73 \\
3\end{array}$ & \begin{tabular}{|l}
0.73 \\
8
\end{tabular} & $\begin{array}{l}-0.1 \\
41\end{array}$ & \begin{tabular}{l|}
0.00 \\
9
\end{tabular} & $\begin{array}{l}0.05 \\
7\end{array}$ & $\begin{array}{l}0.01 \\
0\end{array}$ & \begin{tabular}{|l|}
-0.0 \\
40
\end{tabular} & \begin{tabular}{|l|}
-0.0 \\
53
\end{tabular} & $\begin{array}{l}0.00 \\
5\end{array}$ & \begin{tabular}{|l|}
-0.0 \\
15 \\
\end{tabular} & \begin{tabular}{|l|}
-0.0 \\
20
\end{tabular} \\
\hline & V10 & \begin{tabular}{|l|}
0.29 \\
7 \\
\end{tabular} & $\begin{array}{l}0.28 \\
6 \\
\end{array}$ & \begin{tabular}{|l|}
0.70 \\
0
\end{tabular} & $\begin{array}{l}0.35 \\
0\end{array}$ & $\begin{array}{l}0.31 \\
3\end{array}$ & $\begin{array}{l}0.33 \\
8\end{array}$ & $\begin{array}{l}0.42 \\
6\end{array}$ & & \begin{tabular}{|l|}
-0.1 \\
33 \\
\end{tabular} & $\begin{array}{l}1.00 \\
0\end{array}$ & $\begin{array}{l}0.40 \\
5\end{array}$ & $\begin{array}{l}0.96 \\
6 \\
\end{array}$ & $\begin{array}{l}0.91 \\
8\end{array}$ & $\begin{array}{l}0.79 \\
7\end{array}$ & $\begin{array}{l}0.03 \\
5\end{array}$ & \begin{tabular}{|l|}
-0.1 \\
66
\end{tabular} & \begin{tabular}{|l|}
-0.1 \\
70
\end{tabular} & $\begin{array}{l}0.90 \\
8\end{array}$ & $\begin{array}{l}0.68 \\
5\end{array}$ & $\begin{array}{l}0.46 \\
9\end{array}$ & $\begin{array}{l}0.31 \\
8\end{array}$ & $\begin{array}{l}0.34 \\
9 \\
\end{array}$ & $\begin{array}{l}0.34 \\
4\end{array}$ & \begin{tabular}{|l|}
0.33 \\
9 \\
\end{tabular} & $\begin{array}{l}0.38 \\
8\end{array}$ & \begin{tabular}{|l|}
0.43 \\
0
\end{tabular} \\
\hline & V11 & \begin{tabular}{|l|}
-0.1 \\
21 \\
\end{tabular} & \begin{tabular}{|l|}
-0.0 \\
64 \\
\end{tabular} & \begin{tabular}{|l}
0.15 \\
1 \\
\end{tabular} & $\begin{array}{l}0.74 \\
5\end{array}$ & $\begin{array}{l}0.69 \\
3\end{array}$ & $\begin{array}{l}0.68 \\
9\end{array}$ & & & \begin{tabular}{|l}
0.36 \\
3 \\
\end{tabular} & $\begin{array}{l}0.40 \\
5\end{array}$ & $\begin{array}{l}1.00 \\
0\end{array}$ & \begin{tabular}{|l|}
0.41 \\
9 \\
\end{tabular} & $\begin{array}{l}0.41 \\
2\end{array}$ & $\begin{array}{l}0.30 \\
2\end{array}$ & $\begin{array}{l}0.33 \\
0\end{array}$ & \begin{tabular}{|l}
0.14 \\
1
\end{tabular} & \begin{tabular}{|l}
0.14 \\
1
\end{tabular} & $\begin{array}{l}0.42 \\
7\end{array}$ & & $\begin{array}{l}0.22 \\
8\end{array}$ & \begin{tabular}{|l|}
-0.0 \\
73 \\
\end{tabular} & \begin{tabular}{|l}
-0.0 \\
91
\end{tabular} & \begin{tabular}{|l|}
-0.1 \\
11 \\
\end{tabular} & \begin{tabular}{|l}
-0.0 \\
58 \\
\end{tabular} & $\begin{array}{l}-0.0 \\
36\end{array}$ & \begin{tabular}{|l|}
-0.0 \\
03
\end{tabular} \\
\hline & V12 & \begin{tabular}{|l}
0.30 \\
9 \\
\end{tabular} & \begin{tabular}{|l}
0.30 \\
5 \\
\end{tabular} & \begin{tabular}{|l}
0.74 \\
1 \\
\end{tabular} & $\begin{array}{l}0.35 \\
9\end{array}$ & \begin{tabular}{|l}
0.32 \\
0
\end{tabular} & $\begin{array}{l}0.35 \\
5 \\
\end{array}$ & \begin{tabular}{|l}
0.43 \\
3 \\
\end{tabular} & & \begin{tabular}{|l|}
-0.1 \\
10 \\
\end{tabular} & $\begin{array}{l}0.96 \\
6 \\
\end{array}$ & \begin{tabular}{|l}
0.41 \\
9 \\
\end{tabular} & \begin{tabular}{|l|}
1.00 \\
0 \\
\end{tabular} & $\begin{array}{l}0.97 \\
8 \\
\end{array}$ & \begin{tabular}{|l}
0.86 \\
4 \\
\end{tabular} & $\begin{array}{l}0.03 \\
4 \\
\end{array}$ & \begin{tabular}{|l}
-0.1 \\
46 \\
\end{tabular} & $\begin{array}{l}-0.1 \\
50 \\
\end{array}$ & $\begin{array}{l}0.95 \\
5 \\
\end{array}$ & & & $\begin{array}{l}0.34 \\
5 \\
\end{array}$ & $\begin{array}{l}0.36 \\
2 \\
\end{array}$ & $\begin{array}{l}0.36 \\
5 \\
\end{array}$ & & $\begin{array}{l}0.40 \\
7 \\
\end{array}$ & \begin{tabular}{|l}
0.44 \\
8 \\
\end{tabular} \\
\hline & V13 & \begin{tabular}{|l|}
0.31 \\
2 \\
\end{tabular} & $\begin{array}{l}0.30 \\
7 \\
\end{array}$ & $\begin{array}{l}0.75 \\
5 \\
\end{array}$ & $\begin{array}{l}0.35 \\
2\end{array}$ & $\begin{array}{l}0.31 \\
3\end{array}$ & $\begin{array}{l}0.34 \\
8\end{array}$ & $\begin{array}{l}0.41 \\
8\end{array}$ & $\begin{array}{l}0.22 \\
1\end{array}$ & \begin{tabular}{|l|}
-0.1 \\
18 \\
\end{tabular} & $\begin{array}{l}0.91 \\
8\end{array}$ & $\begin{array}{l}0.41 \\
2\end{array}$ & $\begin{array}{l}0.97 \\
8 \\
\end{array}$ & $\begin{array}{l}1.00 \\
0\end{array}$ & $\begin{array}{l}0.89 \\
6\end{array}$ & $\begin{array}{l}0.02 \\
5\end{array}$ & \begin{tabular}{|l|}
-0.1 \\
52
\end{tabular} & \begin{tabular}{|l|}
-0.1 \\
56
\end{tabular} & $\begin{array}{l}0.96 \\
6\end{array}$ & \begin{tabular}{l|}
0.77 \\
6 \\
\end{tabular} & $\begin{array}{l}0.53 \\
3 \\
\end{array}$ & $\begin{array}{l}0.35 \\
8\end{array}$ & $\begin{array}{l}0.36 \\
3 \\
\end{array}$ & $\begin{array}{l}0.37 \\
3 \\
\end{array}$ & \begin{tabular}{|l}
0.36 \\
0 \\
\end{tabular} & $\begin{array}{l}0.41 \\
2 \\
\end{array}$ & \begin{tabular}{|l}
0.45 \\
0 \\
\end{tabular} \\
\hline & V14 & $\begin{array}{l}0.31 \\
6 \\
\end{array}$ & \begin{tabular}{|l}
0.28 \\
7 \\
\end{tabular} & \begin{tabular}{|l|}
0.61 \\
7 \\
\end{tabular} & $\begin{array}{l}0.17 \\
9\end{array}$ & $\begin{array}{l}0.17 \\
6\end{array}$ & $\begin{array}{l}0.26 \\
0\end{array}$ & $\begin{array}{l}0.30 \\
7\end{array}$ & $\begin{array}{l}0.10 \\
9\end{array}$ & \begin{tabular}{|l|}
-0.1 \\
48 \\
\end{tabular} & $\begin{array}{l}0.79 \\
7\end{array}$ & $\begin{array}{l}0.30 \\
2\end{array}$ & \begin{tabular}{|l}
0.86 \\
4 \\
\end{tabular} & $\begin{array}{l}0.89 \\
6\end{array}$ & $\begin{array}{l}1.00 \\
0\end{array}$ & $\begin{array}{l}0.01 \\
4\end{array}$ & \begin{tabular}{|l|}
-0.1 \\
54
\end{tabular} & \begin{tabular}{|l|}
-0.1 \\
57
\end{tabular} & $\begin{array}{l}0.84 \\
1\end{array}$ & $\begin{array}{l}0.70 \\
3 \\
\end{array}$ & $\begin{array}{l}0.49 \\
6\end{array}$ & $\begin{array}{l}0.37 \\
9\end{array}$ & $\begin{array}{l}0.36 \\
7\end{array}$ & $\begin{array}{l}0.40 \\
7\end{array}$ & $\begin{array}{l}0.38 \\
1 \\
\end{array}$ & $\begin{array}{l}0.39 \\
0\end{array}$ & $\begin{array}{l}0.40 \\
8 \\
\end{array}$ \\
\hline & V15 & \begin{tabular}{|l|}
0.02 \\
0
\end{tabular} & \begin{tabular}{|l}
-0.0 \\
59
\end{tabular} & $\begin{array}{l}0.04 \\
5\end{array}$ & \begin{tabular}{|l|}
0.45 \\
9
\end{tabular} & $\begin{array}{l}0.47 \\
5\end{array}$ & $\begin{array}{l}0.19 \\
5\end{array}$ & $\begin{array}{l}0.26 \\
8\end{array}$ & $\begin{array}{l}0.26 \\
6\end{array}$ & $\begin{array}{l}0.25 \\
9\end{array}$ & $\begin{array}{l}0.03 \\
5\end{array}$ & $\begin{array}{l}0.33 \\
0\end{array}$ & $\begin{array}{l}0.03 \\
4\end{array}$ & $\begin{array}{l}0.02 \\
5\end{array}$ & $\begin{array}{l}0.01 \\
4\end{array}$ & $\begin{array}{l}1.00 \\
0\end{array}$ & $\begin{array}{l}0.10 \\
6\end{array}$ & $\begin{array}{l}0.11 \\
9\end{array}$ & $\begin{array}{l}0.05 \\
4\end{array}$ & $\begin{array}{l}0.04 \\
5\end{array}$ & $\begin{array}{l}0.01 \\
8\end{array}$ & \begin{tabular}{|l|}
-0.0 \\
06
\end{tabular} & $\begin{array}{l}0.06 \\
2\end{array}$ & $\begin{array}{l}0.00 \\
3\end{array}$ & $\begin{array}{l}0.02 \\
2\end{array}$ & $\begin{array}{l}0.03 \\
1\end{array}$ & $\begin{array}{l}0.02 \\
9\end{array}$ \\
\hline & V16 & \begin{tabular}{|l|}
0.29 \\
1 \\
\end{tabular} & $\begin{array}{l}0.24 \\
4 \\
\end{array}$ & \begin{tabular}{|l|}
-0.0 \\
49
\end{tabular} & $\begin{array}{l}0.16 \\
8\end{array}$ & $\begin{array}{l}0.18 \\
1\end{array}$ & $\begin{array}{l}0.05 \\
8\end{array}$ & $\begin{array}{l}0.09 \\
6\end{array}$ & $\begin{array}{l}0.19 \\
0\end{array}$ & \begin{tabular}{|l|}
0.73 \\
3 \\
\end{tabular} & \begin{tabular}{|l|}
-0.1 \\
66
\end{tabular} & $\begin{array}{l}0.14 \\
1\end{array}$ & \begin{tabular}{|l|}
-0.1 \\
46 \\
\end{tabular} & \begin{tabular}{|l|}
-0.1 \\
52
\end{tabular} & \begin{tabular}{|l|}
-0.1 \\
54
\end{tabular} & $\begin{array}{l}0.10 \\
6\end{array}$ & $\begin{array}{l}1.00 \\
0\end{array}$ & $\begin{array}{l}0.99 \\
4\end{array}$ & \begin{tabular}{|l|}
-0.1 \\
98
\end{tabular} & \begin{tabular}{l|}
0.05 \\
6
\end{tabular} & \begin{tabular}{|l|}
-0.0 \\
34
\end{tabular} & $\begin{array}{l}0.20 \\
7\end{array}$ & $\begin{array}{l}0.25 \\
2\end{array}$ & $\begin{array}{l}0.23 \\
2\end{array}$ & \begin{tabular}{|l|}
0.24 \\
0
\end{tabular} & $\begin{array}{l}0.21 \\
1\end{array}$ & $\begin{array}{l}0.19 \\
5\end{array}$ \\
\hline & V17 & \begin{tabular}{|l|}
0.29 \\
0
\end{tabular} & \begin{tabular}{|l}
0.24 \\
0
\end{tabular} & \begin{tabular}{|l|}
-0.0 \\
54
\end{tabular} & $\begin{array}{l}0.16 \\
7\end{array}$ & $\begin{array}{l}0.17 \\
9\end{array}$ & $\begin{array}{l}0.05 \\
6\end{array}$ & $\begin{array}{l}0.09 \\
6\end{array}$ & & $\begin{array}{l}0.73 \\
8\end{array}$ & \begin{tabular}{|l}
0.1 \\
70
\end{tabular} & $\begin{array}{l}0.14 \\
1\end{array}$ & \begin{tabular}{|l|}
-0.1 \\
50 \\
\end{tabular} & \begin{tabular}{|l|}
-0.1 \\
56 \\
\end{tabular} & $\begin{array}{l}-0.1 \\
57\end{array}$ & $\begin{array}{l}0.11 \\
9\end{array}$ & $\begin{array}{l}0.99 \\
4\end{array}$ & $\begin{array}{l}1.00 \\
0\end{array}$ & \begin{tabular}{|l|}
-0.2 \\
04
\end{tabular} & $\begin{array}{l}0.05 \\
5 \\
\end{array}$ & \begin{tabular}{|l|}
-0.0 \\
40
\end{tabular} & & $\begin{array}{l}0.25 \\
0\end{array}$ & $\begin{array}{l}0.23 \\
1\end{array}$ & & & $\begin{array}{l}0.19 \\
3\end{array}$ \\
\hline
\end{tabular}


(Table 3 continued)

\begin{tabular}{|c|c|c|c|c|c|c|c|c|c|c|c|c|c|c|c|c|c|c|c|c|c|c|c|c|c|c|c|}
\hline & & V1 & V2 & V3 & V4 & V5 & V6 & V7 & V8 & V9 & V10 & V11 & V12 & V13 & V14 & V15 & V16 & V17 & V18 & V19 & V20 & V21 & V22 & V23 & V24 & V25 & V26 \\
\hline & V18 & $\begin{array}{l}0.27 \\
4\end{array}$ & $\begin{array}{l}0.29 \\
3\end{array}$ & $\begin{array}{l}0.74 \\
1\end{array}$ & $\begin{array}{l}0.44 \\
3\end{array}$ & $\begin{array}{l}0.40 \\
7\end{array}$ & $\begin{array}{l}0.37 \\
2\end{array}$ & 6 & $\begin{array}{l}0.27 \\
9\end{array}$ & \begin{tabular}{|l|}
-0.1 \\
41 \\
\end{tabular} & $\begin{array}{l}0.90 \\
8\end{array}$ & $\begin{array}{l}0.42 \\
7\end{array}$ & $\begin{array}{l}0.95 \\
5\end{array}$ & $\begin{array}{l}0.96 \\
6\end{array}$ & $\begin{array}{l}0.84 \\
1\end{array}$ & $\begin{array}{l}0.05 \\
4\end{array}$ & \begin{tabular}{|l}
0.1 \\
98
\end{tabular} & \begin{tabular}{|l}
-0.2 \\
04
\end{tabular} & $\begin{array}{l}1.00 \\
0\end{array}$ & $\begin{array}{l}0.71 \\
8\end{array}$ & $\begin{array}{l}0.50 \\
6\end{array}$ & $\begin{array}{l}0.29 \\
3\end{array}$ & $\begin{array}{l}0.35 \\
3\end{array}$ & $\begin{array}{l}0.34 \\
7\end{array}$ & $\begin{array}{l}0.31 \\
9\end{array}$ & $\begin{array}{l}0.37 \\
1\end{array}$ & $\begin{array}{l}0.41 \\
2 \\
\end{array}$ \\
\hline & V19 & $\begin{array}{l}0.46 \\
2\end{array}$ & $\begin{array}{l}0.40 \\
6\end{array}$ & $\begin{array}{l}0.81 \\
7\end{array}$ & $\begin{array}{l}0.25 \\
9\end{array}$ & $\begin{array}{l}0.28 \\
5\end{array}$ & $\begin{array}{l}0.11 \\
1\end{array}$ & $\begin{array}{l}0.09 \\
5\end{array}$ & $\begin{array}{l}0.18 \\
2\end{array}$ & $\begin{array}{l}0.00 \\
9\end{array}$ & $\begin{array}{l}0.68 \\
5\end{array}$ & $\begin{array}{l}0.08 \\
9\end{array}$ & $\begin{array}{l}0.74 \\
6\end{array}$ & $\begin{array}{l}0.77 \\
6\end{array}$ & $\begin{array}{l}0.70 \\
3\end{array}$ & $\begin{array}{l}0.04 \\
5\end{array}$ & $\begin{array}{l}0.05 \\
6\end{array}$ & $\begin{array}{l}0.05 \\
5\end{array}$ & $\begin{array}{l}0.71 \\
8\end{array}$ & $\begin{array}{l}1.00 \\
0\end{array}$ & $\begin{array}{l}0.44 \\
3\end{array}$ & $\begin{array}{l}0.46 \\
2\end{array}$ & $\begin{array}{l}0.47 \\
9\end{array}$ & $\begin{array}{l}0.54 \\
2\end{array}$ & $\begin{array}{l}0.42 \\
4\end{array}$ & $\begin{array}{l}0.49 \\
2\end{array}$ & $\begin{array}{l}0.51 \\
2\end{array}$ \\
\hline & V20 & $\begin{array}{l}0.16 \\
8\end{array}$ & $\begin{array}{l}0.51 \\
8\end{array}$ & $\begin{array}{l}0.38 \\
3\end{array}$ & $\begin{array}{l}0.23 \\
9\end{array}$ & $\begin{array}{l}0.22 \\
7\end{array}$ & $\begin{array}{l}0.43 \\
5\end{array}$ & $\begin{array}{l}0.21 \\
1 \\
\end{array}$ & $\begin{array}{l}0.42 \\
5\end{array}$ & $\begin{array}{l}0.05 \\
7\end{array}$ & $\begin{array}{l}0.46 \\
9\end{array}$ & $\begin{array}{l}0.22 \\
8\end{array}$ & $\begin{array}{l}0.51 \\
5\end{array}$ & $\begin{array}{l}0.53 \\
3\end{array}$ & $\begin{array}{l}0.49 \\
6\end{array}$ & $\begin{array}{l}0.01 \\
8\end{array}$ & \begin{tabular}{|l|}
-0.0 \\
34 \\
\end{tabular} & $\begin{array}{l}-0.0 \\
40\end{array}$ & $\begin{array}{l}0.50 \\
6\end{array}$ & $\begin{array}{l}0.44 \\
3\end{array}$ & $\begin{array}{l}1.00 \\
0\end{array}$ & $\begin{array}{l}0.10 \\
8\end{array}$ & $\begin{array}{l}0.20 \\
4\end{array}$ & $\begin{array}{l}0.14 \\
0\end{array}$ & $\begin{array}{l}0.17 \\
4\end{array}$ & $\begin{array}{l}0.17 \\
8\end{array}$ & \begin{tabular}{|l}
0.17 \\
7
\end{tabular} \\
\hline & V21 & $\begin{array}{l}0.75 \\
4\end{array}$ & $\begin{array}{l}0.54 \\
8\end{array}$ & $\begin{array}{l}0.36 \\
4\end{array}$ & $\begin{array}{l}0.01 \\
2\end{array}$ & \begin{tabular}{|l}
0.02 \\
1
\end{tabular} & $\begin{array}{l}-0.1 \\
53\end{array}$ & \begin{tabular}{|l|}
-0.1 \\
02
\end{tabular} & \begin{tabular}{|l|}
-0.0 \\
67 \\
\end{tabular} & \begin{tabular}{|l|}
0.01 \\
0
\end{tabular} & $\begin{array}{l}0.31 \\
8\end{array}$ & $\begin{array}{l}-0.0 \\
73\end{array}$ & $\begin{array}{l}0.34 \\
5\end{array}$ & $\begin{array}{l}0.35 \\
8\end{array}$ & $\begin{array}{l}0.37 \\
9\end{array}$ & \begin{tabular}{|l|}
-0.0 \\
06
\end{tabular} & $\begin{array}{l}0.20 \\
7\end{array}$ & $\begin{array}{l}0.20 \\
7\end{array}$ & $\begin{array}{l}0.29 \\
3\end{array}$ & $\begin{array}{l}0.46 \\
2\end{array}$ & $\begin{array}{l}0.10 \\
8\end{array}$ & $\begin{array}{l}1.00 \\
0\end{array}$ & $\begin{array}{l}0.72 \\
3\end{array}$ & $\begin{array}{l}0.82 \\
9\end{array}$ & $\begin{array}{l}0.83 \\
9\end{array}$ & $\begin{array}{l}0.85 \\
7\end{array}$ & $\begin{array}{l}0.81 \\
4\end{array}$ \\
\hline & V22 & $\begin{array}{l}0.93 \\
8\end{array}$ & $\begin{array}{l}0.73 \\
2\end{array}$ & $\begin{array}{l}0.42 \\
5\end{array}$ & $\begin{array}{l}0.11 \\
0\end{array}$ & $\begin{array}{l}0.11 \\
8\end{array}$ & \begin{tabular}{|l}
-0.1 \\
54
\end{tabular} & \begin{tabular}{|l|}
-0.1 \\
37
\end{tabular} & $\begin{array}{l}0.15 \\
7\end{array}$ & \begin{tabular}{|l|}
-0.0 \\
40
\end{tabular} & $\begin{array}{l}0.34 \\
9\end{array}$ & \begin{tabular}{|l}
-0.0 \\
91
\end{tabular} & $\begin{array}{l}0.36 \\
2\end{array}$ & $\begin{array}{l}0.36 \\
3\end{array}$ & $\begin{array}{l}0.36 \\
7\end{array}$ & $\begin{array}{l}0.06 \\
2\end{array}$ & $\begin{array}{l}0.25 \\
2\end{array}$ & $\begin{array}{l}0.25 \\
0\end{array}$ & $\begin{array}{l}0.35 \\
3\end{array}$ & $\begin{array}{l}0.47 \\
9\end{array}$ & $\begin{array}{l}0.20 \\
4\end{array}$ & \begin{tabular}{|l}
0.72 \\
3
\end{tabular} & $\begin{array}{l}1.00 \\
0\end{array}$ & $\begin{array}{l}0.85 \\
9\end{array}$ & $\begin{array}{l}0.87 \\
9\end{array}$ & $\begin{array}{l}0.81 \\
8\end{array}$ & $\begin{array}{l}0.80 \\
6\end{array}$ \\
\hline & V23 & $\begin{array}{l}0.86 \\
2\end{array}$ & $\begin{array}{l}0.66 \\
2\end{array}$ & $\begin{array}{l}0.48 \\
1\end{array}$ & $\begin{array}{l}0.06 \\
3\end{array}$ & $\begin{array}{l}0.06 \\
6\end{array}$ & \begin{tabular}{|l|}
-0.1 \\
75
\end{tabular} & \begin{tabular}{|l|}
-0.1 \\
45
\end{tabular} & $\begin{array}{l}0.02 \\
0\end{array}$ & \begin{tabular}{|l|}
-0.0 \\
53
\end{tabular} & $\begin{array}{l}0.34 \\
4\end{array}$ & \begin{tabular}{|l|}
-0.1 \\
11
\end{tabular} & $\begin{array}{l}0.36 \\
5\end{array}$ & $\begin{array}{l}0.37 \\
3\end{array}$ & $\begin{array}{l}0.40 \\
7\end{array}$ & $\begin{array}{l}0.00 \\
3\end{array}$ & $\begin{array}{l}0.23 \\
2\end{array}$ & $\begin{array}{l}0.23 \\
1\end{array}$ & $\begin{array}{l}0.34 \\
7\end{array}$ & $\begin{array}{l}0.54 \\
2\end{array}$ & $\begin{array}{l}0.14 \\
0\end{array}$ & $\begin{array}{l}0.82 \\
9\end{array}$ & $\begin{array}{l}0.85 \\
9\end{array}$ & $\begin{array}{l}1.00 \\
0\end{array}$ & $\begin{array}{l}0.81 \\
6\end{array}$ & $\begin{array}{l}0.79 \\
5\end{array}$ & $\begin{array}{l}0.76 \\
8\end{array}$ \\
\hline & V24 & $\begin{array}{l}0.90 \\
2\end{array}$ & $\begin{array}{l}0.68 \\
5\end{array}$ & $\begin{array}{l}0.34 \\
1 \\
\end{array}$ & $\begin{array}{l}0.04 \\
7\end{array}$ & $\begin{array}{l}0.06 \\
1 \\
\end{array}$ & $\begin{array}{l}-0.1 \\
46 \\
\end{array}$ & \begin{tabular}{|l|}
-0.1 \\
01 \\
\end{tabular} & $\begin{array}{l}0.10 \\
1\end{array}$ & $\begin{array}{l}0.00 \\
5\end{array}$ & $\begin{array}{l}0.33 \\
9 \\
\end{array}$ & $\begin{array}{l}-0.0 \\
58 \\
\end{array}$ & $\begin{array}{l}0.35 \\
6\end{array}$ & $\begin{array}{l}0.36 \\
0\end{array}$ & $\begin{array}{l}0.38 \\
1\end{array}$ & $\begin{array}{l}0.02 \\
2 \\
\end{array}$ & $\begin{array}{l}0.24 \\
0\end{array}$ & $\begin{array}{l}0.23 \\
9 \\
\end{array}$ & $\begin{array}{l}0.31 \\
9 \\
\end{array}$ & $\begin{array}{l}0.42 \\
4\end{array}$ & $\begin{array}{l}0.17 \\
4\end{array}$ & $\begin{array}{l}0.83 \\
9 \\
\end{array}$ & $\begin{array}{l}0.87 \\
9\end{array}$ & $\begin{array}{l}0.81 \\
6\end{array}$ & $\begin{array}{l}1.00 \\
0\end{array}$ & $\begin{array}{l}0.85 \\
5 \\
\end{array}$ & \begin{tabular}{|l}
0.83 \\
0
\end{tabular} \\
\hline & V25 & $\begin{array}{l}0.83 \\
6 \\
\end{array}$ & $\begin{array}{l}0.65 \\
8 \\
\end{array}$ & $\begin{array}{l}0.43 \\
4\end{array}$ & $\begin{array}{l}0.10 \\
7\end{array}$ & \begin{tabular}{|l}
0.12 \\
0
\end{tabular} & \begin{tabular}{|l}
-0.1 \\
20 \\
\end{tabular} & \begin{tabular}{|l|}
-0.0 \\
76 \\
\end{tabular} & $\begin{array}{l}0.10 \\
6 \\
\end{array}$ & \begin{tabular}{|l}
-0.0 \\
15 \\
\end{tabular} & $\begin{array}{l}0.38 \\
8 \\
\end{array}$ & \begin{tabular}{|l}
-0.0 \\
36 \\
\end{tabular} & $\begin{array}{l}0.40 \\
7\end{array}$ & $\begin{array}{l}0.41 \\
2 \\
\end{array}$ & \begin{tabular}{|l}
0.39 \\
0 \\
\end{tabular} & \begin{tabular}{|l}
0.03 \\
1 \\
\end{tabular} & \begin{tabular}{|l}
0.21 \\
1 \\
\end{tabular} & $\begin{array}{l}0.20 \\
9 \\
\end{array}$ & $\begin{array}{l}0.37 \\
1 \\
\end{array}$ & $\begin{array}{l}0.49 \\
2 \\
\end{array}$ & $\begin{array}{l}0.17 \\
8 \\
\end{array}$ & $\begin{array}{l}0.85 \\
7\end{array}$ & $\begin{array}{l}0.81 \\
8 \\
\end{array}$ & $\begin{array}{l}0.79 \\
5\end{array}$ & $\begin{array}{l}0.85 \\
5 \\
\end{array}$ & $\begin{array}{l}1.00 \\
0\end{array}$ & $\begin{array}{l}0.96 \\
8 \\
\end{array}$ \\
\hline & V26 & $\begin{array}{l}0.82 \\
3\end{array}$ & $\begin{array}{l}0.67 \\
6\end{array}$ & $\begin{array}{l}0.46 \\
5\end{array}$ & $\begin{array}{l}0.13 \\
4\end{array}$ & $\begin{array}{l}0.15 \\
3\end{array}$ & \begin{tabular}{|l}
-0.0 \\
94
\end{tabular} & \begin{tabular}{|l}
-0.0 \\
39
\end{tabular} & $\begin{array}{l}0.13 \\
4\end{array}$ & \begin{tabular}{|l|}
-0.0 \\
20 \\
\end{tabular} & $\begin{array}{l}0.43 \\
0\end{array}$ & \begin{tabular}{|l|}
-0.0 \\
03 \\
\end{tabular} & $\begin{array}{l}0.44 \\
8 \\
\end{array}$ & $\begin{array}{l}0.45 \\
0\end{array}$ & $\begin{array}{l}0.40 \\
8 \\
\end{array}$ & $\begin{array}{l}0.02 \\
9 \\
\end{array}$ & $\begin{array}{l}0.19 \\
5\end{array}$ & \begin{tabular}{|l|}
0.19 \\
3 \\
\end{tabular} & $\begin{array}{l}0.41 \\
2\end{array}$ & $\begin{array}{l}0.51 \\
2\end{array}$ & $\begin{array}{l}0.17 \\
7 \\
\end{array}$ & $\begin{array}{l}0.81 \\
4\end{array}$ & $\begin{array}{l}0.80 \\
6 \\
\end{array}$ & $\begin{array}{l}0.76 \\
8 \\
\end{array}$ & $\begin{array}{l}0.83 \\
0\end{array}$ & $\begin{array}{l}0.96 \\
8 \\
\end{array}$ & $\begin{array}{l}1.00 \\
0\end{array}$ \\
\hline $\begin{array}{l}\text { Sig. } \\
\text { (1-tailed) }\end{array}$ & V1 & & $\begin{array}{l}0.00 \\
0\end{array}$ & $\begin{array}{l}0.00 \\
0 \\
\end{array}$ & $\begin{array}{l}0.21 \\
3 \\
\end{array}$ & $\begin{array}{l}0.12 \\
4 \\
\end{array}$ & $\begin{array}{l}0.00 \\
0\end{array}$ & $\begin{array}{l}0.00 \\
0 \\
\end{array}$ & $\begin{array}{l}0.00 \\
1 \\
\end{array}$ & $\begin{array}{l}0.26 \\
7 \\
\end{array}$ & $\begin{array}{l}0.00 \\
0 \\
\end{array}$ & $\begin{array}{l}0.00 \\
9 \\
\end{array}$ & $\begin{array}{l}0.00 \\
0\end{array}$ & $\begin{array}{l}0.00 \\
0 \\
\end{array}$ & $\begin{array}{l}0.00 \\
0 \\
\end{array}$ & $\begin{array}{l}0.35 \\
0 \\
\end{array}$ & $\begin{array}{l}0.00 \\
0 \\
\end{array}$ & $\begin{array}{l}0.00 \\
0 \\
\end{array}$ & $\begin{array}{l}0.00 \\
0 \\
\end{array}$ & $\begin{array}{l}0.00 \\
0 \\
\end{array}$ & $\begin{array}{l}0.00 \\
0 \\
\end{array}$ & $\begin{array}{l}0.00 \\
0\end{array}$ & $\begin{array}{l}0.00 \\
0\end{array}$ & $\begin{array}{l}0.00 \\
0 \\
\end{array}$ & $\begin{array}{l}0.00 \\
0 \\
\end{array}$ & $\begin{array}{l}0.00 \\
0 \\
\end{array}$ & $\begin{array}{l}0.00 \\
0\end{array}$ \\
\hline & V2 & $\begin{array}{l}0.00 \\
0\end{array}$ & & $\begin{array}{l}0.00 \\
0\end{array}$ & $\begin{array}{l}0.11 \\
5\end{array}$ & $\begin{array}{l}0.03 \\
5 \\
\end{array}$ & $\begin{array}{l}0.09 \\
3\end{array}$ & $\begin{array}{l}0.02 \\
8\end{array}$ & $\begin{array}{l}0.00 \\
0\end{array}$ & \begin{tabular}{|l}
0.48 \\
3 \\
\end{tabular} & $\begin{array}{l}0.00 \\
0\end{array}$ & $\begin{array}{l}0.10 \\
7\end{array}$ & $\begin{array}{l}0.00 \\
0\end{array}$ & $\begin{array}{l}0.00 \\
0\end{array}$ & $\begin{array}{l}0.00 \\
0\end{array}$ & $\begin{array}{l}0.12 \\
3 \\
\end{array}$ & $\begin{array}{l}0.00 \\
0\end{array}$ & $\begin{array}{l}0.00 \\
0\end{array}$ & $\begin{array}{l}0.00 \\
0\end{array}$ & $\begin{array}{l}0.00 \\
0\end{array}$ & $\begin{array}{l}0.00 \\
0\end{array}$ & $\begin{array}{l}0.00 \\
0\end{array}$ & $\begin{array}{l}0.00 \\
0\end{array}$ & $\begin{array}{l}0.00 \\
0\end{array}$ & $\begin{array}{l}0.00 \\
0\end{array}$ & $\begin{array}{l}0.00 \\
0\end{array}$ & $\begin{array}{l}0.00 \\
0\end{array}$ \\
\hline & V3 & $\begin{array}{l}0.00 \\
0\end{array}$ & $\begin{array}{l}0.00 \\
0\end{array}$ & & $\begin{array}{l}0.00 \\
0\end{array}$ & $\begin{array}{l}0.00 \\
0\end{array}$ & $\begin{array}{l}0.00 \\
7\end{array}$ & $\begin{array}{l}0.00 \\
1\end{array}$ & $\begin{array}{l}0.00 \\
0\end{array}$ & $\begin{array}{l}0.00 \\
1\end{array}$ & $\begin{array}{l}0.00 \\
0\end{array}$ & $\begin{array}{l}0.00 \\
1\end{array}$ & $\begin{array}{l}0.00 \\
0\end{array}$ & $\begin{array}{l}0.00 \\
0\end{array}$ & $\begin{array}{l}0.00 \\
0\end{array}$ & $\begin{array}{l}0.19 \\
1\end{array}$ & $\begin{array}{l}0.16 \\
8\end{array}$ & $\begin{array}{l}0.14 \\
7\end{array}$ & $\begin{array}{l}0.00 \\
0\end{array}$ & $\begin{array}{l}0.00 \\
0\end{array}$ & $\begin{array}{l}0.00 \\
0\end{array}$ & $\begin{array}{l}0.00 \\
0\end{array}$ & $\begin{array}{l}0.00 \\
0\end{array}$ & $\begin{array}{l}0.00 \\
0\end{array}$ & $\begin{array}{l}0.00 \\
0\end{array}$ & $\begin{array}{l}0.00 \\
0\end{array}$ & \begin{tabular}{|l|}
0.00 \\
0
\end{tabular} \\
\hline & V4 & $\begin{array}{l}0.21 \\
3 \\
\end{array}$ & $\begin{array}{l}0.11 \\
5\end{array}$ & $\begin{array}{l}0.00 \\
0\end{array}$ & & $\begin{array}{l}0.00 \\
0\end{array}$ & $\begin{array}{l}0.00 \\
0\end{array}$ & $\begin{array}{l}0.00 \\
0 \\
\end{array}$ & $\begin{array}{l}0.00 \\
0\end{array}$ & $\begin{array}{l}0.00 \\
0\end{array}$ & $\begin{array}{l}0.00 \\
0\end{array}$ & $\begin{array}{l}0.00 \\
0\end{array}$ & $\begin{array}{l}0.00 \\
0\end{array}$ & $\begin{array}{l}0.00 \\
0\end{array}$ & $\begin{array}{l}0.00 \\
0\end{array}$ & $\begin{array}{l}0.00 \\
0\end{array}$ & $\begin{array}{l}0.00 \\
0\end{array}$ & \begin{tabular}{|l|}
0.00 \\
1 \\
\end{tabular} & $\begin{array}{l}0.00 \\
0\end{array}$ & $\begin{array}{l}0.00 \\
0\end{array}$ & $\begin{array}{l}0.00 \\
0\end{array}$ & $\begin{array}{l}0.40 \\
7\end{array}$ & $\begin{array}{l}0.01 \\
6\end{array}$ & $\begin{array}{l}0.11 \\
0\end{array}$ & $\begin{array}{l}0.18 \\
0\end{array}$ & $\begin{array}{l}0.01 \\
8\end{array}$ & $\begin{array}{l}0.00 \\
4\end{array}$ \\
\hline & V5 & $\begin{array}{l}0.12 \\
4\end{array}$ & $\begin{array}{l}0.03 \\
5\end{array}$ & $\begin{array}{l}0.00 \\
0\end{array}$ & $\begin{array}{l}0.00 \\
0\end{array}$ & & $\begin{array}{l}0.00 \\
0\end{array}$ & $\begin{array}{l}0.00 \\
0 \\
\end{array}$ & $\begin{array}{l}0.00 \\
0\end{array}$ & $\begin{array}{l}0.00 \\
0\end{array}$ & \begin{tabular}{|l}
0.00 \\
0
\end{tabular} & $\begin{array}{l}0.00 \\
0\end{array}$ & $\begin{array}{l}0.00 \\
0\end{array}$ & $\begin{array}{l}0.00 \\
0\end{array}$ & $\begin{array}{l}0.00 \\
0\end{array}$ & $\begin{array}{l}0.00 \\
0\end{array}$ & & $\begin{array}{l}0.00 \\
0\end{array}$ & $\begin{array}{l}0.00 \\
0\end{array}$ & $\begin{array}{l}0.00 \\
0\end{array}$ & $\begin{array}{l}0.00 \\
0\end{array}$ & \begin{tabular}{|l|}
0.34 \\
1
\end{tabular} & $\begin{array}{l}0.01 \\
0\end{array}$ & $\begin{array}{l}0.09 \\
7\end{array}$ & $\begin{array}{l}0.11 \\
6\end{array}$ & $\begin{array}{l}0.00 \\
9\end{array}$ & \begin{tabular}{|l}
0.00 \\
1 \\
\end{tabular} \\
\hline & V6 & $\begin{array}{l}0.00 \\
0\end{array}$ & $\begin{array}{l}0.09 \\
3\end{array}$ & $\begin{array}{l}0.00 \\
7\end{array}$ & $\begin{array}{l}0.00 \\
0\end{array}$ & $\begin{array}{l}0.00 \\
0\end{array}$ & & $\begin{array}{l}0.00 \\
0\end{array}$ & $\begin{array}{l}0.00 \\
0\end{array}$ & $\begin{array}{l}0.00 \\
0\end{array}$ & $\begin{array}{l}0.00 \\
0\end{array}$ & $\begin{array}{l}0.00 \\
0\end{array}$ & $\begin{array}{l}0.00 \\
0\end{array}$ & $\begin{array}{l}0.00 \\
0\end{array}$ & $\begin{array}{l}0.00 \\
0\end{array}$ & $\begin{array}{l}0.00 \\
0\end{array}$ & $\begin{array}{l}0.12 \\
8\end{array}$ & $\begin{array}{l}0.13 \\
7\end{array}$ & $\begin{array}{l}0.00 \\
0\end{array}$ & $\begin{array}{l}0.01 \\
5\end{array}$ & $\begin{array}{l}0.00 \\
0\end{array}$ & $\begin{array}{l}0.00 \\
1\end{array}$ & $\begin{array}{l}0.00 \\
1\end{array}$ & $\begin{array}{l}0.00 \\
0\end{array}$ & $\begin{array}{l}0.00 \\
2\end{array}$ & $\begin{array}{l}0.00 \\
9\end{array}$ & $\begin{array}{l}0.03 \\
3\end{array}$ \\
\hline & V7 & $\begin{array}{l}0.00 \\
0\end{array}$ & $\begin{array}{l}0.02 \\
8\end{array}$ & $\begin{array}{l}0.00 \\
1\end{array}$ & $\begin{array}{l}0.00 \\
0\end{array}$ & $\begin{array}{l}0.00 \\
0\end{array}$ & $\begin{array}{l}0.00 \\
0\end{array}$ & & $\begin{array}{l}0.00 \\
0\end{array}$ & $\begin{array}{l}0.00 \\
0\end{array}$ & $\begin{array}{l}0.00 \\
0\end{array}$ & $\begin{array}{l}0.00 \\
0\end{array}$ & $\begin{array}{l}0.00 \\
0\end{array}$ & $\begin{array}{l}0.00 \\
0\end{array}$ & $\begin{array}{l}0.00 \\
0\end{array}$ & $\begin{array}{l}0.00 \\
0\end{array}$ & & & $\begin{array}{l}0.00 \\
0\end{array}$ & $\begin{array}{l}0.03 \\
2\end{array}$ & $\begin{array}{l}0.00 \\
0\end{array}$ & $\begin{array}{l}0.02 \\
3\end{array}$ & $\begin{array}{l}0.00 \\
4\end{array}$ & $\begin{array}{l}0.00 \\
2\end{array}$ & $\begin{array}{l}0.02 \\
3 \\
\end{array}$ & $\begin{array}{l}0.06 \\
9\end{array}$ & $\begin{array}{l}0.22 \\
5 \\
\end{array}$ \\
\hline & V8 & $\begin{array}{l}0.00 \\
1\end{array}$ & $\begin{array}{l}0.00 \\
0\end{array}$ & $\begin{array}{l}0.00 \\
0\end{array}$ & $\begin{array}{l}0.00 \\
0\end{array}$ & \begin{tabular}{|l}
0.00 \\
0
\end{tabular} & $\begin{array}{l}0.00 \\
0\end{array}$ & $\begin{array}{l}0.00 \\
0\end{array}$ & & $\begin{array}{l}0.00 \\
0\end{array}$ & \begin{tabular}{|l}
0.00 \\
0
\end{tabular} & $\begin{array}{l}0.00 \\
0\end{array}$ & $\begin{array}{l}0.00 \\
0\end{array}$ & $\begin{array}{l}0.00 \\
0\end{array}$ & $\begin{array}{l}0.01 \\
6\end{array}$ & $\begin{array}{l}0.00 \\
0\end{array}$ & & & $\begin{array}{l}0.00 \\
0\end{array}$ & $\begin{array}{l}0.00 \\
0\end{array}$ & $\begin{array}{l}0.00 \\
0\end{array}$ & $\begin{array}{l}0.09 \\
5\end{array}$ & $\begin{array}{l}0.00 \\
1\end{array}$ & $\begin{array}{l}0.34 \\
7\end{array}$ & $\begin{array}{l}0.02 \\
4\end{array}$ & $\begin{array}{l}0.01 \\
9\end{array}$ & $\begin{array}{l}0.00 \\
4\end{array}$ \\
\hline
\end{tabular}


(Table 3 continued)

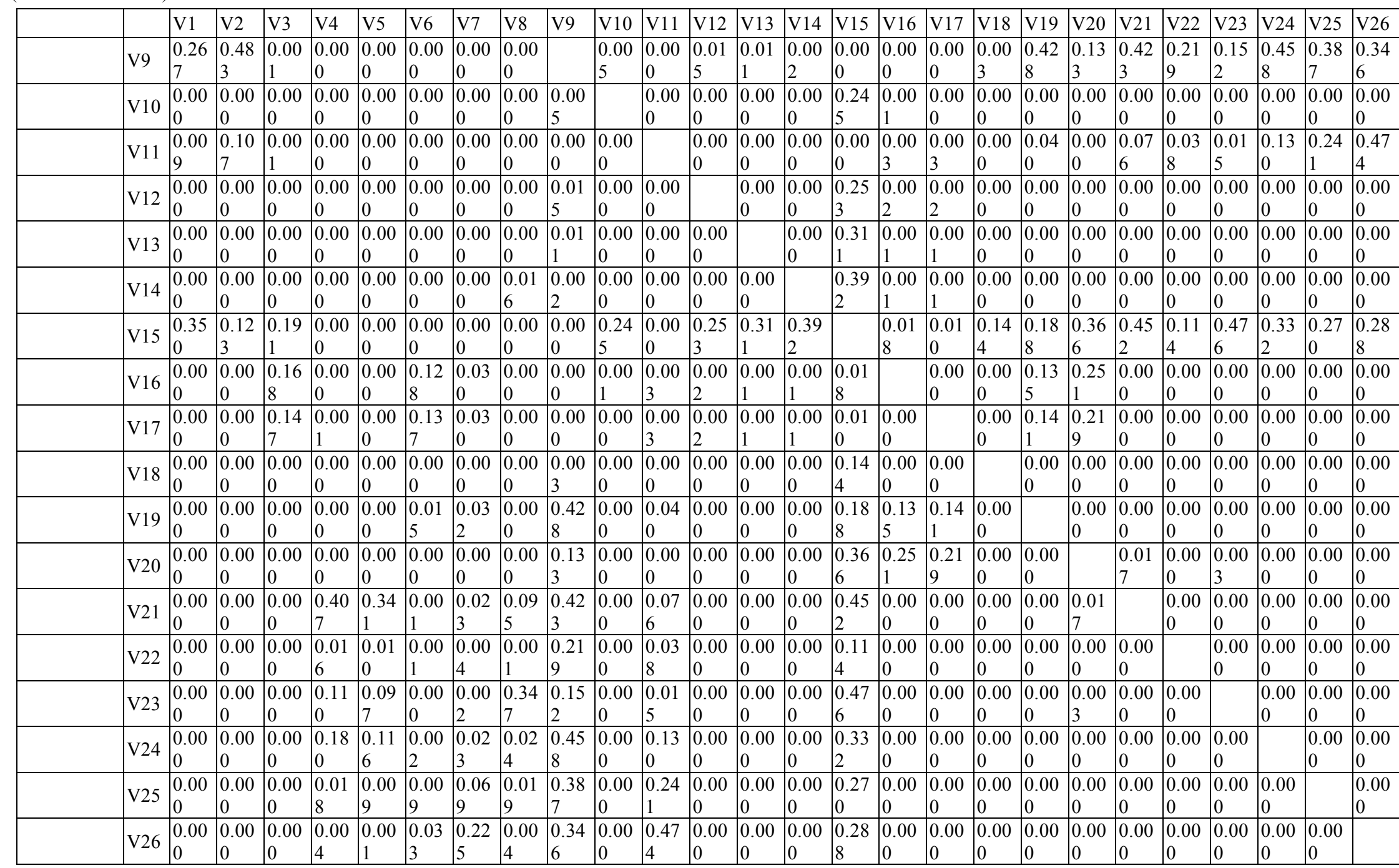

Note. Determinant $=1.844 \mathrm{E}-19$. 
Table 4

Inverse of Correlation Matrix

\begin{tabular}{|c|c|c|c|c|c|c|c|c|c|c|c|c|c|c|c|c|c|c|c|c|c|c|c|c|c|c|}
\hline & $V 1$ & V2 & V3 & V4 & V5 & $\sqrt{ } 6$ & $\sqrt{67}$ & V8 & 9 & V10 & V11 & 12 & V13 & V14 & 115 & V16 & $V 17$ & 18 & $\mathrm{~V} 19$ & 120 & 21 & V22 & V23 & V24 & V25 & V26 \\
\hline V1 & $\begin{array}{l}16.96 \\
1\end{array}$ & 0.148 & $\begin{array}{l}-0.67 \\
8\end{array}$ & 2.775 & $\begin{array}{l}-0.35 \\
3\end{array}$ & 299 & \begin{tabular}{|l|}
-0.42 \\
7
\end{tabular} & \begin{tabular}{|l}
-2.79 \\
4
\end{tabular} & 0.572 & $\begin{array}{l}-0.11 \\
4\end{array}$ & \begin{tabular}{|l}
-0.81 \\
5
\end{tabular} & 0.147 & \begin{tabular}{|l}
-3.82 \\
2
\end{tabular} & 1.700 & \begin{tabular}{|l}
-0.05 \\
2
\end{tabular} & 0.193 & & 3.962 & $\begin{array}{l}-0.56 \\
5\end{array}$ & 0.136 & 1.712 & \begin{tabular}{|l|}
-7.66 \\
6
\end{tabular} & \begin{tabular}{|l}
-3.79 \\
9
\end{tabular} & $\begin{array}{l}-5.08 \\
9\end{array}$ & \begin{tabular}{|l}
-0.64 \\
4
\end{tabular} & $\begin{array}{l}-1.25 \\
2\end{array}$ \\
\hline 2 & 148 & 5.444 & 0.015 & 1.817 & \begin{tabular}{|l|l}
0.35 \\
0 \\
\end{tabular} & \begin{tabular}{|l}
0.62 \\
9 \\
\end{tabular} & $0.276 \mid$ & \begin{tabular}{|l}
-0.67 \\
9 \\
\end{tabular} & 0.573 & 0.450 & \begin{tabular}{|l}
0.64 \\
5 \\
\end{tabular} & & 2.707 & 1.08 & 0.238 & \begin{tabular}{|l}
-0.06 \\
8 \\
\end{tabular} & -0.60 & 97 & \begin{tabular}{|l|l}
-0.33 \\
1 \\
\end{tabular} & \begin{tabular}{|l}
-2.62 \\
7 \\
\end{tabular} & .723 & \begin{tabular}{|l|l}
-0.94 \\
8 \\
\end{tabular} & \begin{tabular}{|l}
1.78 \\
6 \\
\end{tabular} & $\begin{array}{l}-1.06 \\
8 \\
\end{array}$ & .100 & \begin{tabular}{|l|}
-3.64 \\
0 \\
\end{tabular} \\
\hline V3 & .67 & 0.015 & 4.564 & \begin{tabular}{|l}
-0.06 \\
4
\end{tabular} & \begin{tabular}{|l}
-0.44 \\
1 \\
\end{tabular} & 0.237 & \begin{tabular}{|l|}
-0.62 \\
4
\end{tabular} & \begin{tabular}{|l}
-0.02 \\
3
\end{tabular} & 130 & $0.137 \mid$ & 0.765 & \begin{tabular}{|l|}
-0.58 \\
3
\end{tabular} & \begin{tabular}{|l}
-1.81 \\
8
\end{tabular} & 1.401 & \begin{tabular}{|l}
-0.17 \\
2
\end{tabular} & \begin{tabular}{|l}
-0.76 \\
9
\end{tabular} & 0.131 & \begin{tabular}{|l|}
-0.61 \\
9 \\
\end{tabular} & \begin{tabular}{|l}
-2.20 \\
4
\end{tabular} & \begin{tabular}{|l}
-0.08 \\
0
\end{tabular} & 305 & 0.638 & \begin{tabular}{|l}
-0.93 \\
6
\end{tabular} & 0.771 & \begin{tabular}{|l}
-0.08 \\
6
\end{tabular} & $\begin{array}{l}-0.09 \\
6\end{array}$ \\
\hline V4 & .775 & 1.817 & $\begin{array}{l}-0.06 \\
4\end{array}$ & $\begin{array}{l}13.20 \\
2\end{array}$ & $\begin{array}{l}-7.16 \\
7\end{array}$ & 0.867 & \begin{tabular}{|l|}
-0.59 \\
1
\end{tabular} & \begin{tabular}{|l|}
-2.77 \\
1
\end{tabular} & \begin{tabular}{|l}
-0.91 \\
7
\end{tabular} & 0.362 & \begin{tabular}{|l}
-2.64 \\
1
\end{tabular} & .829 & \begin{tabular}{|l}
-0.92 \\
1
\end{tabular} & $\begin{array}{l}30.43 \\
7\end{array}$ & \begin{tabular}{|l}
-0.52 \\
9
\end{tabular} & \begin{tabular}{|l}
-0.24 \\
8
\end{tabular} & $0.687 \mid$ & \begin{tabular}{|l|}
-3.57 \\
5
\end{tabular} & 0.043 & \begin{tabular}{|l}
-0.52 \\
2
\end{tabular} & \begin{tabular}{|l|}
-0.14 \\
1
\end{tabular} & \begin{tabular}{|l|}
-2.27 \\
2
\end{tabular} & \begin{tabular}{|l}
-2.38 \\
7
\end{tabular} & 0.402 & \begin{tabular}{|l}
-0.68 \\
1
\end{tabular} & 0.313 \\
\hline V5 & \begin{tabular}{|l}
-0.35 \\
3 \\
\end{tabular} & \begin{tabular}{|l}
-0.35 \\
0 \\
\end{tabular} & \begin{tabular}{|l}
-0.44 \\
1 \\
\end{tabular} & \begin{tabular}{|l|}
-7.16 \\
7 \\
\end{tabular} & $\begin{array}{l}10.33 \\
1 \\
\end{array}$ & 0.088 & \begin{tabular}{|l|}
-0.07 \\
0 \\
\end{tabular} & \begin{tabular}{|l}
-0.46 \\
3 \\
\end{tabular} & $\begin{array}{l}-0.71 \\
9 \\
\end{array}$ & \begin{tabular}{|l|}
-0.10 \\
8 \\
\end{tabular} & \begin{tabular}{|l}
-1.97 \\
2 \\
\end{tabular} & 0.417 & 9.644 & \begin{tabular}{|l|}
-1.62 \\
8 \\
\end{tabular} & \begin{tabular}{|l}
-0.51 \\
7 \\
\end{tabular} & \begin{tabular}{|l}
-0.45 \\
0 \\
\end{tabular} & 1.037 & \begin{tabular}{|l|}
-5.80 \\
9 \\
\end{tabular} & $\begin{array}{l}-2.90 \\
6 \\
\end{array}$ & 0.158 & \begin{tabular}{|l|}
-0.41 \\
0 \\
\end{tabular} & 0.291 & 1.389 & $\begin{array}{l}-0.15 \\
4 \\
\end{array}$ & 0.912 & -1.62 \\
\hline V6 & 299 & \begin{tabular}{|l}
-0.62 \\
9 \\
\end{tabular} & 0.237 & 0.86 & 0.088 & 3.719 & \begin{tabular}{|l|}
-3.06 \\
6 \\
\end{tabular} & \begin{tabular}{|l}
-1.47 \\
4 \\
\end{tabular} & $\begin{array}{l}-0.70 \\
6 \\
\end{array}$ & 0.08 & 0.89 & 0.102 & \begin{tabular}{|l}
-0.17 \\
7 \\
\end{tabular} & $0.131 \mid$ & \begin{tabular}{|l}
-0.14 \\
5 \\
\end{tabular} & \begin{tabular}{|l|}
-0.11 \\
1 \\
\end{tabular} & $0.502 \mid$ & \begin{tabular}{|l}
-0.60 \\
5 \\
\end{tabular} & 0.057 & \begin{tabular}{|l}
-0.43 \\
5 \\
\end{tabular} & .47 & \begin{tabular}{|l|}
-0.36 \\
5
\end{tabular} & \begin{tabular}{|l}
-0.34 \\
2 \\
\end{tabular} & 0.475 & \begin{tabular}{|l}
-0.27 \\
1 \\
\end{tabular} & 0.514 \\
\hline V7 & \begin{tabular}{|l}
-0.42 \\
7 \\
\end{tabular} & 276 & $\begin{array}{l}-0.62 \\
4 \\
\end{array}$ & \begin{tabular}{|l}
-0.59 \\
1 \\
\end{tabular} & \begin{tabular}{|l|}
-0.07 \\
0 \\
\end{tabular} & \begin{tabular}{|l}
-3.06 \\
6 \\
\end{tabular} & \begin{tabular}{|l|}
12.42 \\
5 \\
\end{tabular} & 1.39 & 0.366 & \begin{tabular}{|l|}
-0.66 \\
9 \\
\end{tabular} & \begin{tabular}{|l}
-9.85 \\
9 \\
\end{tabular} & \begin{tabular}{|l|}
-1.33 \\
3 \\
\end{tabular} & 2.073 & \begin{tabular}{|l|}
-0.25 \\
2 \\
\end{tabular} & 0.455 & 0.393 & \begin{tabular}{|l|}
-0.70 \\
7 \\
\end{tabular} & \begin{tabular}{|l}
-0.20 \\
3 \\
\end{tabular} & $\begin{array}{l}-0.00 \\
2 \\
\end{array}$ & 0.655 & 3 & 0.447 & 0.582 & $\begin{array}{l}-0.46 \\
6 \\
\end{array}$ & 6 & $\begin{array}{l}-0.62 \\
6 \\
\end{array}$ \\
\hline V8 & \begin{tabular}{|l|}
-2.79 \\
4 \\
\end{tabular} & \begin{tabular}{|l}
-0.67 \\
9 \\
\end{tabular} & \begin{tabular}{|l}
-0.02 \\
3 \\
\end{tabular} & \begin{tabular}{|l|}
-2.77 \\
1 \\
\end{tabular} & \begin{tabular}{|l|}
-0.46 \\
3 \\
\end{tabular} & \begin{tabular}{|l}
-1.47 \\
4 \\
\end{tabular} & 1.394 & 3.97 & 0.812 & 0.040 & \begin{tabular}{|l}
-0.35 \\
2 \\
\end{tabular} & \begin{tabular}{|l|}
-0.46 \\
8 \\
\end{tabular} & 0.732 & \begin{tabular}{|l|}
-0.53 \\
3 \\
\end{tabular} & 0.231 & \begin{tabular}{|l}
-0.18 \\
5 \\
\end{tabular} & \begin{tabular}{|l|}
-0.34 \\
6 \\
\end{tabular} & 0.666 & \begin{tabular}{|l|}
-0.01 \\
3 \\
\end{tabular} & \begin{tabular}{|l}
-0.47 \\
6 \\
\end{tabular} & $0.951 \mid$ & 1.402 & 1.311 & $\begin{array}{l}-0.30 \\
5 \\
\end{array}$ & \begin{tabular}{|l}
-0.11 \\
6 \\
\end{tabular} & 0.056 \\
\hline V9 & 572 & 0.573 & 1.130 & $\begin{array}{l}-0.91 \\
7\end{array}$ & \begin{tabular}{|l|}
-0.71 \\
9 \\
\end{tabular} & \begin{tabular}{|l}
-0.70 \\
6 \\
\end{tabular} & $0.366 \mid$ & 0.812 & 4.249 & $0.599 \mid$ & \begin{tabular}{|l}
0.24 \\
2 \\
\end{tabular} & \begin{tabular}{|l|}
-1.41 \\
0 \\
\end{tabular} & 0.734 & $0.304 \mid$ & \begin{tabular}{|l}
-0.09 \\
5 \\
\end{tabular} & \begin{tabular}{|l}
-0.43 \\
1 \\
\end{tabular} & \begin{tabular}{|l|}
-2.50 \\
8 \\
\end{tabular} & 0.637 & $\begin{array}{l}-1.15 \\
7 \\
\end{array}$ & $\begin{array}{l}-0.72 \\
5 \\
\end{array}$ & $0.017 \mid$ & 0.576 & 0.325 & $\begin{array}{l}-1.07 \\
7\end{array}$ & 0.503 & 7 \\
\hline 10 & \begin{tabular}{|l|}
-0.11 \\
4 \\
\end{tabular} & 450 & 0.137 & 0.362 & \begin{tabular}{|l}
-0.10 \\
8 \\
\end{tabular} & 0.086 & -0.66 & 0.040 & 0.599 & \begin{tabular}{|l|}
20.85 \\
0 \\
\end{tabular} & \begin{tabular}{|l}
-0.26 \\
5 \\
\end{tabular} & \begin{tabular}{|l}
-32.0 \\
62 \\
\end{tabular} & \begin{tabular}{|l}
13.64 \\
7 \\
\end{tabular} & 88 & 0.06 & 0.016 & 0.45 & \begin{tabular}{|l|}
-1.32 \\
0 \\
\end{tabular} & \begin{tabular}{|l}
-0.45 \\
8 \\
\end{tabular} & \begin{tabular}{|l}
-0.12 \\
2 \\
\end{tabular} & .37 & \begin{tabular}{|l|l|}
-0.23 \\
0 \\
\end{tabular} & \begin{tabular}{|l}
0.19 \\
3 \\
\end{tabular} & \begin{tabular}{|l}
-0.41 \\
0 \\
\end{tabular} & 0.230 & \begin{tabular}{|l}
-0.59 \\
7 \\
\end{tabular} \\
\hline 11 & \begin{tabular}{|l}
-0.81 \\
5
\end{tabular} & \begin{tabular}{|l}
-0.64 \\
5
\end{tabular} & 0.765 & \begin{tabular}{|l}
-2.64 \\
1 \\
\end{tabular} & \begin{tabular}{|l}
-1.97 \\
2
\end{tabular} & 0.893 & \begin{tabular}{|l|}
-9.85 \\
9
\end{tabular} & \begin{tabular}{|l}
-0.35 \\
2
\end{tabular} & \begin{tabular}{|l}
-0.24 \\
2
\end{tabular} & \begin{tabular}{|l|}
-0.26 \\
5
\end{tabular} & $\begin{array}{l}14.46 \\
9\end{array}$ & 1.473 & \begin{tabular}{|l}
-11.4 \\
29
\end{tabular} & \begin{tabular}{|l|}
-0.46 \\
0
\end{tabular} & \begin{tabular}{|l}
-0.35 \\
2
\end{tabular} & \begin{tabular}{|l|}
-0.08 \\
3
\end{tabular} & \begin{tabular}{|l|}
-0.48 \\
7
\end{tabular} & 6.412 & 3.726 & 0.247 & $0.166 \mid$ & 1.069 & \begin{tabular}{|l}
-0.61 \\
4
\end{tabular} & 0.385 & \begin{tabular}{|l}
-0.45 \\
5
\end{tabular} & $\begin{array}{l}10.10 \\
1\end{array}$ \\
\hline 12 & 0.147 & \begin{tabular}{|l}
0.41 \\
9 \\
\end{tabular} & \begin{tabular}{|l}
-0.58 \\
3 \\
\end{tabular} & 0.829 & 0.417 & 0.102 & \begin{tabular}{|l|}
-1.33 \\
3 \\
\end{tabular} & \begin{tabular}{|l|}
-0.46 \\
8 \\
\end{tabular} & \begin{tabular}{|l}
1.41 \\
0 \\
\end{tabular} & \begin{tabular}{|l|}
-32.0 \\
62 \\
\end{tabular} & 1.473 & $\begin{array}{l}74.80 \\
3 \\
\end{array}$ & \begin{tabular}{|l|}
-42.5 \\
77 \\
\end{tabular} & \begin{tabular}{|l|}
-0.02 \\
3 \\
\end{tabular} & \begin{tabular}{|l}
-0.43 \\
7 \\
\end{tabular} & 0.844 & \begin{tabular}{|l|}
-1.45 \\
6 \\
\end{tabular} & \begin{tabular}{|l|}
-2.44 \\
2 \\
\end{tabular} & 1.247 & 0.077 & \begin{tabular}{|l|}
-0.11 \\
4 \\
\end{tabular} & 0.222 & \begin{tabular}{|l}
-0.15 \\
8 \\
\end{tabular} & 0.422 & 0.068 & \begin{tabular}{|l}
-0.19 \\
7 \\
\end{tabular} \\
\hline 13 & \begin{tabular}{|l}
-3.82 \\
2 \\
\end{tabular} & 707 & $\begin{array}{l}-1.81 \\
8\end{array}$ & \begin{tabular}{|l}
-0.92 \\
1 \\
\end{tabular} & 9.644 & -0.17 & 2.07 & .732 & 0.734 & \begin{tabular}{|l|}
13.64 \\
7 \\
\end{tabular} & \begin{tabular}{|l}
-11.4 \\
29
\end{tabular} & \begin{tabular}{|l|}
-42.5 \\
77 \\
\end{tabular} & $\begin{array}{l}85.33 \\
8 \\
\end{array}$ & \begin{tabular}{|l|}
-8.63 \\
0 \\
\end{tabular} & 35 & 0.042 & 0.151 & \begin{tabular}{|l|}
-35.3 \\
24 \\
\end{tabular} & \begin{tabular}{|l|l|}
11.6 \\
72
\end{tabular} & $\begin{array}{l}-3.00 \\
4\end{array}$ & $\mid-3.31$ & .073 & 841 & $\begin{array}{l}-0.86 \\
9\end{array}$ & .81 & $\begin{array}{l}-5.34 \\
2\end{array}$ \\
\hline 14 & 1.700 & 1.089 & 1.401 & 3.437 & \begin{tabular}{|l|}
-1.62 \\
8 \\
\end{tabular} & 0.131 & \begin{tabular}{|l|}
-0.25 \\
2 \\
\end{tabular} & \begin{tabular}{|l|}
-0.53 \\
3 \\
\end{tabular} & 0.304 & 0.88 & \begin{tabular}{|l|l}
-0.46 \\
0 \\
\end{tabular} & $\begin{array}{l}-0.02 \\
3 \\
\end{array}$ & \begin{tabular}{|l}
-8.63 \\
0 \\
\end{tabular} & $7.617 \mid$ & \begin{tabular}{|l|}
-0.43 \\
4 \\
\end{tabular} & \begin{tabular}{|l|l|}
-0.38 \\
8 \\
\end{tabular} & 0.348 & 0.709 & \begin{tabular}{|l|l}
-0.42 \\
8 \\
\end{tabular} & \begin{tabular}{|l}
-0.74 \\
2 \\
\end{tabular} & $0.687 \mid$ & \begin{tabular}{|l|}
-0.68 \\
6 \\
\end{tabular} & \begin{tabular}{|l}
-2.48 \\
1 \\
\end{tabular} & \begin{tabular}{|l}
-0.87 \\
8 \\
\end{tabular} & \begin{tabular}{|l}
-0.21 \\
3 \\
\end{tabular} & 0.394 \\
\hline 15 & $\begin{array}{l}-0.05 \\
2 \\
\end{array}$ & 238 & $\begin{array}{l}-0.17 \\
2\end{array}$ & $\begin{array}{l}-0.52 \\
9 \\
\end{array}$ & \begin{tabular}{|l}
-0.51 \\
7 \\
\end{tabular} & \begin{tabular}{|l}
-0.14 \\
5 \\
\end{tabular} & 0.45 & 0.231 & \begin{tabular}{|l}
-0.09 \\
5 \\
\end{tabular} & 0.063 & \begin{tabular}{|l}
-0.35 \\
2 \\
\end{tabular} & \begin{tabular}{|l}
-0.43 \\
7 \\
\end{tabular} & 0.352 & \begin{tabular}{|l|}
-0.43 \\
4 \\
\end{tabular} & 1.486 & 1.360 & \begin{tabular}{|l|}
-1.20 \\
5 \\
\end{tabular} & 0.819 & 0.057 & \begin{tabular}{|l}
-0.03 \\
5 \\
\end{tabular} & 0.053 & \begin{tabular}{|l}
-0.43 \\
2 \\
\end{tabular} & 0.231 & $\begin{array}{l}-0.04 \\
2 \\
\end{array}$ & $\begin{array}{l}-0.10 \\
5 \\
\end{array}$ & 0.098 \\
\hline 16 & 0.193 & \begin{tabular}{|l}
-0.06 \\
8 \\
\end{tabular} & $\begin{array}{l}-0.76 \\
9 \\
\end{array}$ & \begin{tabular}{|l|}
-0.24 \\
8 \\
\end{tabular} & \begin{tabular}{|l|l}
-0.45 \\
0 \\
\end{tabular} & \begin{tabular}{|l}
-0.11 \\
1 \\
\end{tabular} & 0.39 & \begin{tabular}{|l|}
-0.18 \\
5 \\
\end{tabular} & \begin{tabular}{|l}
-0.43 \\
1 \\
\end{tabular} & $0.016 \mid$ & \begin{tabular}{|l}
-0.08 \\
3 \\
\end{tabular} & 0.844 & 0.042 & \begin{tabular}{|l|}
-0.38 \\
8 \\
\end{tabular} & 1.360 & \begin{tabular}{|l}
82.70 \\
6 \\
\end{tabular} & \begin{tabular}{|l|}
-81.9 \\
43 \\
\end{tabular} & \begin{tabular}{|l|}
-0.52 \\
0 \\
\end{tabular} & 0.821 & \begin{tabular}{|l}
-0.15 \\
5 \\
\end{tabular} & \begin{tabular}{|l|}
-0.03 \\
0 \\
\end{tabular} & \begin{tabular}{|l|}
-0.38 \\
9 \\
\end{tabular} & 0.109 & \begin{tabular}{|l}
-0.14 \\
1 \\
\end{tabular} & \begin{tabular}{|l}
-0.00 \\
3 \\
\end{tabular} & \begin{tabular}{|l}
-0.03 \\
9 \\
\end{tabular} \\
\hline 17 & \begin{tabular}{|l}
-0.84 \\
8 \\
\end{tabular} & \begin{tabular}{|l}
-0.60 \\
9 \\
\end{tabular} & 0.131 & 0.687 & 1.037 & 0.502 & \begin{tabular}{|l|}
-0.70 \\
7 \\
\end{tabular} & \begin{tabular}{|l}
-0.34 \\
6 \\
\end{tabular} & -2.50 & 0.45 & \begin{tabular}{|l}
-0.48 \\
7 \\
\end{tabular} & \begin{tabular}{|l}
1.45 \\
6 \\
\end{tabular} & 0.151 & 0.34 & \begin{tabular}{|l}
-1.20 \\
5 \\
\end{tabular} & \begin{tabular}{|l|}
-81.9 \\
43 \\
\end{tabular} & \begin{tabular}{|l|}
44.92 \\
4 \\
\end{tabular} & 1. & \begin{tabular}{|l}
-0.89 \\
3 \\
\end{tabular} & 0.803 & 0.022 & \begin{tabular}{|l|}
-0.32 \\
4 \\
\end{tabular} & \begin{tabular}{|l}
-0.32 \\
6 \\
\end{tabular} & 0.879 & \begin{tabular}{|l}
-0.49 \\
4 \\
\end{tabular} & 0.661 \\
\hline 18 & 3.962 & \begin{tabular}{|l|}
1.97 \\
0 \\
\end{tabular} & \begin{tabular}{|l}
-0.61 \\
9 \\
\end{tabular} & \begin{tabular}{|l}
-3.57 \\
5 \\
\end{tabular} & \begin{tabular}{|l}
5.80 \\
9 \\
\end{tabular} & -0.60 & \begin{tabular}{|l|}
-0.20 \\
3 \\
\end{tabular} & 0.66 & 637 & \begin{tabular}{|l|}
-1.32 \\
0 \\
\end{tabular} & 6.41 & \begin{tabular}{|l}
2.44 \\
2 \\
\end{tabular} & \begin{tabular}{|l}
-35.3 \\
24 \\
\end{tabular} & 0.70 & 0.819 & \begin{tabular}{|l}
-0.52 \\
0 \\
\end{tabular} & 1.405 & 34.90 & 6.277 & 1.739 & 785 & \begin{tabular}{|l|}
-2.89 \\
7 \\
\end{tabular} & -2.65 & \begin{tabular}{|l}
-0.46 \\
6 \\
\end{tabular} & $\mid-1.44$ & 2.65 \\
\hline
\end{tabular}


(Table 4 continued)

\begin{tabular}{|c|c|c|c|c|c|c|c|c|c|c|c|c|c|c|c|c|c|c|c|c|c|c|c|c|c|c|}
\hline & & & & & & & & & & & & & & & & & & & & & & & & & & \\
\hline 19 & & $\begin{array}{l}-0.53 \\
1\end{array}$ & 4 & 043 & $\begin{array}{l}-2 \\
6\end{array}$ & 057 & & & $\begin{array}{l}-1.15 \\
7\end{array}$ & & 5 & & -1 & & & & & & & & & 1 & & 0 & & \\
\hline & 136 & & $\begin{array}{l}-0 \\
0\end{array}$ & & 158 & & 655 & & & & & & & & & & & & & & & & 7 & & & \\
\hline 21 & 712 & 723 & 0.305 & & & & 153 & 951 & $01^{\prime}$ & 0.37 & 66 & & & 687 & 0.053 & & 022 & & & & 982 & 227 & & & 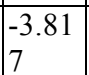 & .0 \\
\hline 22 & & & 638 & \begin{tabular}{|l|}
-2.27 \\
2
\end{tabular} & 291 & & 447 & 40 & 0.576 & & 069 & 222 & 2.07 & & & & & & 26 & & 227 & & .10 & 2 & 7 & \\
\hline 3 & & $\begin{array}{l}-1.78 \\
6\end{array}$ & - & & 385 & & 582 & 311 & 325 & -0.19 & & & 5.84 & 48 & 0.231 & .10 & & & & 007 & 7 & & .11 & .843 & & 6 \\
\hline 4 & -5.08 & $\begin{array}{l}-1.06 \\
8\end{array}$ & 71 & 0.402 & & .475 & & & & \begin{tabular}{|l|}
-0.41 \\
0
\end{tabular} & 385 & 0.422 & & & & & 879 & & 90 & 92 & \begin{tabular}{|l|}
-4.18 \\
5
\end{tabular} & 2 & 43 & t5 & & \\
\hline & \begin{tabular}{|l|}
-0.64 \\
\end{tabular} & 00 & 88 & & 0 & & 0.698 & & 0.503 & 0 & & & 2 & -0.21 & -0.10 & \begin{tabular}{|l|}
-0.00 \\
\end{tabular} & 9 & & 5 & 9 & -3.81 & 87 & 16 & - & 22. & s. \\
\hline & & 64 & $\begin{array}{l}-0.09 \\
6\end{array}$ & 313 & & 0.514 & 52 & 0.05 & & & 01 & 7 & 2 & & 000 & & $0 \in 6$ & & 0.7 & $2.052 \mid$ & 0.0 & & 1.674 & 1.15 & 8 & \\
\hline
\end{tabular}

Table 5

KMO and Bartlett's Test

Kaiser-Meyer-Olkin measure of sampling adequacy

Bartlett's test of sphericity

\begin{tabular}{|l|l|} 
& 0.896 \\
\hline Approx. Chi-Square & 16154.888 \\
\hline df & 325 \\
\hline Sig. & 0.000
\end{tabular}

Table 6

Communalities

\begin{tabular}{|l|l|l|}
\hline & Initial & Extraction \\
\hline V1 & 1.000 & 0.911 \\
\hline V2 & 1.000 & 0.870 \\
\hline V3 & 1.000 & 0.662 \\
\hline V4 & 1.000 & 0.879 \\
\hline V5 & 1.000 & 0.843 \\
\hline V6 & 1.000 & 0.711 \\
\hline V7 & 1.000 & 0.793 \\
\hline
\end{tabular}


(Table 6 continued)

\begin{tabular}{|l|l|l|}
\hline & Initial & Extraction \\
\hline V9 & 1.000 & 0.824 \\
\hline V10 & 1.000 & 0.885 \\
\hline V11 & 1.000 & 0.822 \\
\hline V12 & 1.000 & 0.964 \\
\hline V13 & 1.000 & 0.975 \\
\hline V14 & 1.000 & 0.824 \\
\hline V15 & 1.000 & 0.480 \\
\hline V16 & 1.000 & 0.953 \\
\hline V17 & 1.000 & 0.956 \\
\hline V18 & 1.000 & 0.938 \\
\hline V19 & 1.000 & 0.729 \\
\hline V20 & 1.000 \\
\hline V21 & 0.807 \\
\hline V22 & 1.000 \\
\hline V23 & 1.000 & 0.794 \\
\hline V24 & 1.000 & 0.883 \\
\hline V25 & 1.000 & 0.844 \\
\hline V26 & 1.000 & 0.866 \\
\hline
\end{tabular}

Note. Extraction Method: Principal Component Analysis.

Table 7

Total Variance

\begin{tabular}{|c|c|c|c|c|c|c|c|c|c|}
\hline & Initial eigenvalues & & & \begin{tabular}{|l|}
$\begin{array}{l}\text { Extraction sums of } \\
\text { squared loadings }\end{array}$ \\
\end{tabular} & & & $\begin{array}{l}\text { Rotation sums of } \\
\text { squared loadings }\end{array}$ & & \\
\hline Component & Total & $\%$ of variance & Cumulative \% & Total & $\begin{array}{l}\% \text { of } \\
\text { variance }\end{array}$ & Cumulative \% & Total & $\%$ of variance & Cumulative \% \\
\hline 1 & 9.721 & 37.389 & 37.389 & 9.721 & 37.389 & 37.389 & 6.920 & 26.617 & 26.617 \\
\hline 2 & 5.538 & 21.298 & 58.687 & 5.538 & 21.298 & 58.687 & 6.224 & 23.937 & 50.554 \\
\hline 3 & 3.835 & 14.752 & 73.439 & 3.835 & 14.752 & 73.439 & 4.286 & 16.485 & 67.039 \\
\hline 4 & 1.407 & 5.410 & 78.849 & 1.407 & 5.410 & 78.849 & 2.661 & 10.233 & 77.273 \\
\hline 5 & 1.244 & 4.784 & 83.633 & 1.244 & 4.784 & 83.633 & 1.654 & 6.360 & 83.633 \\
\hline 6 & 0.953 & 3.664 & 87.296 & & & & & & \\
\hline
\end{tabular}


(Table 7 continued)

\begin{tabular}{|c|c|c|c|c|c|c|c|c|c|}
\hline & Initial eigenvalues & & & $\begin{array}{l}\text { Extraction sums of } \\
\text { squared loadings }\end{array}$ & & & \begin{tabular}{|l|} 
Rotation sums of \\
squared loadings
\end{tabular} & & \\
\hline Component & Total & $\%$ of variance & Cumulative \% & Total & $\begin{array}{l}\% \text { of } \\
\text { variance }\end{array}$ & Cumulative \% & Total & $\%$ of variance & Cumulative \% \\
\hline 7 & 0.670 & 2.576 & 89.872 & & & & & & \\
\hline 8 & 0.429 & 1.650 & 91.522 & & & & & & \\
\hline 9 & 0.352 & 1.355 & 92.878 & & & & & & \\
\hline 10 & 0.324 & 1.244 & 94.122 & & & & & & \\
\hline 11 & 0.281 & 1.081 & 95.203 & & & & & & \\
\hline 12 & 0.223 & 0.857 & 96.060 & & & & & & \\
\hline 13 & 0.180 & 0.692 & 96.752 & & & & & & \\
\hline 14 & 0.168 & 0.648 & 97.400 & & & & & & \\
\hline 15 & 0.138 & 0.530 & 97.930 & & & & & & \\
\hline 16 & 0.116 & 0.445 & 98.374 & & & & & & \\
\hline 17 & 0.111 & 0.427 & 98.802 & & & & & & \\
\hline 18 & $7.842 \mathrm{E}-02$ & 0.302 & 99.103 & & & & & & \\
\hline 19 & $5.153 \mathrm{E}-02$ & 0.198 & 99.301 & & & & & & \\
\hline 20 & $4.869 \mathrm{E}-02$ & 0.187 & 99.489 & & & & & & \\
\hline 21 & $4.418 \mathrm{E}-02$ & 0.170 & 99.659 & & & & & & \\
\hline 22 & $3.624 \mathrm{E}-02$ & 0.139 & 99.798 & & & & & & \\
\hline 23 & 2.482E-02 & $9.546 \mathrm{E}-02$ & 99.893 & & & & & & \\
\hline 24 & $1.461 \mathrm{E}-02$ & $5.618 \mathrm{E}-02$ & 99.950 & & & & & & \\
\hline 25 & $7.076 \mathrm{E}-03$ & $2.721 \mathrm{E}-02$ & 99.977 & & & & & & \\
\hline 26 & $6.028 \mathrm{E}-03$ & $2.318 \mathrm{E}-02$ & 100.000 & & & & & & \\
\hline
\end{tabular}

Note. Extraction Method: Principal Component Analysis. 
Table 8

Component Matrix ${ }^{a}$

\begin{tabular}{|c|c|c|c|c|c|}
\hline & Component & & & & \\
\hline & 1 & 2 & 3 & 4 & 5 \\
\hline \begin{tabular}{|l} 
V1 \\
\end{tabular} & 0.722 & -0.561 & 0.229 & -0.138 & $6.071 \mathrm{E}-02$ \\
\hline \begin{tabular}{|l|}
$\mathrm{V} 2$ \\
\end{tabular} & 0.657 & -0.359 & 0.195 & $-2.033 \mathrm{E}-02$ & 0.521 \\
\hline V3 & 0.750 & $9.756 \mathrm{E}-02$ & -0.273 & 0.102 & $-7.032 \mathrm{E}-02$ \\
\hline \begin{tabular}{|l|} 
V4 \\
\end{tabular} & 0.418 & 0.666 & 0.392 & -0.319 & $-7.050 \mathrm{E}-02$ \\
\hline \begin{tabular}{|l|}
$\mathrm{V} 5$ \\
\end{tabular} & 0.411 & 0.626 & 0.412 & -0.328 & $-6.228 \mathrm{E}-02$ \\
\hline \begin{tabular}{|l|}
$\mathrm{V} 6$ \\
\end{tabular} & 0.246 & 0.743 & 0.162 & $4.954 \mathrm{E}-02$ & 0.264 \\
\hline \begin{tabular}{|l|}
$\mathrm{V7}$ \\
\end{tabular} & 0.292 & 0.800 & 0.198 & $-2.293 \mathrm{E}-02$ & -0.165 \\
\hline \begin{tabular}{|l|}
$\mathrm{V} 8$ \\
\end{tabular} & 0.357 & 0.480 & 0.384 & -0.267 & 0.474 \\
\hline V9 & $2.496 \mathrm{E}-02$ & 0.262 & 0.778 & 0.374 & -0.101 \\
\hline \begin{tabular}{|l|} 
V10 \\
\end{tabular} & 0.788 & 0.315 & -0.372 & 0.123 & -0.104 \\
\hline \begin{tabular}{|l|} 
V11 \\
\end{tabular} & 0.319 & 0.785 & 0.269 & \begin{tabular}{|l}
$7.593 \mathrm{E}-02$ \\
\end{tabular} & -0.159 \\
\hline \begin{tabular}{|l|} 
V12 \\
\end{tabular} & 0.827 & 0.323 & -0.373 & 0.168 & $-9.626 \mathrm{E}-02$ \\
\hline V13 & 0.831 & 0.313 & -0.383 & 0.177 & $-8.869 \mathrm{E}-02$ \\
\hline \begin{tabular}{|l|} 
V14 \\
\end{tabular} & 0.755 & 0.191 & -0.403 & 0.215 & $-9.198 \mathrm{E}-02$ \\
\hline \begin{tabular}{|l|} 
V15 \\
\end{tabular} & 0.119 & 0.308 & 0.344 & -0.433 & -0.255 \\
\hline \begin{tabular}{|l|} 
V16 \\
\end{tabular} & 0.133 & -0.114 & 0.835 & 0.469 & $-6.787 \mathrm{E}-02$ \\
\hline \begin{tabular}{|l|} 
V17 \\
\end{tabular} & 0.130 & -0.114 & 0.838 & 0.466 & $-7.496 \mathrm{E}-02$ \\
\hline \begin{tabular}{|l|} 
V18 \\
\end{tabular} & 0.807 & 0.368 & -0.376 & $6.902 \mathrm{E}-02$ & $-6.804 \mathrm{E}-02$ \\
\hline \begin{tabular}{|l|} 
V19 \\
\end{tabular} & 0.793 & $2.884 \mathrm{E}-02$ & -0.193 & 0.242 & $-6.551 \mathrm{E}-02$ \\
\hline \begin{tabular}{|l|} 
V20 \\
\end{tabular} & 0.509 & 0.277 & -0.119 & 0.196 & 0.647 \\
\hline \begin{tabular}{|l|}
$\mathrm{V} 21$ \\
\end{tabular} & 0.686 & -0.515 & 0.133 & \begin{tabular}{|l|}
$-5.779 \mathrm{E}-02$ \\
\end{tabular} & -0.196 \\
\hline \begin{tabular}{|l|}
$\mathrm{V} 22$ \\
\end{tabular} & 0.751 & -0.501 & 0.196 & -0.163 & $5.180 \mathrm{E}-02$ \\
\hline V23 & 0.736 & -0.522 & 0.131 & $-7.300 \mathrm{E}-02$ & $-8.462 \mathrm{E}-02$ \\
\hline \begin{tabular}{|l}
$\mathrm{V} 24$ \\
\end{tabular} & 0.734 & -0.520 & 0.197 & -0.134 & $-1.730 \mathrm{E}-02$ \\
\hline V25 & 0.771 & -0.475 & 0.162 & -0.154 & $-7.347 \mathrm{E}-02$ \\
\hline \begin{tabular}{|l|} 
V26 \\
\end{tabular} & 0.788 & -0.431 & 0.143 & -0.154 & $-6.746 \mathrm{E}-02$ \\
\hline
\end{tabular}

Note. Extraction Method: Principal Component Analysis, 5 components extracted. 
Table 9

Reproduced Correlations

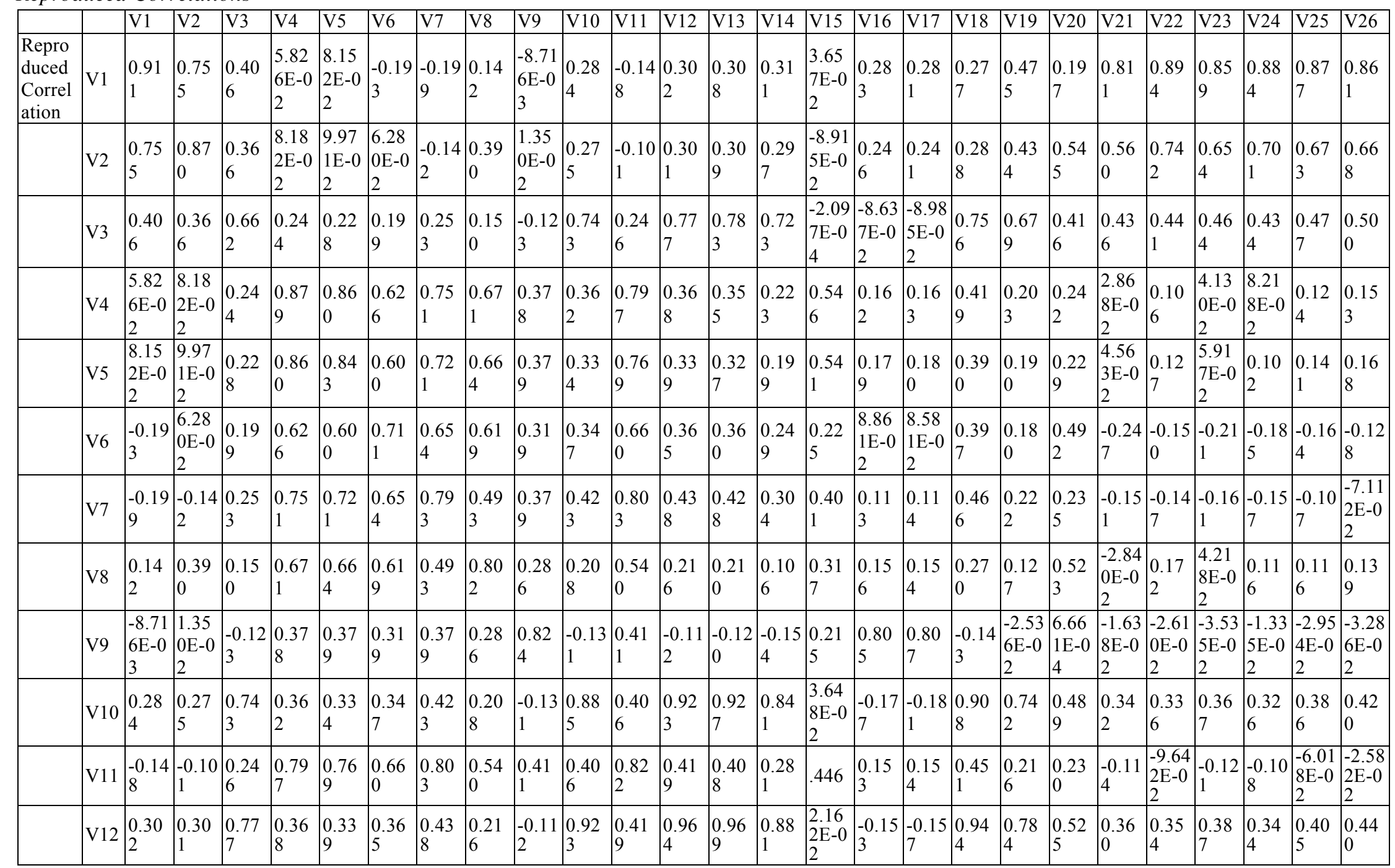




\begin{tabular}{|c|c|c|c|c|c|c|c|c|c|c|c|c|c|c|c|c|c|c|c|c|c|c|c|c|c|c|}
\hline & V1 & V2 & V3 & V4 & V5 & V6 & V7 & V8 & V9 & V10 & V11 & V12 & V13 & V14 & V15 & V16 & V17 & V18 & V19 & V20 & V21 & V22 & V23 & V24 & V25 & V26 \\
\hline V13 & $\begin{array}{l}0.30 \\
8\end{array}$ & $\begin{array}{l}0.30 \\
9\end{array}$ & $\begin{array}{l}0.78 \\
3\end{array}$ & $\begin{array}{l}0.35 \\
5\end{array}$ & $\begin{array}{l}0.32 \\
7\end{array}$ & $\begin{array}{l}0.36 \\
0\end{array}$ & $\begin{array}{l}0.42 \\
8\end{array}$ & $\begin{array}{l}0.21 \\
0\end{array}$ & \begin{tabular}{|l|}
-0.12 \\
0
\end{tabular} & $\begin{array}{l}0.92 \\
7\end{array}$ & $\begin{array}{l}0.40 \\
8\end{array}$ & $\begin{array}{l}0.96 \\
9\end{array}$ & $\begin{array}{l}0.97 \\
5\end{array}$ & \begin{tabular}{|l|}
0.88 \\
8
\end{tabular} & \begin{tabular}{|l|}
9.66 \\
$0 \mathrm{E}-0$ \\
3 \\
\end{tabular} & $\left|\begin{array}{l}-0.15 \\
6\end{array}\right|$ & $\mid \begin{array}{l}-0.16 \\
0\end{array}$ & $\mid \begin{array}{l}0.94 \\
8\end{array}$ & $\left|\begin{array}{l}0.79 \\
1\end{array}\right|$ & $\begin{array}{l}0.53 \\
2\end{array}$ & $\begin{array}{l}0.36 \\
5\end{array}$ & $\begin{array}{l}0.35 \\
9\end{array}$ & $\begin{array}{l}0.39 \\
3\end{array}$ & $\begin{array}{l}0.35 \\
0\end{array}$ & $\begin{array}{l}0.41 \\
0\end{array}$ & $\begin{array}{l}0.44 \\
5\end{array}$ \\
\hline V14 & $\begin{array}{l}0.31 \\
1\end{array}$ & $\begin{array}{l}0.29 \\
7\end{array}$ & $\begin{array}{l}0.72 \\
3\end{array}$ & $\begin{array}{l}0.22 \\
3\end{array}$ & $\begin{array}{l}0.19 \\
9\end{array}$ & $\begin{array}{l}0.24 \\
9\end{array}$ & $\begin{array}{l}0.30 \\
4\end{array}$ & $\begin{array}{l}0.10 \\
6\end{array}$ & $\begin{array}{l}-0.15 \\
4\end{array}$ & $\begin{array}{l}0.84 \\
1\end{array}$ & $\begin{array}{l}0.28 \\
1\end{array}$ & $\begin{array}{l}0.88 \\
1\end{array}$ & $\begin{array}{l}0.88 \\
8\end{array}$ & $\begin{array}{l}0.82 \\
4\end{array}$ & $\begin{array}{l}-5.92 \\
3 \mathrm{E}-0 \\
2\end{array}$ & $\begin{array}{l}-0.15 \\
0\end{array}$ & $\mid \begin{array}{l}-0.15 \\
5\end{array}$ & $\begin{array}{l}0.85 \\
2\end{array}$ & $\left|\begin{array}{l}0.74 \\
0\end{array}\right|$ & $\begin{array}{l}0.46 \\
8\end{array}$ & $\begin{array}{l}0.37 \\
2\end{array}$ & $\begin{array}{l}0.35 \\
3\end{array}$ & $\begin{array}{l}0.39 \\
6\end{array}$ & $\begin{array}{l}0.34 \\
8\end{array}$ & $\begin{array}{l}0.40 \\
0\end{array}$ & $\begin{array}{l}0.42 \\
9\end{array}$ \\
\hline V15 & $\begin{array}{l}3.65 \\
7 \mathrm{E}-0 \\
2\end{array}$ & \begin{tabular}{|l|}
-8.91 \\
$5 \mathrm{E}-0$ \\
2 \\
\end{tabular} & $\begin{array}{l}-2.09 \\
7 \mathrm{E}-0 \\
4\end{array}$ & $\begin{array}{l}0.54 \\
6\end{array}$ & $\begin{array}{l}0.54 \\
1\end{array}$ & \begin{tabular}{l|}
0.22 \\
5
\end{tabular} & $\begin{array}{l}0.40 \\
1\end{array}$ & $\begin{array}{l}0.31 \\
7\end{array}$ & $\begin{array}{l}0.21 \\
5\end{array}$ & \begin{tabular}{|l|}
3.64 \\
$8 \mathrm{E}-0$ \\
2 \\
\end{tabular} & $\begin{array}{l}0.44 \\
6\end{array}$ & $\begin{array}{l}2.16 \\
2 \mathrm{E}-0 \\
2\end{array}$ & \begin{tabular}{|l}
9.66 \\
$0 \mathrm{E}-0$ \\
3 \\
\end{tabular} & \begin{tabular}{|l|}
5.92 \\
$3 \mathrm{E}-0$ \\
2 \\
\end{tabular} & $\begin{array}{l}0.48 \\
0\end{array}$ & \begin{tabular}{|l|}
8.20 \\
$2 \mathrm{E}-0$ \\
2
\end{tabular} & \begin{tabular}{|l|l}
8.57 \\
$7 \mathrm{E}-0$ \\
2
\end{tabular} & \begin{tabular}{|l|}
6.82 \\
$5 \mathrm{E}-0$ \\
2 \\
\end{tabular} & \begin{tabular}{|l}
-5.05 \\
$9 \mathrm{E}-0$ \\
2 \\
\end{tabular} & \begin{tabular}{|l|}
-0.14 \\
5
\end{tabular} & \begin{tabular}{|l|}
4.41 \\
$6 \mathrm{E}-0$ \\
2
\end{tabular} & \begin{tabular}{|l|}
6.06 \\
$0 \mathrm{E}-0$ \\
2 \\
\end{tabular} & \begin{tabular}{|l|}
2.56 \\
$1 \mathrm{E}-0$ \\
2
\end{tabular} & \begin{tabular}{|l|}
5.79 \\
$7 \mathrm{E}-0$ \\
2
\end{tabular} & \begin{tabular}{|l}
8.68 \\
$9 \mathrm{E}-0$ \\
2 \\
\end{tabular} & \begin{tabular}{|l|}
9.46 \\
$0 \mathrm{E}-0$ \\
2 \\
\end{tabular} \\
\hline V16 & $\begin{array}{l}0.28 \\
3\end{array}$ & $\begin{array}{l}0.24 \\
6\end{array}$ & \begin{tabular}{|l}
-8.63 \\
$7 \mathrm{E}-0$ \\
2 \\
\end{tabular} & $\begin{array}{l}0.16 \\
2\end{array}$ & $\begin{array}{l}0.17 \\
9\end{array}$ & \begin{tabular}{|l|}
8.86 \\
$1 \mathrm{E}-0$ \\
2 \\
\end{tabular} & $\begin{array}{l}0.11 \\
3\end{array}$ & $\begin{array}{l}0.15 \\
6\end{array}$ & $\begin{array}{l}0.80 \\
5\end{array}$ & \begin{tabular}{|l}
-0.17 \\
7
\end{tabular} & $\begin{array}{l}0.15 \\
3\end{array}$ & $\begin{array}{l}-0.15 \\
3\end{array}$ & $\begin{array}{l}-0.15 \\
6\end{array}$ & \begin{tabular}{|l|}
-0.15 \\
0
\end{tabular} & \begin{tabular}{|l|}
8.20 \\
$2 \mathrm{E}-0$ \\
2 \\
\end{tabular} & $\begin{array}{l}0.95 \\
3\end{array}$ & $\left|\begin{array}{l}0.95 \\
4\end{array}\right|$ & \begin{tabular}{|l|}
-0.21 \\
1
\end{tabular} & \begin{tabular}{|l|}
5.93 \\
$9 \mathrm{E}-0$ \\
2 \\
\end{tabular} & \begin{tabular}{|l|}
1.53 \\
$5 \mathrm{E}-0$ \\
2 \\
\end{tabular} & $\begin{array}{l}0.24 \\
7\end{array}$ & $\begin{array}{l}0.24 \\
1\end{array}$ & $\begin{array}{l}0.23 \\
9\end{array}$ & $\begin{array}{l}0.26 \\
0\end{array}$ & $\begin{array}{l}0.22 \\
5\end{array}$ & $\begin{array}{l}0.20 \\
6\end{array}$ \\
\hline V17 & $\begin{array}{l}0.28 \\
1\end{array}$ & $\begin{array}{l}0.24 \\
1\end{array}$ & \begin{tabular}{|l}
-8.98 \\
$5 \mathrm{E}-0$ \\
2 \\
\end{tabular} & $\begin{array}{l}0.16 \\
3\end{array}$ & $\begin{array}{l}0.18 \\
0\end{array}$ & \begin{tabular}{|l|}
8.58 \\
$1 \mathrm{E}-0$ \\
2 \\
\end{tabular} & $\begin{array}{l}0.11 \\
4\end{array}$ & $\begin{array}{l}0.15 \\
4\end{array}$ & $\begin{array}{l}0.80 \\
7\end{array}$ & $\begin{array}{l}-0.18 \\
1\end{array}$ & $\begin{array}{l}0.15 \\
4\end{array}$ & $\begin{array}{l}-0.15 \\
7\end{array}$ & $\begin{array}{l}-0.16 \\
0\end{array}$ & $\begin{array}{l}-0.15 \\
5\end{array}$ & \begin{tabular}{|l|}
8.57 \\
$7 \mathrm{E}-0$ \\
2 \\
\end{tabular} & $\begin{array}{l}0.95 \\
4\end{array}$ & $\begin{array}{l}0.95 \\
6\end{array}$ & \begin{tabular}{|l|}
-0.21 \\
5
\end{tabular} & \begin{tabular}{|l|}
5.55 \\
$1 \mathrm{E}-0$ \\
2 \\
\end{tabular} & \begin{tabular}{|l|}
2.29 \\
$7 \mathrm{E}-0$ \\
2 \\
\end{tabular} & $\begin{array}{l}0.24 \\
7\end{array}$ & $\begin{array}{l}0.23 \\
9\end{array}$ & $\begin{array}{l}0.23 \\
7\end{array}$ & $\begin{array}{l}0.25 \\
9\end{array}$ & $\begin{array}{l}0.22 \\
4\end{array}$ & $\begin{array}{l}0.20 \\
5\end{array}$ \\
\hline V18 & $\begin{array}{l}0.27 \\
7\end{array}$ & $\begin{array}{l}0.28 \\
8\end{array}$ & .756 & $\begin{array}{l}0.41 \\
9\end{array}$ & $\begin{array}{l}0.39 \\
0\end{array}$ & $\begin{array}{l}0.39 \\
7\end{array}$ & $\begin{array}{l}0.46 \\
6\end{array}$ & $\begin{array}{l}0.27 \\
0\end{array}$ & \begin{tabular}{|l|}
-0.14 \\
3
\end{tabular} & $\begin{array}{l}0.90 \\
8\end{array}$ & $\begin{array}{l}0.45 \\
1\end{array}$ & $\begin{array}{l}0.94 \\
4\end{array}$ & $\begin{array}{l}0.94 \\
8\end{array}$ & $\begin{array}{l}0.85 \\
2\end{array}$ & \begin{tabular}{|l|}
6.82 \\
$5 \mathrm{E}-0$ \\
2 \\
\end{tabular} & $\left|\begin{array}{l}-0.21 \\
1\end{array}\right|$ & $\mid \begin{array}{l}-0.21 \\
5\end{array}$ & $\begin{array}{l}0.93 \\
8\end{array}$ & $\begin{array}{l}0.74 \\
4\end{array}$ & $\begin{array}{l}0.52 \\
7\end{array}$ & $\begin{array}{l}0.32 \\
3\end{array}$ & $\begin{array}{l}0.33 \\
4\end{array}$ & $\begin{array}{l}0.35 \\
4\end{array}$ & $\begin{array}{l}0.31 \\
9\end{array}$ & $\begin{array}{l}0.38 \\
1\end{array}$ & $\begin{array}{l}0.41 \\
8\end{array}$ \\
\hline V19 & $\begin{array}{l}0.47 \\
5\end{array}$ & $\begin{array}{l}0.43 \\
4\end{array}$ & $\begin{array}{l}0.67 \\
9\end{array}$ & $\begin{array}{l}0.20 \\
3\end{array}$ & $\begin{array}{l}0.19 \\
0\end{array}$ & $\begin{array}{l}0.18 \\
0\end{array}$ & $\begin{array}{l}0.22 \\
2\end{array}$ & $\begin{array}{l}0.12 \\
7\end{array}$ & \begin{tabular}{|l|}
-2.53 \\
$6 \mathrm{E}-0$ \\
2 \\
\end{tabular} & $\begin{array}{l}0.74 \\
2\end{array}$ & $\begin{array}{l}0.21 \\
6\end{array}$ & $\begin{array}{l}0.78 \\
4\end{array}$ & $\begin{array}{l}0.79 \\
1\end{array}$ & \begin{tabular}{|l|}
0.74 \\
0
\end{tabular} & \begin{tabular}{|l|}
-5.05 \\
$9 \mathrm{E}-0$ \\
2 \\
\end{tabular} & \begin{tabular}{|l|}
5.93 \\
$9 \mathrm{E}-0$ \\
2 \\
\end{tabular} & \begin{tabular}{|l|}
5.55 \\
$1 \mathrm{E}-0$ \\
2 \\
\end{tabular} & $\begin{array}{l}0.74 \\
4\end{array}$ & $\begin{array}{l}0.72 \\
9\end{array}$ & \begin{tabular}{l|}
0.43 \\
9
\end{tabular} & $\begin{array}{l}0.50 \\
2\end{array}$ & $\begin{array}{l}0.50 \\
1\end{array}$ & $\begin{array}{l}0.53 \\
1\end{array}$ & $\begin{array}{l}0.49 \\
8\end{array}$ & $\begin{array}{l}0.53 \\
4\end{array}$ & $\begin{array}{l}0.55 \\
2\end{array}$ \\
\hline V20 & $\begin{array}{l}0.19 \\
7\end{array}$ & $\begin{array}{l}0.54 \\
5\end{array}$ & $\begin{array}{l}0.41 \\
6\end{array}$ & $\begin{array}{l}0.24 \\
2\end{array}$ & $\begin{array}{l}0.22 \\
9\end{array}$ & $\begin{array}{l}0.49 \\
2\end{array}$ & $\begin{array}{l}0.23 \\
5\end{array}$ & $\begin{array}{l}0.52 \\
3\end{array}$ & \begin{tabular}{|l|}
6.66 \\
$1 \mathrm{E}-0$ \\
4 \\
\end{tabular} & $\begin{array}{l}0.48 \\
9\end{array}$ & $\begin{array}{l}0.23 \\
0\end{array}$ & $\begin{array}{l}0.52 \\
5\end{array}$ & $\begin{array}{l}0.53 \\
2\end{array}$ & \begin{tabular}{|l|}
0.46 \\
8
\end{tabular} & \begin{tabular}{|l|}
-0.14 \\
5
\end{tabular} & $\begin{array}{l}-1.53 \\
5 \mathrm{E}-0 \\
2\end{array}$ & \begin{tabular}{|l}
-2.29 \\
$7 \mathrm{E}-0$ \\
2
\end{tabular} & $\begin{array}{l}0.52 \\
7\end{array}$ & $\left|\begin{array}{l}0.43 \\
9\end{array}\right|$ & $\begin{array}{l}0.80 \\
7\end{array}$ & \begin{tabular}{|l|}
5.26 \\
$5 \mathrm{E}-0$ \\
2 \\
\end{tabular} & $\begin{array}{l}0.22 \\
2\end{array}$ & $\begin{array}{l}0.14 \\
5\end{array}$ & $\begin{array}{l}0.16 \\
8\end{array}$ & $\begin{array}{l}0.16 \\
4\end{array}$ & $\begin{array}{l}0.19 \\
1\end{array}$ \\
\hline V21 & $\begin{array}{l}0.81 \\
1\end{array}$ & $\begin{array}{l}0.56 \\
0\end{array}$ & $\begin{array}{l}0.43 \\
6\end{array}$ & $\begin{array}{l}2.86 \\
8 \mathrm{E}-0 \\
2\end{array}$ & \begin{tabular}{|l}
4.56 \\
$3 \mathrm{E}-0$ \\
2 \\
\end{tabular} & -.247 & $-.151 \mid$ & \begin{tabular}{|l|}
-2.84 \\
$0 \mathrm{E}-0$ \\
2 \\
\end{tabular} & \begin{tabular}{|l|}
-1.63 \\
$8 \mathrm{E}-0$ \\
2 \\
\end{tabular} & $\begin{array}{l}0.34 \\
2\end{array}$ & \begin{tabular}{|l|}
-0.11 \\
4
\end{tabular} & $\begin{array}{l}0.36 \\
0\end{array}$ & $\begin{array}{l}0.36 \\
5\end{array}$ & $\begin{array}{l}0.37 \\
2\end{array}$ & \begin{tabular}{|l|}
4.41 \\
$6 \mathrm{E}-0$ \\
2 \\
\end{tabular} & $\begin{array}{l}0.24 \\
7\end{array}$ & $\begin{array}{l}0.24 \\
7\end{array}$ & $\begin{array}{l}0.32 \\
3\end{array}$ & $\left|\begin{array}{l}0.50 \\
2\end{array}\right|$ & \begin{tabular}{|l|}
5.26 \\
$5 \mathrm{E}-0$ \\
2 \\
\end{tabular} & $\begin{array}{l}0.79 \\
4\end{array}$ & $\begin{array}{l}0.79 \\
8\end{array}$ & $\mid \begin{array}{l}0.81 \\
2\end{array}$ & $\begin{array}{l}0.80 \\
8\end{array}$ & $\begin{array}{l}0.81 \\
8\end{array}$ & $\begin{array}{l}0.80 \\
3\end{array}$ \\
\hline V22 & $\begin{array}{l}0.89 \\
4\end{array}$ & $\begin{array}{l}0.74 \\
2\end{array}$ & $\begin{array}{l}0.44 \\
1\end{array}$ & $\begin{array}{l}0.10 \\
6\end{array}$ & $\begin{array}{l}0.12 \\
7\end{array}$ & \begin{tabular}{|l|}
-0.15 \\
0
\end{tabular} & \begin{tabular}{|l|}
-0.14 \\
7
\end{tabular} & $\begin{array}{l}0.17 \\
2\end{array}$ & \begin{tabular}{|l|}
-2.61 \\
$0 \mathrm{E}-0$ \\
2 \\
\end{tabular} & $\begin{array}{l}0.33 \\
6\end{array}$ & \begin{tabular}{|l|}
-9.64 \\
$2 \mathrm{E}-0$ \\
2 \\
\end{tabular} & $\begin{array}{l}0.35 \\
4\end{array}$ & $\begin{array}{l}0.35 \\
9\end{array}$ & $\begin{array}{l}0.35 \\
3\end{array}$ & \begin{tabular}{|l|}
6.06 \\
$0 \mathrm{E}-0$ \\
2 \\
\end{tabular} & $\mid \begin{array}{l}0.24 \\
1\end{array}$ & $\begin{array}{l}0.23 \\
9\end{array}$ & $\begin{array}{l}0.33 \\
4\end{array}$ & $\left|\begin{array}{l}0.50 \\
1\end{array}\right|$ & $\begin{array}{l}0.22 \\
2\end{array}$ & $\begin{array}{l}0.79 \\
8\end{array}$ & $\begin{array}{l}0.88 \\
3\end{array}$ & $\begin{array}{l}0.84 \\
8\end{array}$ & $\begin{array}{l}0.87 \\
1\end{array}$ & $\begin{array}{l}0.87 \\
0\end{array}$ & $\begin{array}{l}0.85 \\
8\end{array}$ \\
\hline V23 & $\begin{array}{l}0.85 \\
9\end{array}$ & $\begin{array}{l}0.65 \\
4\end{array}$ & $\begin{array}{l}0.46 \\
4\end{array}$ & $\begin{array}{l}4.13 \\
0 \mathrm{E}-0 \\
2\end{array}$ & \begin{tabular}{|l|}
5.91 \\
$7 \mathrm{E}-0$ \\
2
\end{tabular} & \begin{tabular}{|l|}
-0.21 \\
1
\end{tabular} & \begin{tabular}{|l|}
-0.16 \\
1
\end{tabular} & \begin{tabular}{|l|}
4.21 \\
$8 \mathrm{E}-0$ \\
2
\end{tabular} & \begin{tabular}{|l|}
-3.53 \\
$5 \mathrm{E}-0$ \\
2 \\
\end{tabular} & $\begin{array}{l}0.36 \\
7\end{array}$ & \begin{tabular}{|l|}
-0.12 \\
1
\end{tabular} & $\begin{array}{l}0.38 \\
7\end{array}$ & $\begin{array}{l}0.39 \\
3\end{array}$ & $\begin{array}{l}0.39 \\
6\end{array}$ & \begin{tabular}{|l|}
2.56 \\
$1 \mathrm{E}-0$ \\
2 \\
\end{tabular} & $\mid \begin{array}{l}0.23 \\
9\end{array}$ & $\begin{array}{l}0.23 \\
7\end{array}$ & $\begin{array}{l}0.35 \\
4\end{array}$ & $\left|\begin{array}{l}0.53 \\
1\end{array}\right|$ & $\begin{array}{l}0.14 \\
5\end{array}$ & $\begin{array}{l}0.81 \\
2\end{array}$ & $\begin{array}{l}0.84 \\
8\end{array}$ & $\begin{array}{l}0.84 \\
4\end{array}$ & $\begin{array}{l}0.84 \\
9\end{array}$ & $\begin{array}{l}0.85 \\
4\end{array}$ & $\begin{array}{l}0.84 \\
1\end{array}$ \\
\hline V24 & $\begin{array}{l}0.88 \\
4\end{array}$ & $\begin{array}{l}0.70 \\
1\end{array}$ & $\begin{array}{l}0.43 \\
4\end{array}$ & $\begin{array}{l}8.21 \\
8 \mathrm{E}-0 \\
2 \\
\end{array}$ & .102 & $\begin{array}{l}-0.18 \\
5\end{array}$ & \begin{tabular}{|l|}
-0.15 \\
7
\end{tabular} & .116 & \begin{tabular}{|l|}
-1.33 \\
$5 \mathrm{E}-0$ \\
2 \\
\end{tabular} & $\begin{array}{l}0.32 \\
6\end{array}$ & \begin{tabular}{|l|}
-0.10 \\
8
\end{tabular} & $\begin{array}{l}0.34 \\
4\end{array}$ & $\begin{array}{l}0.35 \\
0\end{array}$ & \begin{tabular}{|l|}
0.34 \\
8
\end{tabular} & \begin{tabular}{|l|}
5.79 \\
$7 \mathrm{E}-0$ \\
2 \\
\end{tabular} & $\left|\begin{array}{l}0.26 \\
0\end{array}\right|$ & $\begin{array}{l}0.25 \\
9\end{array}$ & $\begin{array}{l}0.31 \\
9\end{array}$ & $\left|\begin{array}{l}0.49 \\
8\end{array}\right|$ & $\begin{array}{l}0.16 \\
8\end{array}$ & $\begin{array}{l}0.80 \\
8\end{array}$ & \begin{tabular}{|l|}
0.87 \\
1
\end{tabular} & $\begin{array}{l}0.84 \\
9\end{array}$ & $\begin{array}{l}0.86 \\
6\end{array}$ & $\begin{array}{l}0.86 \\
7\end{array}$ & $\begin{array}{l}0.85 \\
3\end{array}$ \\
\hline V25 & $\begin{array}{l}0.87 \\
7\end{array}$ & $\begin{array}{l}0.67 \\
3\end{array}$ & $\begin{array}{l}0.47 \\
7\end{array}$ & $\begin{array}{l}0.12 \\
4\end{array}$ & $\begin{array}{l}0.14 \\
1\end{array}$ & $\begin{array}{l}-0.16 \\
4\end{array}$ & $\begin{array}{l}-0.10 \\
7\end{array}$ & $\begin{array}{l}0.11 \\
6\end{array}$ & \begin{tabular}{|l|}
-2.95 \\
$4 \mathrm{E}-0$ \\
2 \\
\end{tabular} & $\begin{array}{l}0.38 \\
6\end{array}$ & \begin{tabular}{|l|}
-6.01 \\
$8 \mathrm{E}-0$ \\
2 \\
\end{tabular} & $\begin{array}{l}0.40 \\
5\end{array}$ & \begin{tabular}{|l}
0.41 \\
0
\end{tabular} & $\begin{array}{l}0.40 \\
0\end{array}$ & \begin{tabular}{|l|}
8.68 \\
$9 \mathrm{E}-0$ \\
2 \\
\end{tabular} & $\begin{array}{l}0.22 \\
5\end{array}$ & $\begin{array}{l}0.22 \\
4\end{array}$ & \begin{tabular}{|l|}
0.38 \\
1
\end{tabular} & $\begin{array}{l}0.53 \\
4\end{array}$ & $\begin{array}{l}0.16 \\
4\end{array}$ & $\begin{array}{l}0.81 \\
8\end{array}$ & \begin{tabular}{|l|}
0.87 \\
0
\end{tabular} & $\begin{array}{l}0.85 \\
4\end{array}$ & $\begin{array}{l}0.86 \\
7\end{array}$ & $\begin{array}{l}0.87 \\
6\end{array}$ & $\begin{array}{l}0.86 \\
4\end{array}$ \\
\hline V26 & $\begin{array}{l}0.86 \\
1\end{array}$ & $\begin{array}{l}0.66 \\
8\end{array}$ & $\begin{array}{l}0.50 \\
0\end{array}$ & $\begin{array}{l}0.15 \\
3\end{array}$ & $\begin{array}{l}0.16 \\
8\end{array}$ & \begin{tabular}{|l|}
-0.12 \\
8
\end{tabular} & \begin{tabular}{|l|}
-7.11 \\
$2 \mathrm{E}-0$ \\
2
\end{tabular} & $\begin{array}{l}0.13 \\
9\end{array}$ & \begin{tabular}{|l|}
-3.28 \\
$6 \mathrm{E}-0$ \\
2 \\
\end{tabular} & $\begin{array}{l}0.42 \\
0\end{array}$ & \begin{tabular}{|l|}
-2.58 \\
$2 \mathrm{E}-0$ \\
2
\end{tabular} & $\begin{array}{l}0.44 \\
0\end{array}$ & $\begin{array}{l}0.44 \\
5\end{array}$ & \begin{tabular}{|l|}
0.42 \\
9
\end{tabular} & \begin{tabular}{|l|}
9.46 \\
$0 \mathrm{E}-0$ \\
2
\end{tabular} & $\begin{array}{l}0.20 \\
6\end{array}$ & $\begin{array}{l}0.20 \\
5\end{array}$ & \begin{tabular}{|l}
0.41 \\
8
\end{tabular} & $\mid \begin{array}{l}0.55 \\
2\end{array}$ & $\begin{array}{l}0.19 \\
1\end{array}$ & $\begin{array}{l}0.80 \\
3\end{array}$ & $\begin{array}{l}0.85 \\
8\end{array}$ & $\begin{array}{l}0.84 \\
1\end{array}$ & $\begin{array}{l}0.85 \\
3\end{array}$ & $\begin{array}{l}0.86 \\
4\end{array}$ & $\begin{array}{l}0.85 \\
6\end{array}$ \\
\hline
\end{tabular}


(Table 9 continued)

\begin{tabular}{|c|c|c|c|c|c|c|c|c|c|c|c|c|c|c|c|c|c|c|c|c|c|c|c|c|c|c|c|}
\hline \multirow{2}{*}{\multicolumn{2}{|c|}{\begin{tabular}{|l|l} 
Residu & V \\
al
\end{tabular}}} & V1 & V2 & V3 & V4 & V5 & V6 & V7 & V8 & V9 & V10 & V11 & V12 & V13 & V14 & V15 & $\mathrm{V} 16$ & $\begin{array}{ll}\text { V17 } \\
\end{array}$ & V18 & V19 & V20 & V21 & V22 & V23 & V24 & \multirow{2}{*}{\begin{tabular}{|l}
$\mathrm{V} 25$ \\
-4.08 \\
$1 \mathrm{E}-0$ \\
2 \\
\end{tabular}} & \multirow{2}{*}{\begin{tabular}{|l}
26 \\
-3.81 \\
$1 \mathrm{E}-0$ \\
2 \\
\end{tabular}} \\
\hline \multirow[t]{37}{*}{$\begin{array}{l}\text { Residu } \\
\text { al }\end{array}$} & & & $\begin{array}{l}-2.23 \\
5 \mathrm{E}-0 \\
2\end{array}$ & \begin{tabular}{|l}
2.65 \\
$0 \mathrm{E}-0$ \\
4
\end{tabular} & $\begin{array}{l}-1.75 \\
4 \mathrm{E}-0 \\
2\end{array}$ & $\begin{array}{l}-2.23 \\
9 \mathrm{E}-0 \\
2\end{array}$ & $\begin{array}{l}-6.58 \\
4 \mathrm{E}-0 \\
3\end{array}$ & $\begin{array}{l}2.75 \\
1 \mathrm{E}-0 \\
2\end{array}$ & $\begin{array}{l}2.36 \\
1 \mathrm{E}-0 \\
2\end{array}$ & $\begin{array}{l}-2.30 \\
2 \mathrm{E}-0 \\
2\end{array}$ & $\begin{array}{l}1.27 \\
6 \mathrm{E}-0 \\
2\end{array}$ & \begin{tabular}{|l}
2.63 \\
$6 \mathrm{E}-0$ \\
2
\end{tabular} & $\begin{array}{l}7.52 \\
0 \mathrm{E}-0 \\
3\end{array}$ & $\begin{array}{l}4.15 \\
4 \mathrm{E}-0 \\
3\end{array}$ & \begin{tabular}{|l|}
5.01 \\
$0 \mathrm{E}-0$ \\
3
\end{tabular} & $\begin{array}{l}-1.68 \\
8 \mathrm{E}-0 \\
2\end{array}$ & $\begin{array}{l}8.51 \\
3 \mathrm{E}-0 \\
3\end{array}$ & $\begin{array}{l}8.96 \\
6 \mathrm{E}-0 \\
3\end{array}$ & $\begin{array}{l}-2.54 \\
1 \mathrm{E}-0 \\
3\end{array}$ & \begin{tabular}{|l}
-1.31 \\
$9 \mathrm{E}-0$ \\
2
\end{tabular} & $\begin{array}{l}-2.92 \\
5 \mathrm{E}-0 \\
2\end{array}$ & $\begin{array}{l}-5.61 \\
1 \mathrm{E}-0 \\
2\end{array}$ & $\begin{array}{l}4.38 \\
1 \mathrm{E}-0 \\
2\end{array}$ & $\begin{array}{l}2.36 \\
2 \mathrm{E}-0 \\
3\end{array}$ & $\begin{array}{l}1.80 \\
7 \mathrm{E}-0 \\
2\end{array}$ & & \\
\hline & & -2.23 & & -6.11 & $\mid-2.06$ & \begin{tabular}{|l|l|}
-7.43 \\
\end{tabular} & 4.67 & 4.45 & $\mid-7.56$ & -1.12 & 1.03 & 3.75 & 4.61 & -2.65 & $\mid-9.61$ & 2.99 & -1.64 & $\mid-5.54$ & \begin{tabular}{|l}
5.23 \\
\end{tabular} & -2.73 & -2.71 & -1.25 & -9.96 & \begin{tabular}{|l}
8.70 \\
\end{tabular} & -1.54 & & 7.79 \\
\hline & V2 & $\begin{array}{l}5 \mathrm{E}-0 \\
2\end{array}$ & & $\begin{array}{l}2 \mathrm{E}-0 \\
3\end{array}$ & $\begin{array}{l}1 \mathrm{E}-0 \\
2\end{array}$ & $\begin{array}{l}7 \mathrm{E}-0 \\
3\end{array}$ & $\begin{array}{l}0 \mathrm{E}-0 \\
3\end{array}$ & $\begin{array}{l}6 \mathrm{E}-0 \\
2\end{array}$ & $\begin{array}{l}8 \mathrm{E}-0 \\
2\end{array}$ & $\begin{array}{l}9 \mathrm{E}-0 \\
2\end{array}$ & $\begin{array}{l}3 \mathrm{E}-0 \\
2\end{array}$ & $\begin{array}{l}2 \mathrm{E}-0 \\
2\end{array}$ & $\begin{array}{l}5 \mathrm{E}-0 \\
3\end{array}$ & $\begin{array}{l}2 \mathrm{E}-0 \\
3\end{array}$ & $\begin{array}{l}3 \mathrm{E}-0 \\
3\end{array}$ & $\begin{array}{l}3 \mathrm{E}-0 \\
2\end{array}$ & $\begin{array}{l}8 \mathrm{E}-0 \\
3\end{array}$ & $\begin{array}{l}5 \mathrm{E}-0 \\
4\end{array}$ & $\begin{array}{l}0 \mathrm{E}-0 \\
3\end{array}$ & $\begin{array}{l}3 \mathrm{E}-0 \\
2\end{array}$ & $\begin{array}{l}2 \mathrm{E}-0 \\
2\end{array}$ & $\begin{array}{l}0 \mathrm{E}-0 \\
2\end{array}$ & $\begin{array}{l}3 \mathrm{E}-0 \\
3\end{array}$ & $\begin{array}{l}3 \mathrm{E}-0 \\
3\end{array}$ & $\begin{array}{l}0 \mathrm{E}-0 \\
2\end{array}$ & $\begin{array}{l}4 \mathrm{E}-0 \\
2\end{array}$ & $\begin{array}{l}0 \mathrm{E}-0 \\
3\end{array}$ \\
\hline & V3 & \begin{tabular}{|l}
2.65 \\
$0 \mathrm{E}-0$ \\
4
\end{tabular} & $\begin{array}{l}-6.11 \\
2 \mathrm{E}-0 \\
3\end{array}$ & & $\begin{array}{l}5.81 \\
8 \mathrm{E}-0 \\
2\end{array}$ & $\begin{array}{l}7.12 \\
6 \mathrm{E}-0 \\
2\end{array}$ & $\begin{array}{l}-7.38 \\
7 \mathrm{E}-0 \\
2\end{array}$ & \begin{tabular}{|l}
-8.62 \\
$6 \mathrm{E}-0$ \\
2
\end{tabular} & $\begin{array}{l}5.95 \\
3 \mathrm{E}-0 \\
2\end{array}$ & $\begin{array}{l}-3.74 \\
9 \mathrm{E}-0 \\
2\end{array}$ & $\begin{array}{l}-4.34 \\
2 \mathrm{E}-0 \\
2\end{array}$ & $\begin{array}{l}-9.48 \\
2 \mathrm{E}-0 \\
2\end{array}$ & $\begin{array}{l}-3.61 \\
7 \mathrm{E}-0 \\
2\end{array}$ & $\begin{array}{l}-2.79 \\
7 \mathrm{E}-0 \\
2\end{array}$ & $\begin{array}{l}-0.10 \\
6\end{array}$ & $\begin{array}{l}4.48 \\
2 \mathrm{E}-0 \\
2\end{array}$ & $\begin{array}{l}3.72 \\
4 \mathrm{E}-0 \\
2\end{array}$ & $\begin{array}{l}3.62 \\
2 \mathrm{E}-0 \\
2\end{array}$ & $\begin{array}{l}-1.45 \\
6 \mathrm{E}-0 \\
2\end{array}$ & 0.13 & $\begin{array}{l}-3.21 \\
1 \mathrm{E}-0 \\
2\end{array}$ & $\begin{array}{l}-7.17 \\
3 \mathrm{E}-0 \\
2\end{array}$ & $\begin{array}{l}-1.62 \\
6 \mathrm{E}-0 \\
2\end{array}$ & $\begin{array}{l}1.64 \\
5 \mathrm{E}-0 \\
2\end{array}$ & $\begin{array}{l}-9.25 \\
9 \mathrm{E}-0 \\
2\end{array}$ & \begin{tabular}{|l|}
-4.38 \\
$4 \mathrm{E}-0$ \\
2
\end{tabular} & $\begin{array}{l}-3.49 \\
3 \mathrm{E}-0 \\
2\end{array}$ \\
\hline & & -1.75 & -2.06 & 5.81 & & 7.26 & -8.29 & -6.65 & 1.49 & 9.21 & -1.20 & -5.18 & -8.83 & -3.50 & -4.42 & -8.69 & 6.09 & 3.43 & 2.46 & 5.60 & -2.95 & -1.66 & 3.45 & 2.12 & -3.54 & -1.75 & -1.92 \\
\hline & V4 & $4 \mathrm{E}-0$ & $1 \mathrm{E}-0$ & $8 \mathrm{E}-0$ & & $7 \mathrm{E}-0$ & $4 \mathrm{E}-0$ & $3 \mathrm{E}-0$ & $3 \mathrm{E}-0$ & $8 \mathrm{E}-0$ & $3 \mathrm{E}-0$ & $5 \mathrm{E}-0$ & $9 \mathrm{E}-0$ & 9E-0 & $0 \mathrm{E}-0$ & $8 \mathrm{E}-0$ & $0 \mathrm{E}-0$ & $6 \mathrm{E}-0$ & $4 \mathrm{E}-0$ & $4 \mathrm{E}-0$ & $3 \mathrm{E}-0$ & $3 \mathrm{E}-0$ & $2 \mathrm{E}-0$ & $6 \mathrm{E}-0$ & 9E-0 & $6 \mathrm{E}-0$ & E-0 \\
\hline & & 2 & 2 & 2 & & 2 & 2 & 2 & 2 & 3 & 2 & 2 & 3 & 3 & 2 & 2 & 3 & 3 & 2 & $a_{0}$ & 3 & 2 & 3 & 2 & 2 & 2 & 2 \\
\hline & & -2.23 & -7.43 & 7.12 & 7.26 & & -8.71 & -8.60 & 3.25 & 2.09 & -2.14 & -7.61 & $\mid-1.93$ & -1.46 & -2.32 & -6.63 & 2.50 & -1.04 & \begin{tabular}{|l|}
1.74 \\
\end{tabular} & 9.57 & -2.22 & $\mid-2.46$ & -8.39 & 7.04 & -4.14 & -2.15 & -1.50 \\
\hline & V5 & 9E-0 & 7E-0 & $6 \mathrm{E}-0$ & $7 \mathrm{E}-0$ & & 9E-0 & $5 \mathrm{E}-0$ & $8 \mathrm{E}-0$ & $3 \mathrm{E}-0$ & $6 \mathrm{E}-0$ & 9E-0 & $2 \mathrm{E}-0$ & $0 \mathrm{E}-0$ & $0 \mathrm{E}-0$ & 9E-0 & $2 \mathrm{E}-0$ & $5 \mathrm{E}-0$ & $2 \mathrm{E}-0$ & $2 \mathrm{E}-0$ & $8 \mathrm{E}-0$ & 9E-0 & $5 \mathrm{E}-0$ & 9E-0 & $5 \mathrm{E}-0$ & $6 \mathrm{E}-0$ & $4 \mathrm{E}-0$ \\
\hline & & 2 & 3 & 2 & 2 & & 2 & 2 & 3 & 2 & 2 & 2 & 2 & 2 & 2 & 2 & 3 & 4 & 2 & 2 & 3 & 2 & 3 & 3 & 2 & 2 & 2 \\
\hline & & -6.58 & 4.67 & -7.38 & $\mid-8.29$ & -8.71 & & 8.91 & -6.80 & -1.20 & -8.77 & 2.96 & -1.03 & -1.22 & 1.15 & -3.04 & -3.04 & -2.99 & -2.52 & -6.91 & -5.67 & \begin{tabular}{|l|}
9.39 \\
\end{tabular} & -4.08 & 3.60 & 3.90 & 4.46 & 3.46 \\
\hline & V6 & $4 \mathrm{E}-0$ & $0 \mathrm{E}-0$ & 7E-0 & $4 \mathrm{E}-0$ & $9 \mathrm{E}-0$ & & $0 \mathrm{E}-0$ & $8 \mathrm{E}-0$ & $4 \mathrm{E}-0$ & $0 \mathrm{E}-0$ & 7E-0 & $5 \mathrm{E}-0$ & $9 \mathrm{E}-0$ & $3 \mathrm{E}-0$ & $0 \mathrm{E}-0$ & $9 \mathrm{E}-0$ & 9E-0 & $6 \mathrm{E}-0$ & $3 \mathrm{E}-0$ & $8 \mathrm{E}-0$ & $1 \mathrm{E}-0$ & $1 \mathrm{E}-0$ & $1 \mathrm{E}-0$ & 7E-0 & $3 \mathrm{E}-0$ & $9 \mathrm{E}-0$ \\
\hline & & 3 & 3 & 2 & 2 & 2 & & 2 & 2 & 2 & 3 & 2 & 2 & 2 & 2 & 2 & 2 & 2 & 2 & 2 & 2 & 2 & 3 & 2 & 2 & 2 & 2 \\
\hline & & 2.75 & 4.45 & -8.62 & -6.65 & -8.60 & 8.91 & & -6.31 & -5.67 & 2.64 & & $\mid-5.10$ & -9.78 & 2.75 & & -1.71 & -1.76 & -3.06 & & -2.44 & 4.96 & 9.61 & 1.54 & 5.52 & 3.10 & 3.24 \\
\hline & V7 & $1 \mathrm{E}-0$ & $6 \mathrm{E}-0$ & $6 \mathrm{E}-0$ & $3 E-0$ & $5 \mathrm{E}-0$ & $0 \mathrm{E}-0$ & & $8 \mathrm{E}-0$ & $5 \mathrm{E}-0$ & 7E-0 & & $1 \mathrm{E}-0$ & $2 \mathrm{E}-0$ & $5 \mathrm{E}-0$ & & $3 \mathrm{E}-0$ & $8 \mathrm{E}-0$ & $2 \mathrm{E}-0$ & & $7 \mathrm{E}-0$ & $2 \mathrm{E}-0$ & $2 \mathrm{E}-0$ & $2 \mathrm{E}-0$ & $5 \mathrm{E}-0$ & $9 \mathrm{E}-0$ & 7E-0 \\
\hline & & 2 & 2 & 2 & 2 & 2 & 2 & & 2 & 2 & 3 & & 3 & 3 & 3 & & 2 & 2 & 2 & & 2 & ת & 3 & 2 & 2 & 2 & 2 \\
\hline & & 2.36 & -7.56 & 5.95 & 1.49 & 3.25 & -6.80 & -6.31 & & -3.12 & 1.48 & -4.80 & 1.31 & 1.11 & 3.33 & -5.13 & 3.32 & 3.21 & 8.88 & 5.40 & -9.85 & -3.86 & -1.45 & -2.20 & -1.50 & -9.54 & -4.64 \\
\hline & V8 & $1 \mathrm{E}-0$ & $8 \mathrm{E}-0$ & $3 \mathrm{E}-0$ & $3 \mathrm{E}-0$ & $8 \mathrm{E}-0$ & $8 \mathrm{E}-0$ & $8 \mathrm{E}-0$ & & $7 \mathrm{E}-0$ & $0 \mathrm{E}-0$ & $8 \mathrm{E}-0$ & $4 \mathrm{E}-0$ & $1 \mathrm{E}-0$ & $1 \mathrm{E}-0$ & $6 \mathrm{E}-0$ & $3 \mathrm{E}-0$ & $8 \mathrm{E}-0$ & $2 \mathrm{E}-0$ & $9 \mathrm{E}-0$ & $3 \mathrm{E}-0$ & $0 \mathrm{E}-0$ & $5 \mathrm{E}-0$ & $2 \mathrm{E}-0$ & $4 \mathrm{E}-0$ & $8 \mathrm{E}-0$ & $7 \mathrm{E}-0$ \\
\hline & & 2 & 2 & $a_{0}$ & 2 & 3 & 2 & 2 & & 2 & 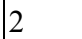 & 2 & 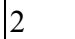 & 2 & 2 & 2 & $\sigma_{0}$ & 2 & 3 & & 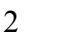 & 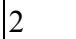 & & 2 & 2 & ? & 3 \\
\hline & & -2.30 & -1.12 & 3.74 & 9.21 & 2.09 & -1.20 & -5.67 & -3.12 & & -2.43 & -4.84 & 2.17 & 2.58 & 6.12 & \begin{tabular}{|l|l|}
4.39 \\
\end{tabular} & -7.18 & -6.88 & 1.99 & 3.46 & \begin{tabular}{|l|l|}
5.60 \\
\end{tabular} & 2.63 & -1.34 & -1.72 & $\begin{array}{ll}1.87 \\
\end{array}$ & 1.49 & 1.25 \\
\hline & V9 & $2 \mathrm{E}-0$ & 9E-0 & $9 \mathrm{E}-0$ & $8 \mathrm{E}-0$ & $3 \mathrm{E}-0$ & $4 \mathrm{E}-0$ & $5 \mathrm{E}-0$ & $7 \mathrm{E}-0$ & & $7 \mathrm{E}-0$ & 0E-0 & $2 \mathrm{E}-0$ & 7E-0 & $1 \mathrm{E}-0$ & 9E-0 & $3 \mathrm{E}-0$ & $6 \mathrm{E}-0$ & $6 \mathrm{E}-0$ & $5 \mathrm{E}-0$ & $7 \mathrm{E}-0$ & $6 \mathrm{E}-0$ & $6 \mathrm{E}-0$ & $1 \mathrm{E}-0$ & $6 \mathrm{E}-0$ & $1 \mathrm{E}-0$ & $5 \mathrm{E}-0$ \\
\hline & & 2 & 2 & 2 & 3 & 2 & 2 & 2 & 2 & & 3 & 2 & 3 & 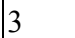 & 3 & 2 & 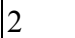 & 2 & o & $a_{0}$ & 2 & 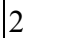 & 2 & 2 & 2 & 2 & 2 \\
\hline & & \begin{tabular}{|l|}
1.27 \\
\end{tabular} & 1.03 & -4.34 & -1.20 & -2.14 & \begin{tabular}{|l}
-8.77 \\
\end{tabular} & 2.64 & 1.48 & -2.43 & & -8.13 & 4.32 & -9.26 & -4.43 & -1.24 & 1.08 & \begin{tabular}{|l|l}
1.11 \\
\end{tabular} & 1.89 & -5.69 & -2.09 & -2.37 & 1.28 & -2.25 & 1.26 & 1.28 & 9.16 \\
\hline & V10 & $6 \mathrm{E}-0$ & $3 \mathrm{E}-0$ & $2 \mathrm{E}-0$ & $3 \mathrm{E}-0$ & $6 \mathrm{E}-0$ & OE-0 & $7 \mathrm{E}-0$ & $0 \mathrm{E}-0$ & $7 \mathrm{E}-0$ & & $9 \mathrm{E}-0$ & $2 \mathrm{E}-0$ & $8 \mathrm{E}-0$ & $9 \mathrm{E}-0$ & $8 \mathrm{E}-0$ & $1 \mathrm{E}-0$ & $5 \mathrm{E}-0$ & $8 \mathrm{E}-0$ & $0 \mathrm{E}-0$ & $8 \mathrm{E}-0$ & $6 \mathrm{E}-0$ & $4 \mathrm{E}-0$ & 0E-0 & $6 \mathrm{E}-0$ & $2 \mathrm{E}-0$ & 7E-0 \\
\hline & & 2 & 2 & 2 & 2 & 2 & $\sigma_{0}$ & 3 & 2 & 3 & & 4 & 2 & 3 & 2 & 3 & 2 & 2 & 4 & $I^{2}$ & 2 & 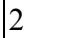 & 2 & 2 & 2 & 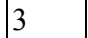 & 3 \\
\hline & & 2.63 & 3.75 & -9.48 & -5.18 & $\mid-7.61$ & 2.96 & & -4.80 & -4.84 & -8.13 & & -2.60 & 3.98 & 2.19 & & -1.20 & -1.27 & -2.44 & & -1.77 & 4.12 & 5.71 & 9.70 & 5.06 & 2.42 & 2.25 \\
\hline & V11 & $6 \mathrm{E}-0$ & $2 \mathrm{E}-0$ & $2 \mathrm{E}-0$ & $5 \mathrm{E}-0$ & $9 \mathrm{E}-0$ & $7 \mathrm{E}-0$ & & $8 \mathrm{E}-0$ & $0 \mathrm{E}-0$ & $9 \mathrm{E}-0$ & & $3 \mathrm{E}-0$ & $5 \mathrm{E}-0$ & $0 \mathrm{E}-0$ & & $1 \mathrm{E}-0$ & $1 \mathrm{E}-0$ & $4 \mathrm{E}-0$ & & $2 \mathrm{E}-0$ & $2 \mathrm{E}-0$ & $4 \mathrm{E}-0$ & $4 \mathrm{E}-0$ & $3 \mathrm{E}-0$ & $0 \mathrm{E}-0$ & $0 \mathrm{E}-0$ \\
\hline & & 2 & 2 & 5 & 2 & 2 & 5 & & 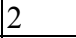 & 5 & 4 & & 4 & 3 & 2 & & 2 & 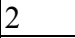 & 2 & & 3 & 2 & 3 & 3 & 2 & 2 & 2 \\
\hline & & 7.52 & 4.61 & $\mid-3.61$ & -8.83 & -1.93 & -1.03 & -5.10 & 1.31 & 2.17 & 4.32 & -2.60 & & 8.78 & $\mid-1.68$ & 1.23 & 6.96 & 7.44 & \begin{tabular}{|l|}
1.01 \\
\end{tabular} & -3.75 & -9.58 & -1.50 & 7.54 & -2.21 & 1.10 & 2.02 & 7.32 \\
\hline & V12 & $0 \mathrm{E}-0$ & $5 \mathrm{E}-0$ & $7 \mathrm{E}-0$ & $9 \mathrm{E}-0$ & $2 \mathrm{E}-0$ & $5 \mathrm{E}-0$ & $1 \mathrm{E}-0$ & $4 \mathrm{E}-0$ & $2 \mathrm{E}-0$ & $2 \mathrm{E}-0$ & $3 \mathrm{E}-0$ & & $1 \mathrm{E}-0$ & $1 \mathrm{E}-0$ & $3 \mathrm{E}-0$ & $6 \mathrm{E}-0$ & $1 \mathrm{E}-0$ & $0 \mathrm{E}-0$ & $1 \mathrm{E}-0$ & $8 \mathrm{E}-0$ & $4 \mathrm{E}-0$ & $6 \mathrm{E}-0$ & $0 \mathrm{E}-0$ & $6 \mathrm{E}-0$ & OE-0 & $2 \mathrm{E}-0$ \\
\hline & & 3 & 3 & 2 & 3 & 2 & 2 & 3 & 2 & 促 & 2 & 4 & & 3 & 2 & 2 & 2 & 3 & 2 & 2 & 3 & 2 & 3 & 2 & 2 & 3 & 3 \\
\hline & & 4.15 & -2.65 & 2.79 & -3.50 & -1.46 & -1.22 & -9.78 & 1.11 & 2.58 & -9.26 & 3.98 & 8.78 & & 7.50 & 1.56 & 3.95 & 4.11 & 1.74 & -1.43 & 4.08 & -6.93 & 3.37 & -2.01 & 9.88 & 2.04 & 4.79 \\
\hline & V13 & $4 \mathrm{E}-0$ & $2 \mathrm{E}-0$ & 7E-0 & 9E-0 & $0 \mathrm{E}-0$ & $9 \mathrm{E}-0$ & $2 \mathrm{E}-0$ & $1 \mathrm{E}-0$ & $7 \mathrm{E}-0$ & $8 \mathrm{E}-0$ & $5 \mathrm{E}-0$ & $1 \mathrm{E}-0$ & & $6 \mathrm{E}-1$ & $1 \mathrm{E}-\mathrm{C}$ & $3 \mathrm{E}-0$ & $4 \mathrm{E}-0$ & $3 E-$ & $3 \mathrm{E}-0$ & $4 \mathrm{E}-0$ & $8 \mathrm{E}-0$ & $3 \mathrm{E}-0$ & 9E-0 & $6 \mathrm{E}-0$ & $2 \mathrm{E}-0$ & $2 \mathrm{E}$ \\
\hline & & 3 & 3 & 2 & 3 & 2 & 2 & 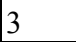 & 2 & 3 & 3 & 3 & 3 & & 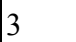 & 2 & 3 & 3 & 2 & 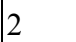 & 4 & 3 & 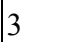 & 2 & 3 & 3 & 3 \\
\hline & & 5.01 & -9.61 & & -4.42 & -2.32 & 1.15 & 2.75 & 3.33 & 6.12 & $\mid-4.43$ & 2.19 & -1.68 & 7.50 & & 7.32 & -3.27 & -2.39 & -1.14 & -3.67 & 2.85 & 7.04 & 1.35 & 1.17 & 3.23 & -9.85 & -2.0 \\
\hline & V14 & -0 & $3 \mathrm{E}-0$ & & $0 \mathrm{E}-0$ & $0 \mathrm{E}-0$ & $3 \mathrm{E}-0$ & $5 \mathrm{E}-0$ & $1 \mathrm{E}-0$ & $1 \mathrm{E}-0$ & $9 \mathrm{E}-0$ & $0 \mathrm{E}-0$ & $1 \mathrm{E}-0$ & $6 \mathrm{E}$ & & $6 \mathrm{E}-0$ & $6 \mathrm{E}-0$ & $0 \mathrm{E}-0$ & $1 \mathrm{E}-0$ & $5 \mathrm{E}-0$ & $6 \mathrm{E}-0$ & $5 \mathrm{E}-0$ & $6 \mathrm{E}-0$ & $4 \mathrm{E}-0$ & $2 \mathrm{E}-0$ & $3 \mathrm{E}-0$ & $2 \mathrm{E}$ \\
\hline & & 3 & 3 & & 2 & , & & 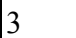 & 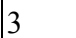 & & 2 & 0 & a & 3 & & o & 3 & & 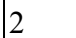 & & & 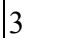 & & & o & & 2 \\
\hline
\end{tabular}


(Table 9 continued)

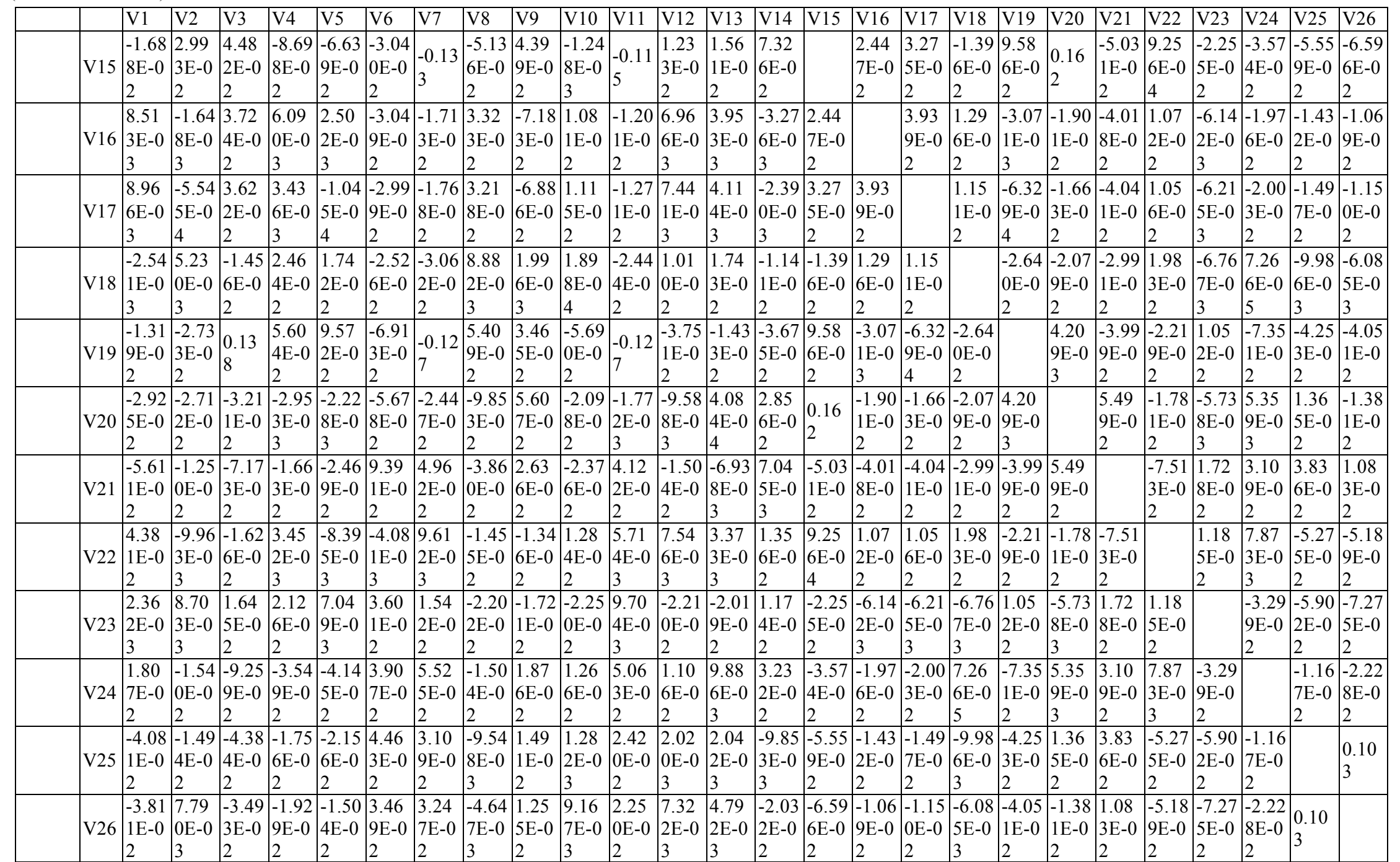

Notes. Extraction Method: Principal Component Analysis; residuals are computed between observed and reproduced correlations.

There are $58(17.0 \%)$ nonredundant residuals with absolute values $>0.05$; reproduced communalities. 
Table 10

Rotated Component Matrix ${ }^{a}$

\begin{tabular}{|c|c|c|c|c|c|}
\hline & Component & & & & \\
\hline & 1 & 2 & 3 & 4 & 5 \\
\hline \begin{tabular}{|l|} 
V1 \\
\end{tabular} & 0.936 & 0.126 & $-3.799 \mathrm{E}-02$ & 8.493E-02 & 0.106 \\
\hline V2 & 0.714 & 0.126 & $-5.887 \mathrm{E}-02$ & 8.752E-02 & 0.578 \\
\hline V3 & 0.339 & 0.726 & 9.249E-02 & $-7.458 \mathrm{E}-02$ & 7.790E-02 \\
\hline \begin{tabular}{|l|} 
V4 \\
\end{tabular} & $5.009 \mathrm{E}-02$ & 0.197 & 0.902 & 0.105 & 0.113 \\
\hline V5 & 7.798E-02 & 0.164 & 0.886 & 0.111 & 0.114 \\
\hline \begin{tabular}{|l} 
V6 \\
\end{tabular} & -0.286 & 0.304 & 0.573 & 0.148 & 0.432 \\
\hline V7 & -0.255 & 0.388 & 0.738 & 0.179 & $3.278 \mathrm{E}-02$ \\
\hline V8 & 0.102 & 1.694E-02 & 0.653 & $6.237 \mathrm{E}-02$ & 0.601 \\
\hline \begin{tabular}{|l|} 
V9 \\
\end{tabular} & $-6.038 \mathrm{E}-02$ & -0.102 & 0.344 & 0.831 & $2.933 \mathrm{E}-02$ \\
\hline \begin{tabular}{|l} 
V10 \\
\end{tabular} & 0.194 & 0.886 & 0.200 & -0.120 & $8.295 \mathrm{E}-02$ \\
\hline \begin{tabular}{|l|} 
V11 \\
\end{tabular} & -0.194 & 0.344 & 0.792 & 0.193 & $4.134 \mathrm{E}-02$ \\
\hline \begin{tabular}{|l|} 
V12 \\
\end{tabular} & 0.202 & 0.932 & 0.190 & $-8.829 \mathrm{E}-02$ & 0.102 \\
\hline \begin{tabular}{|l} 
V13 \\
\end{tabular} & 0.206 & 0.939 & 0.174 & $-9.003 \mathrm{E}-02$ & 0.109 \\
\hline \begin{tabular}{|l|} 
V14 \\
\end{tabular} & 0.216 & 0.873 & 4.492E-02 & $-8.402 \mathrm{E}-02$ & 7.406E-02 \\
\hline \begin{tabular}{|l|} 
V15 \\
\end{tabular} & 0.103 & -0.112 & 0.649 & $-9.910 \mathrm{E}-03$ & \begin{tabular}{|l|}
-0.189 \\
\end{tabular} \\
\hline \begin{tabular}{|l|} 
V16 \\
\end{tabular} & 0.237 & -0.149 & 8.708E-02 & 0.931 & $3.289 \mathrm{E}-02$ \\
\hline \begin{tabular}{|l|} 
V17 \\
\end{tabular} & 0.237 & -0.153 & 8.995E-02 & 0.931 & $2.521 \mathrm{E}-02$ \\
\hline \begin{tabular}{|l} 
V18 \\
\end{tabular} & 0.188 & 0.890 & 0.262 & -0.161 & 0.124 \\
\hline \begin{tabular}{|l|} 
V19 \\
\end{tabular} & 0.390 & 0.749 & $1.842 \mathrm{E}-02$ & 7.894E-02 & $9.630 \mathrm{E}-02$ \\
\hline V20 & $6.525 \mathrm{E}-02$ & 0.451 & $6.906 \mathrm{E}-02$ & $5.192 \mathrm{E}-03$ & 0.771 \\
\hline \begin{tabular}{|l|}
$\mathrm{V} 21$ \\
\end{tabular} & 0.838 & 0.243 & $-6.213 \mathrm{E}-02$ & 0.102 & -0.140 \\
\hline \begin{tabular}{|l} 
V22 \\
\end{tabular} & 0.916 & 0.172 & $1.029 \mathrm{E}-02$ & $4.825 \mathrm{E}-02$ & 0.108 \\
\hline V23 & 0.878 & 0.249 & $-6.326 \mathrm{E}-02$ & 7.896E-02 & $-2.596 \mathrm{E}-02$ \\
\hline V24 & 0.909 & 0.178 & $-1.186 \mathrm{E}-02$ & 7.551E-02 & $3.781 \mathrm{E}-02$ \\
\hline \begin{tabular}{|l|}
$\mathrm{V} 25$ \\
\end{tabular} & 0.903 & 0.238 & $3.070 \mathrm{E}-02$ & $4.686 \mathrm{E}-02$ & $-6.145 \mathrm{E}-03$ \\
\hline V26 & 0.882 & 0.272 & 5.609E-02 & $3.447 \mathrm{E}-02$ & $8.707 \mathrm{E}-03$ \\
\hline
\end{tabular}

Notes. Extraction Method: Principal Component Analysis. Rotation Method: Varimax with Kaiser Normalization. 


\section{Table 11}

Component Transformation Matrix

\begin{tabular}{llllll}
\hline Component & 1 & 2 & 3 & 4 & 5 \\
\hline 1 & 0.666 & 0.676 & 0.250 & 0.052 & 0.023 \\
2 & -0.636 & 0.330 & 0.679 & 0.712 & 0.159 \\
3 & 0.255 & -0.484 & 0.435 & 0.689 & 0.063 \\
4 & -0.295 & 0.405 & -0.517 & -0.120 & 0.084 \\
5 & -0.014 & -0.188 & -0.143 & 0.964 \\
\hline
\end{tabular}

Notes. Extraction Method: Principal Component Analysis. Rotation Method: Varimax with Kaiser Normalization. 


\section{Conclusions}

The main conclusions of this study may be summarized and 385 consumers were surveyed to find out how they evaluate banks operating in the State of Libya. The main results of the survey are:

1. The respondents were asked to rate the importance of 26 bank attributes. Factor Analysis was used to reduce the number of explanatory variables to a manageable level. Bartlett's test of sphericity and Kaiser-Meyer-Olkin (KMO) measure of sampling suggest that Factor Analysis is appropriate in determining the main reasons for preferring one type of bank over the other within the dual-banking system currently operating in Libya.

2. Using the principal component method and varimax rotation, the 26 explanatory variables for dealing with a particular bank were reduced to the five factors, namely, a convenience factor, a traditional services factor, a reputation factor, a cost-return factor, and a religious factor.

3. Libyan consumers who elect to bank with conventional local banks do so mainly for convenience and because of the reputation these banks enjoy locally.

4. Libyan consumers who elect to bank with Islamic banks do so mainly for religious reasons. Adhering to Islamic religion, which prohibits using (i.e., paying or receiving) interest is a main factor which motivates Libyan consumers to deal (borrow or deposit funds) with local Islamic bank rather than any of the other traditional (interest-based, local, or foreign) banks.

5. While reputation and high-quality banking services are important factors when selecting a bank, commercial banks must pay attention to price-competition when thinking of expanding or maintaining their market shares. Banking deregulation should be a base for competition in terms of interest rates on loans and deposits.

6. Although Libya is a relatively small country, convenience, in terms of number of branches, location of branches, and business hours, is an important decision factor for consumers who live in relatively new developed areas.

\section{References}

Bartholomew, D. J., \& Knott, M. (1999). Latent variable models and factor analysis. London, Edward Arnold Publishers.

Basilevsky, A. (1994). Statistical factor analysis and related methods. New York, N.Y.: John Wiley.

Coakes, S. J., \& Steed, L. G. (1999). SPSS: Analysis without anguish. Sydney: Jacaranda Wiley Ltd.

Dillon, W. R., \& Goldstein, M. (1984). Multivariate analysis: Methods and applications. New York, N.Y.: John Wiley.

Ding, A. A. (1999). Prediction intervals, factor analysis models and high-dimensional empirical linear predictions. Journal of the American Statistical Association, 94, 442-451.

Dunteman, G. H. (1989). Principal component analysis. Newburg Park, CA: Sage Publications.

Goldstein, M. (1984). Multivariate analysis: Methods and applications. New York, N.Y.: John Wiley.

Hair, J., Anderson, R. E., Joseph, R. L., Tatham, F., \& Blach, W. C. (2004). Multivariate data analysis with readings (10th ed.). New York, N.Y.: Macmulton.

Harman, H. H. (1967). Modern factor analysis. Chicago: University of Chicago Press.

Johnson, R. A., \& Wichern, D. W. (2002). Applied multivariate statistical analysis. Englewood Cliffs, N.J.: Prentice-Hall.

Joreskog, R. G., \& Sorborn, D. (1979). Advances in factor analysis and structural equation models. Cambridge: Mass Abt Books. Lattin, J., Carroll, J. D., \& Green, P. E. (2003). Analyzing multivariate data. Mason, USA: Thomson.

Lawley, D. N., \& Maxwell, A. K. (1971). Factor analysis as a statistical method. New York, N.Y.: American Elsevier Publishing Company.

Malhotra, N. K. (2004). Marketing research: An applied orientation. London: Prentice-Hall.

Manly, B. F. J. (1994). Multivariate statistical methods (2nd ed.). London: Champon \& Hall. 
Metwally, M. M. (2000). Applied multivariate statistical techniques. Wollongong: FE Publishing. Morrison, D. F. (2005). Multivariate statistical methods (4th ed.). London, Duxbury Advanced Series. Muliak, S. A. (1972). The foundation of factor analysis. New York, N.Y.: McGraw-Hill.

Myers, J. H., \& Mullet, G. M. (2003). Managerial applications of multivariate analysis in marketing. New York, N.Y.: Thomson. Stewart, D. W. (1981). The application and misapplication of factor analysis in marketing research. Journal of Marketing Research, 18(1), 51-62.

Tacq, J. (1997). Multivariate analysis techniques in social science research. Thousand Oaks, CA: Stage Publications. Wichern, D. W. (2001). Applied multivariate statistical analysis. Paramus, N.J.: Prentice-Hall. 